SIMULTANEOUS ESPI MEASUREMENTS USING MULTIPLE WAVELENGTHS AND A COLOR CAMERA

by

\title{
Guillaume Richoz
}

B.A.Sc., College of Engineering and Architecture of Fribourg, Switzerland, 2008

A THESIS SUBMITTED IN PARTIAL FULFILLMENT OF

THE REQUIREMENTS FOR THE DEGREE OF

MASTER OF APPLIED SCIENCE

in

THE FACULTY OF GRADUATE AND POSTDOCTORAL STUDIES

(Mechanical Engineering)

THE UNIVERSITY OF BRITISH COLUMBIA

(Vancouver)

September 2014

(c) Guillaume Richoz, 2014 


\begin{abstract}
Electronic Speckle Pattern Interferometry (ESPI) is typically done using a single monochromatic light source with a monochrome camera. This arrangement enables full-field measurements of a single deformation quantity according to the particular arrangement of the optical system. If a further deformation quantity is to be measured, then the associated optical arrangement must be used sequentially. Here, an alternative approach is described where multiple interferometric measurements are simultaneously made using a color camera imaging monochromatic light sources of different wavelengths. The Red-Green-Blue (RGB) sensors of a conventional Bayer type camera can be read separately, thereby providing three independent color signals and independent ESPI phase maps. An example case is demonstrated here where two Michelson interferometers are combined to form a shearography camera with surface slope sensitivity in two perpendicular directions. By the use of two colors, both surface slopes can be measured simultaneously. ESPI is a relative measuring technique and the third available color is used for absolute phase determination through the Two-Wavelength Method. The availability of the two surface slopes gives the opportunity for the data to be summed numerically to give the surface displacement shape. This application is of significant practical interest because the surface displacement measurement can be made under field conditions by taking advantage of the wellknown stability of shearography measurements.
\end{abstract}




\section{Preface}

All of the work presented henceforth was conducted in the Renewable Resources Laboratory at the University of British Columbia, Point Grey campus.

A version of Chapter 2.2, concerning the color calibration, and Chapter 3.3.1, concerning the MultipleWavelength phase stepping, has been published in Advancement of Optical Methods in Experimental Mechanics, Volume 3: Proceedings of the 2014 Annual Conference on Experimental and Applied Mechanics [Richoz, G. and Schajer, G.S. Simultaneous ESPI Measurements Using Multiple Wavelengths and a Color Camera. ISBN 978-3-319-06985-2]. I was the lead investigator, responsible for all major areas of data collection and analysis, as well as manuscript composition. My supervisor, Dr. Gary Schajer, assisted with some concept formation and made some manuscript edits. Dr. Schajer also developed the algorithms for color separation explained in Chapter 2.2 and for correcting non-uniform shear explained in Chapter 4.3.1.

I was the lead investigator for all the remaining work where I was responsible for concept formation, data collection and analysis, as well as the majority of manuscript composition. 


\section{Table of Contents}

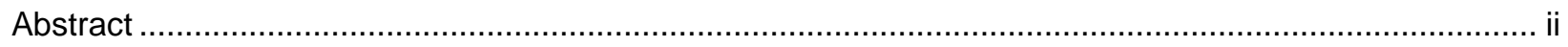

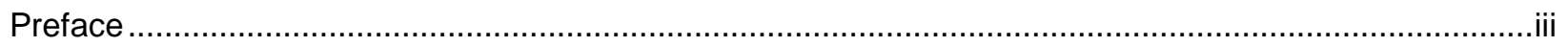

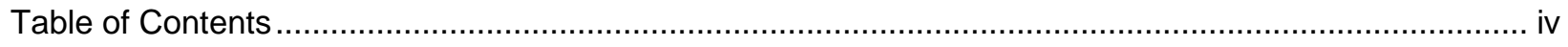

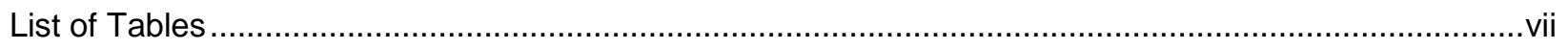

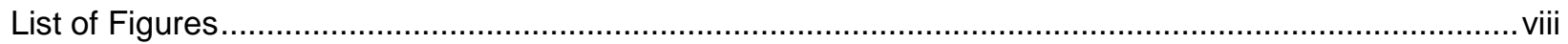

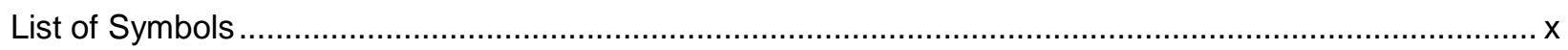

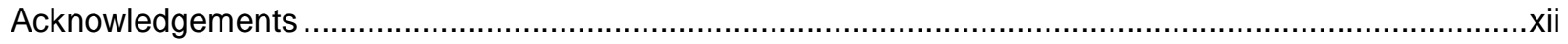

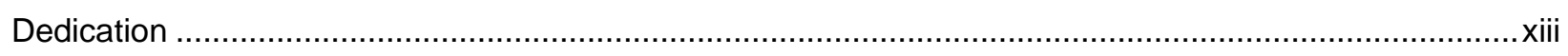

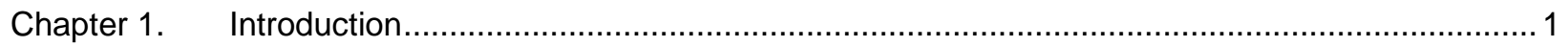

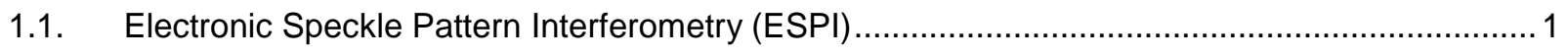

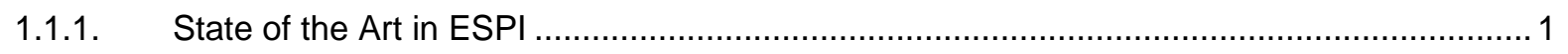

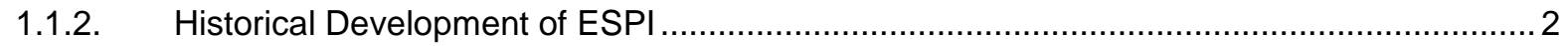

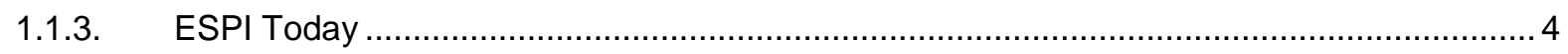

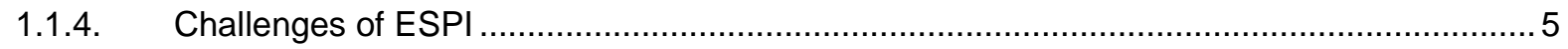

1.2. Multiple-Wavelength Measurements Using a Color Camera .............................................. 6

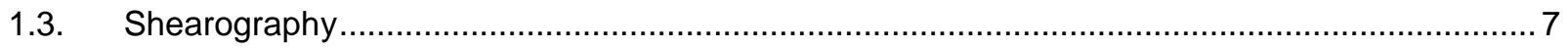

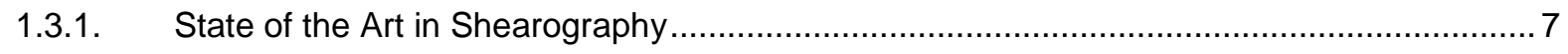

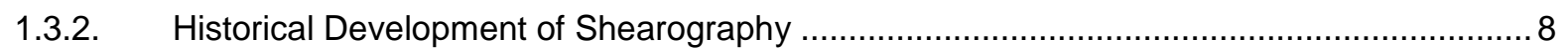

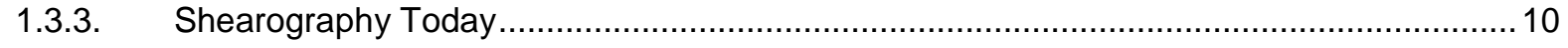

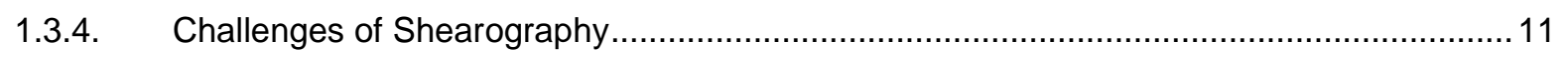

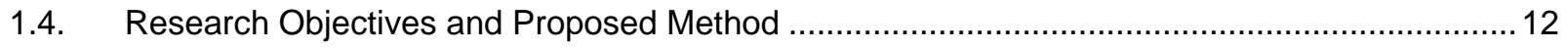

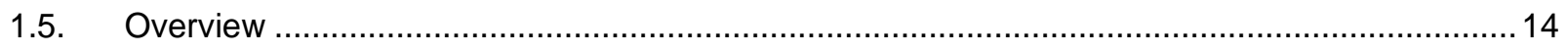

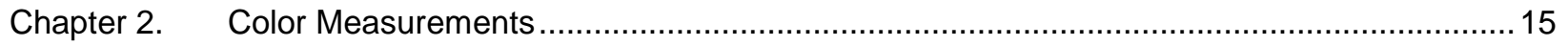

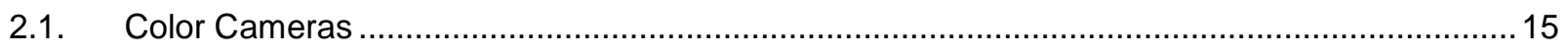

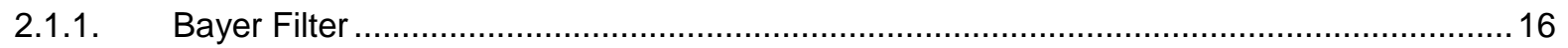

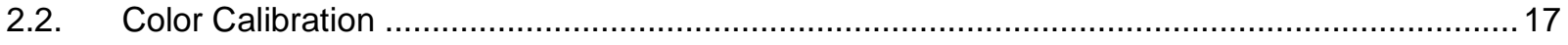

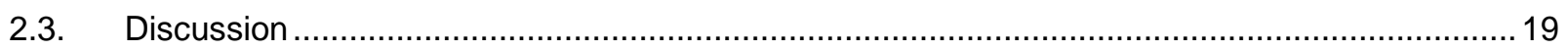




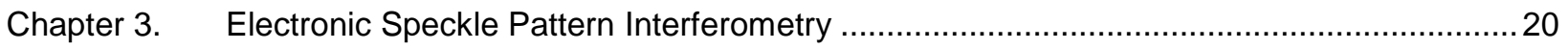

3.1. The Nature of Light and the Laser Speckle Phenomenon ..............................................20

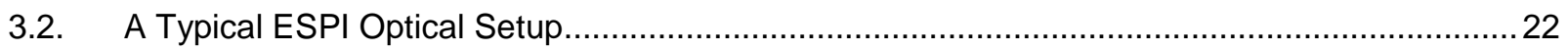

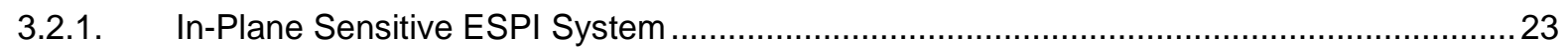

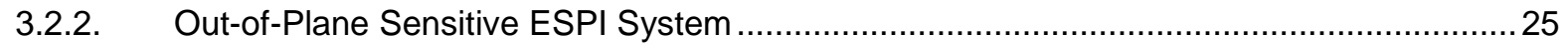

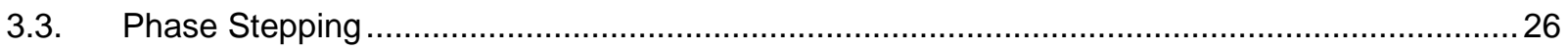

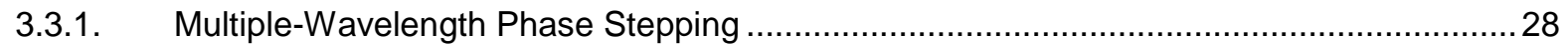

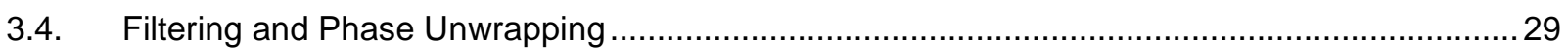

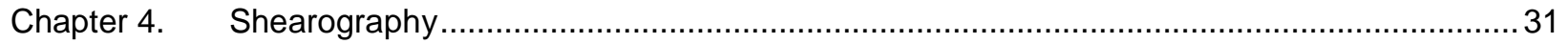

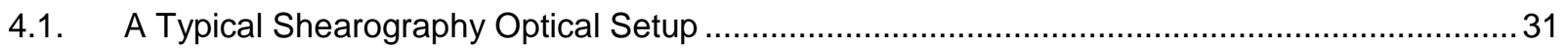

4.1.1. Relationship of Fringes to the Optical Phase Change .................................................. 31

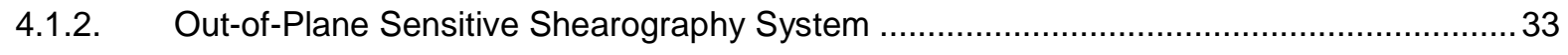

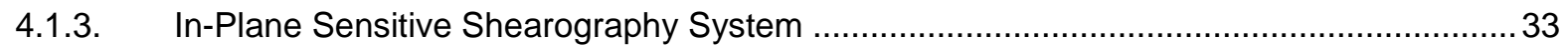

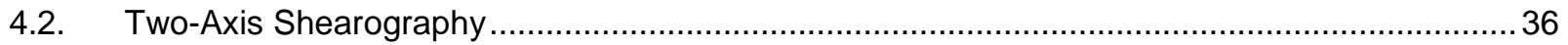

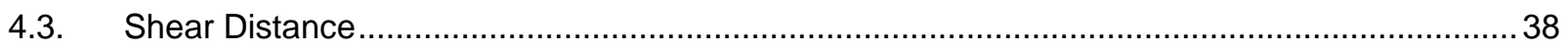

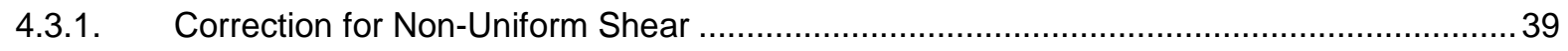

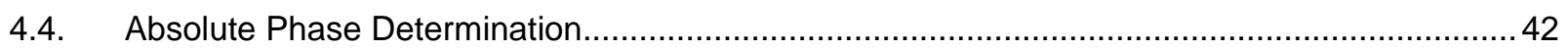

4.5. Surface Slopes Summation and Surface Displacement Shape ............................................ 45

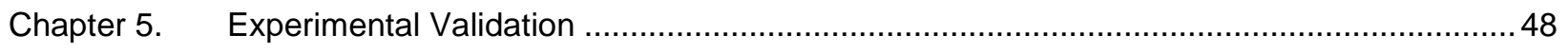

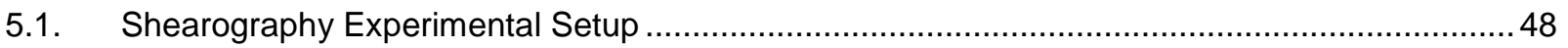

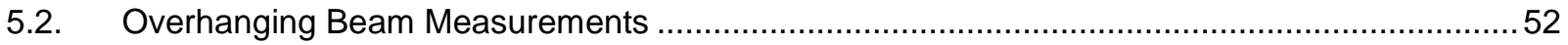

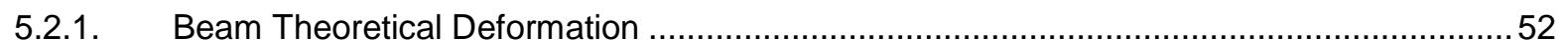

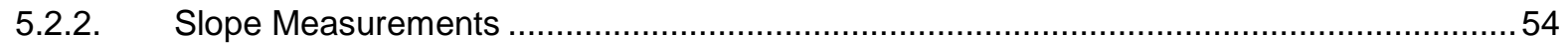

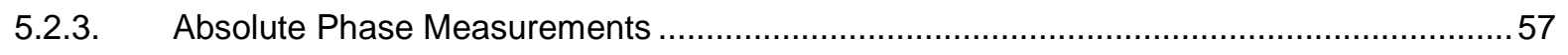

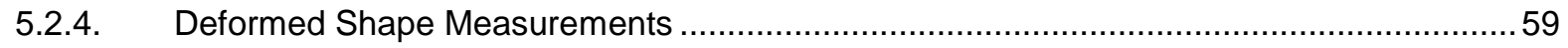

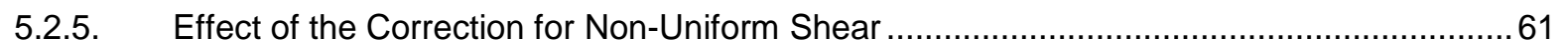

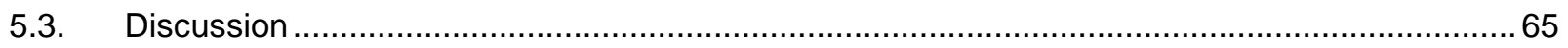

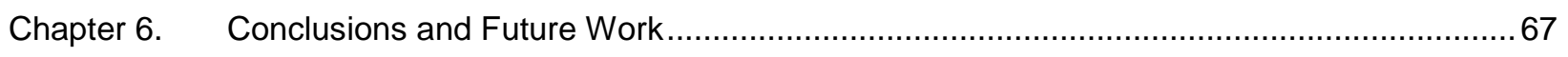

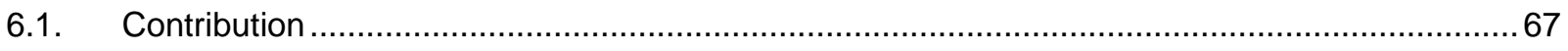




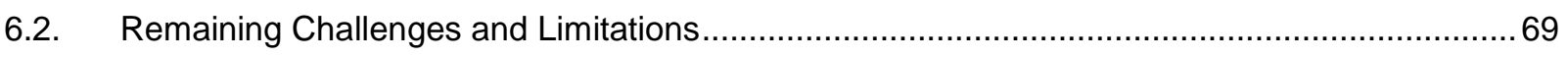

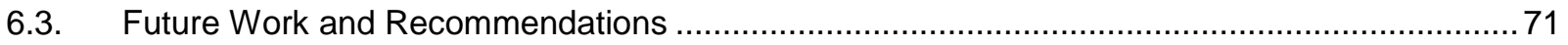

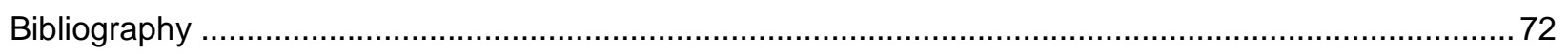




\section{List of Tables}

Table 1 - Slope/strain ratio for a cantilever beam with $2 \mathrm{~mm}$ thickness and 200mm length..................... 36

Table 2 - List of components used for the experimental setup ........................................................ 49

Table 3 - Absolute errors on the relative slope measured with green and blue...................................... 57

Table 4 - Absolute errors on the absolute green phase measured with green and blue. .........................59

Table 5 - Absolute errors on the measured surface displacement shape with green and red. .................60

Table 6 - Absolute errors on the relative slope measured with green and blue.................................... 63

Table 7 - Absolute errors on the absolute green phase measured with green and blue. ........................ 64

Table 8 - Absolute errors on the measured surface displacement shape with green and red. ................65 


\section{List of Figures}

Figure 1 - Laser speckle from a green laser illuminating a rough surface captured with a monochromatic camera.

Figure 2 - Measurement range of different methods for out-of-plane measurement of a concentrically loaded, aluminum circular plate $(D=150 \mathrm{~mm}, t=3 \mathrm{~mm})$, adapted from [6].

Figure 3 - CCDs move photogenerated charge from pixel to pixel and convert it to voltage at an output node. CMOS imagers convert charge to voltage inside each pixel (adapted from Stefano Meroli). 15

Figure 4 - The Bayer arrangement of color filters on the pixel array of an image sensor (Wikipedia)....... 16

Figure 5 - Demosaicing estimates the missing color pixel value by interpolating the neighboring values. 17 Figure 6 - Absolute quantum efficiency graph of the Prosilica GE680C color camera - Allied Vision Technologies. ..... 18

Figure 7 - Geometric representation of the speckle statistics (adapted from [86]). 20

Figure 8 - Physical origin of speckle for (a) free-space propagation, (b) an imaging system (adapted from [87])

Figure 9 - Change in the optical path length due to surface deformation. ...........................................22

Figure 10 - Optical configuration of an in-plane ESPI system. ....................................................... 23

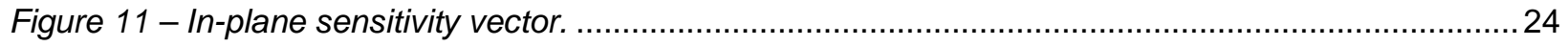

Figure 12 - Optical configuration of an out-of-plane ESPI system. ...................................................... 25

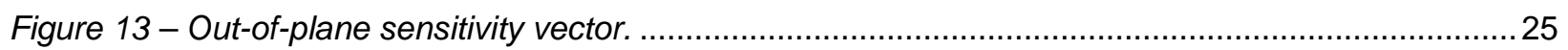

Figure 14 - Sinusoidal intensity profile of a single pixel before and after deformation. ...........................22

Figure 15 - (a) fringe pattern (b) sawtooth wrapped phase distribution. ............................................. 30

Figure 16 - (a) unwrapped phase map (b) profile of the unwrapped phase distribution. .......................... 30

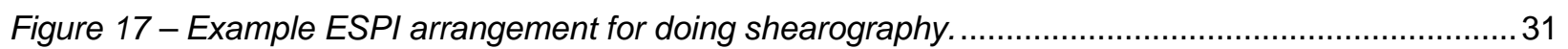

Figure 18 - Combination of deformation (a) out-of-plane deformation (b) in-plane deformation. ...............32

Figure 19 - Optical configuration of an out-of-plane shearographic system. …................................ 33

Figure 20 - Optical configuration of an in-plane shearographic system................................................. 34

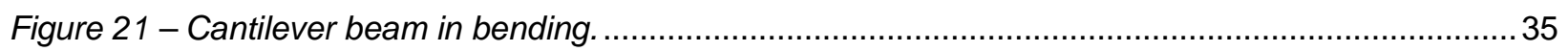

Figure 22 - Optical configuration of a two-axis shearographic system. ................................................ 36

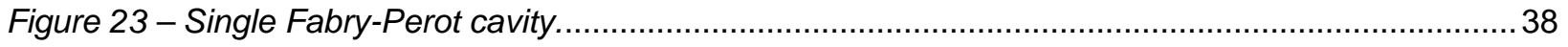

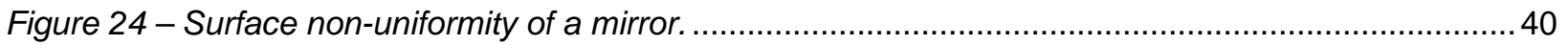

Figure 25 - Shear maps (a) $x$-shear map in $x$-direction (b) $x$-shear map in $y$-direction (c) $y$-shear map in $x$ -

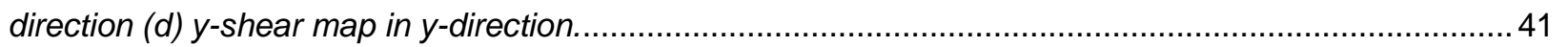

Figure 26 - Wrapped and unwrapped phases of a circular membrane loaded at its center. ....................43

Figure 27 - Change in optical path length due to surface rotation (a) reference state (b) deformed state.

Figure $28-$ One direction slope summation.

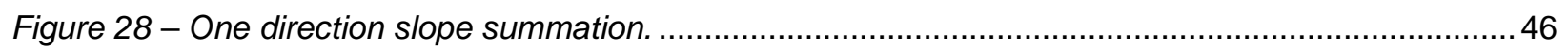


Figure 29 - Schematic of the summation method to retrieve the displacement field.

Figure 30 - Experimental setup for doing Two-Axis Shearography to measure surface deformations on an overhanging beam in bending.

Figure 31 - RGB laser source (a) global view (b) dichroic filters for beam combination. 50

Figure 32 - Overhanging beam test system (a) global view (b) region of interest with red light only (c) modified micrometer. .

Figure 33 - Two-axis shearography interferometer (a) interferometer close up, (b) interferometer with imaging system. 52

Figure 34 - Overhanging beam (a) plan view (b) side view. .52

Figure 35 - Overhanging beam out-of-plane displacement for an 8mm displacement on the micrometer.

Figure 36 - Slope difference measurements in x direction using a single-axis interferometer (a) wrapped phase map blue laser, (b) wrapped phase map red laser, (c) slope difference results. .55 Figure 37 - Slope difference measurements with a two-axis interferometer (a) wrapped phase map blue laser $=\partial w / \partial x,(b)$ wrapped phase map green laser $=\partial w / \partial x,(c)$ wrapped phase map red laser $=\partial w / \partial y$ with filtering, (d) slope difference results for blue laser, (e) slope difference results for green laser. ................56 Figure 38 - Relative slope of blue and green laser for an 8mm imposed displacement........................5 57 Figure 39 - Absolute phase determination for (a) $4 \mathrm{~mm}$ and (b) $8 \mathrm{~mm}$ imposed displacement...................58 Figure 40 - Reconstructed surface displacement shape (a) orthogonal view for a $4 \mathrm{~mm}$ imposed displacement, (b) bottom view for $4 \mathrm{~mm}$, (c) longitudinal shape averaged across the width. 60 Figure 41 - Slope difference measurements with a two-axis interferometer (a) uncorrected wrapped phase map blue laser $=\partial w / \partial x$, (b) corrected wrapped phase map blue laser, (c) uncorrected wrapped phase map green laser $=\partial w / \partial x,(d)$ corrected wrapped phase map green laser, (e) uncorrected wrapped phase map red laser $=\partial w / \partial y$, (f) corrected wrapped phase map red laser.

Figure 42 - Slope difference measurements with a two-axis interferometer (a) slope difference results for

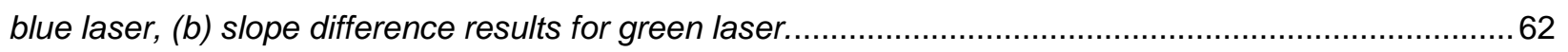
Figure 43 - Relative slope of blue and green laser for an 8mm imposed displacement......................... 63 Figure 44 - Absolute phase determination for (a) 4mm and (b) 8mm imposed displacement..................64 Figure 45 - Wrapped reconstructed phase maps for surface displacement shape reconstruction for $4 \mathrm{~mm}$ imposed displacement with correction for non-uniform shear (a) wrapped phase map without mirror misalignment (b) wrapped phase map with mirror misalignment. 65 


\section{List of Symbols}

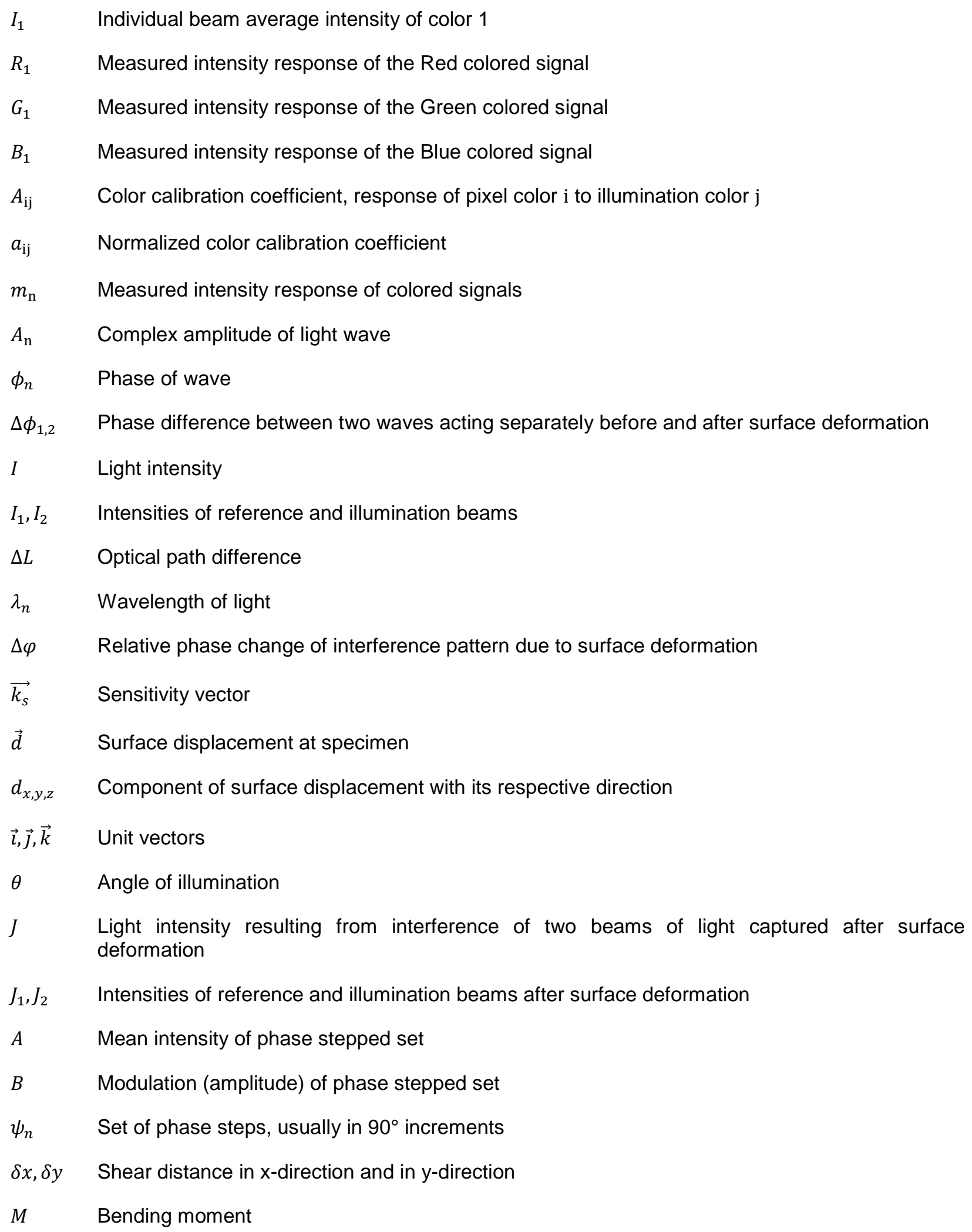




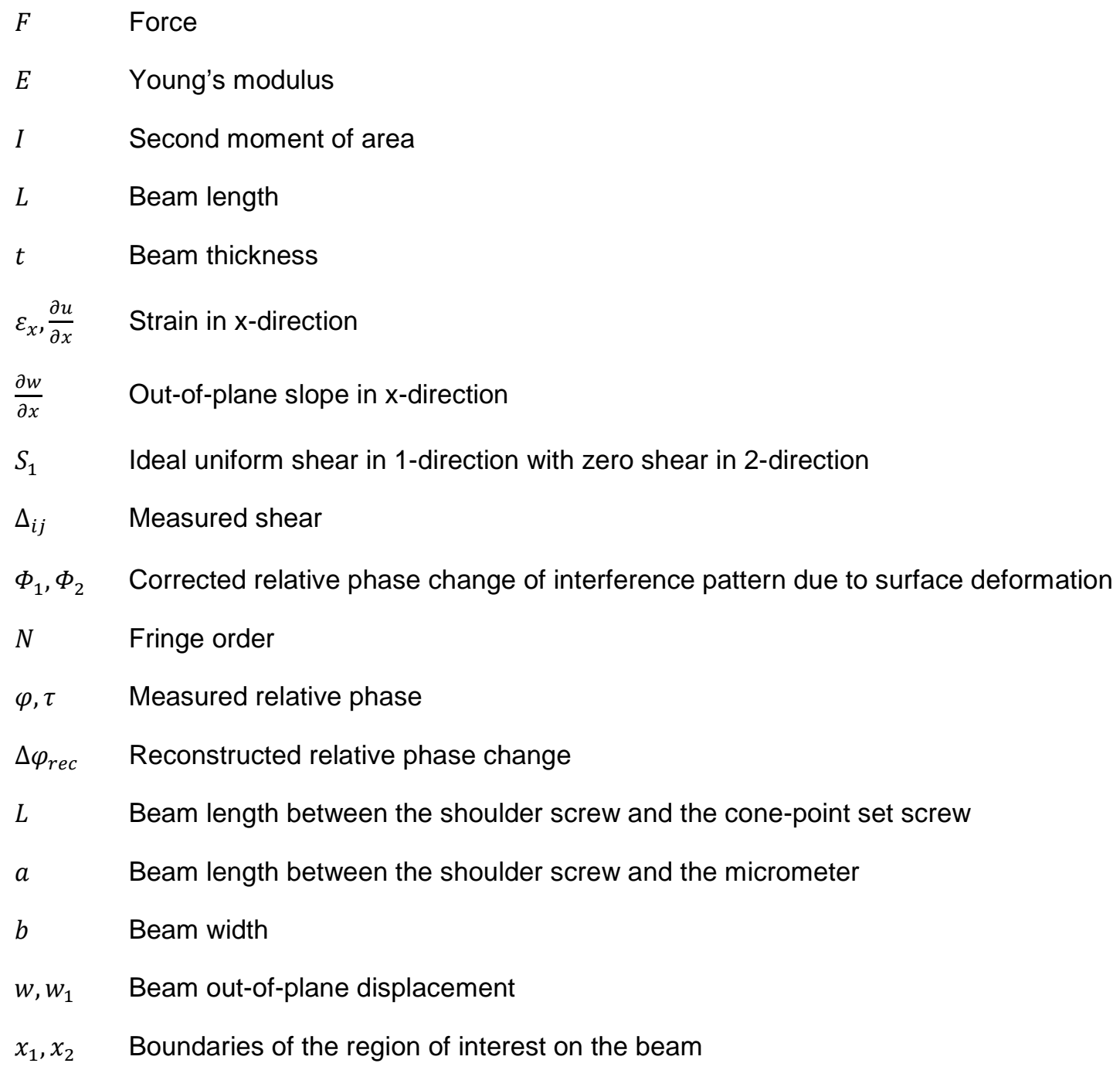




\section{Acknowledgements}

First and foremost I would like to express my warmest gratitude to my supervisor, Dr. Gary Schajer, for his guidance, support, and inspiration during this work. Professor Schajer is one of the smartest people I have had the chance to meet. His ability to explain complex ideas is second to none. One simply could not wish for a better supervisor. Thank you.

I would like to thank Markus Fengler and his team, in the Machine Shop in the department of Mechanical Engineering, who gave me precious support throughout the duration of this research. I also would like to thank Glenn Jolly and his team, in the Electronics Shop. This accomplishment would not have been possible without them.

Furthermore, I would like to thank my lab mates from the Renewable Resources Laboratory, Darren Sutton, Joshua Harrington, Samuel Melamed, Ted Angus, Wade Gubbels, and Yuntao An for the many useful suggestions, ideas, and most importantly for their friendship.

Last but not the least, I would like to thank my family for their love, patience, and unfailing support throughout my life. I would not have come this far without their help. 


\section{Dedication}

To my Family 


\section{Chapter 1. Introduction}

\subsection{Electronic Speckle Pattern Interferometry (ESPI)}

\subsubsection{State of the Art in ESPI}

The Nobel Prize in Physics 1971 was awarded to Dennis Gabor [1] for his invention and development of the holographic method. Dr. Gabor said during a conference: "The novelty in holography is speckle noise. This has really nothing to do with holography; it is a consequence of coherence. It has a special standing among noise phenomena because it is not really noise but unwanted information. When we put a sheet of white paper into laser light, the reflected light conveys to us information on the roughness of the paper in which we are not at all interested. I am glad that at last Dr. J. M. Burch [2] has shown that we can make some use of this information."

These random granular patterns, called speckle patterns and which create primarily unwanted information or noise, occur when an optically rough surface is illuminated with coherent light. When a surface, such as a piece of paper, a concrete wall, or a metallic surface, is illuminated by a laser beam and is viewed by someone, a speckle pattern, as illustrated in Figure 1, is formed on his retina. Because speckle patterns carry information about the object's surface, if the observer moves his head, it will result a new speckle pattern. This phenomenon, which is used in three main speckle techniques such as speckle photography, speckle interferometry and speckle shear interferometry, results from many scattered waves, each having a random relative phase, interfering constructively or destructively.

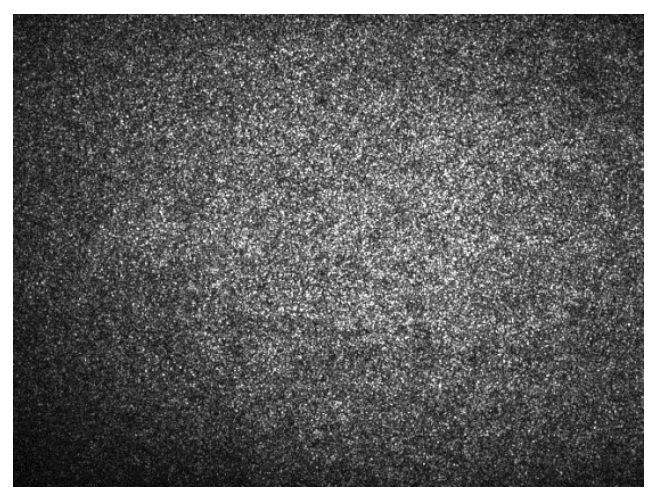

Figure 1 - Laser speckle from a green laser illuminating a rough surface captured with a monochromatic camera.

Speckle Interferometry ( $\mathrm{SI}$ ) is a well-known successor of Holographic Interferometry $(\mathrm{HI})$. Holographic interferometry differs from speckle interferometry by the way in which the fringe pattern is formed. In traditional holographic interferometry usually a photosensitive material, such as a high-resolution photographic film, is used to record interference of two wavefronts. This interference emanates from a 
reference beam of light, which is shone directly incident to the recording instrument, and a beam that has been scattered off an object. In speckle interferometry the fringe pattern arises by electronic processing of two interference patterns recorded on a low resolution medium such a CCD.

Speckle photography and speckle interferometry both involve photography and both involve interference. In 1975 Karl A. Stetson suggested [3] that if there are regions in the two images where the speckle patterns of each are well correlated, let the process be referred to as speckle photography. If, instead, the fringes form as a result of fluctuations in the correlation of the speckle patterns between two images, whether or not there is translation between the correlated portions of the patterns, let the process be called speckle interferometry.

Electronic Speckle Pattern Interferometry (ESPI) is an imaging technique that uses coherent laser light to visualize and quantify small surface displacements of an object [4,5]. The technique has been applied to the solution of a wide range of problems in strain/stress and vibrational analysis, Non-Destructive Testing (NDT), quality control, design validation and optimization, and in fluid flow visualization [6-8]. With modern data processing systems, a large amount of experimental data can be processed and displayed effectively in real time.

ESPI is a very versatile method for measuring surface deformations and many different deformation quantities can be measured depending on the optical configuration used. Typical measured quantities are in-plane displacements, out-of-plane displacements, out-of-plane slopes and in-plane strains. In all these cases a separate optical configuration is needed for each measurement; if further measurements are required then further optical systems are needed.

\subsubsection{Historical Development of ESPI}

The EPSI technique, which has been developed from the holographic and speckle techniques, was first demonstrated in 1971 by Butters and Leendertz at Loughborough University of Technology and simultaneously by Macovski et al at Stanford Research Institute [9]. The basic principle of ESPI was to combine speckle interferometry with electronic detection and processing to allow real-time measurement of the object. The development of this novel technique has enabled the advantages of optical inspection to be combined with equipment that overcomes some traditional problems of holography, such as the high time-consuming need for film processing. The visual information or photons are converted into electrical current and then processed electronically. This technique allows a multitude of pre-processing to be applied to the recorded speckle patterns. A variety of complicated algorithms can be implemented to improve the fringe quality. Current computer and programming technology enable manipulation of several images in quasi real-time at relatively low costs. The acronym DSPI for Digital Speckle Pattern 
Interferometry can also be found in the literature because of the use of both digital images and processing techniques but the name ESPI still remains the most popular internationally.

The first results recorded by Butters and Leendertz with a vidicon TV camera having 180x350 pixels were not satisfying mostly because of the low detector resolution, low sensitivity, and high signal-to-noise ratio. The ESPI system was applied to measure vibrations of a disc and torsion of a square metal plate. Butters showed the early progress of speckle pattern interferometry using television processing with an arrangement for inspecting turbine blades with a resolution of 0.0005 inch [10]. In 1975, A.E. Ennos described the difference between ESPI and holographic interferometry [11], he also summarized the work achieved by Butters and Leendertz on the analysis of vibrations and displacement measurements.

Till the mid-80s, the development of the ESPI technique has been essentially conducted by three research groups, led by Butters at Loughborough University, by Løkberg at the Norwegian Institute of Technology in Trondheim, and by Nakadate at Tokyo Polytechnic University. Butters improved the holographic two-wavelength contouring method by using ESPI and an argon laser [12]. His team incorporated a pulsed ruby laser into an electronic speckle pattern interferometer [13]. With the ruby laser set to double pulse operation the principle of speckle pattern addition has been shown to produce high contrast fringes when observing vibrational and transient events. Løkberg worked on the mapping of the contours of constant phases across sinusoidally vibrating objects using reference wave phase modulation in time-average ESPI [14]. A new technique using a speckle reference beam was demonstrated by Slettemoen [15]. The Norwegian team followed the evolution of laser technology and integrated it into the ESPI technique. A continuous wave laser was used to measure induced vibrations of very unstable objects [16]. Vibration measurements on the human ear drum in vivo have been demonstrated with the use of a continuous wave laser [17]. Continuing on measuring movements inside a biological body, Løkberg combined the properties of ESPI with the flexibility of fiber-optic illumination and observation [18]. Infra-red lasers, like the CO2 laser, have been used to obtain fringe patterns of vibrating objects [19]. In 1988, Løkberg measured vibrations for a total path-length difference of up to $200 \mathrm{~m}$ with the object being outdoors in bright sunshine [20]. Nakadate used digital image processing techniques, such as subtraction, summation, and level slicing [21]. These digital techniques allowed to obtain easily high contrast fringes and eased restriction on the speckle size and the laser power. The Japanese team reported a computer aided speckle interferometry system in 1983 [22]. Two years later, they introduced a fringe scanning method with phase-shifted speckle interferograms, which made possible to evaluate automatically speckle interferograms to give numerical data of the deformation [23].

K. Creath was the first to use a Reticon diode array camera in an ESPI system [24]. She was also the first to introduce a phase shifting technique in speckle interferometry to obtain quantitative data of the object displacement [25]. She also investigated the use of a diode laser source [26]. 
D. Kerr et al. applied a Fourier filtration algorithm to speckled fringe patterns, obtained with an electronic speckle pattern interferometer [27]. Moore et al. established a technique suitable for the simultaneous measurement of two mutually orthogonal in-plane displacement fields which is useful in the field of planestrain displacement analysis [28]. M. Owner-Petersen investigated the effects of decorrelation on the correlation fringe pattern observed in speckle-pattern interferometers [29]. H. Saldner et al. presented an optical configuration for simultaneous measurement of out-of-plane displacement and slope change from a single configuration [30]. G. Pedrinia et al. used a double pulse electronic speckle pattern interferometer for vibration measurements with a CCD camera [31]. By operating the ruby laser at higher energy it was possible to record interferograms of larger object e.g. $1 \times 1 \mathrm{~m}^{2}$. Van Haasteren and Frankena developed a multi-camera phase-stepping speckle interferometer that generates three phase-stepped interference patterns simultaneously for real-time displacement measurement [32]. Chung Ki Hong et al. developed a least-squares fitting of the phase map to remove noise from the deformation phase map obtained by a phase-shifting electronic speckle pattern interferometry [33].

After 1995, the ESPI technique has been widely democratized and developed for all kind of application fields. Franze et al. proposed a temporal phase evaluation for measuring large-object deformation [34]. Schajer and Steinzig proposed a mathematical method for calculating residual stresses from hole drilling ESPI data, independent of rigid-body motions associated with hole drilling or temperature changes [35].

The effort on the digital processing has also been pushed forward. Aebischer and Waldner described an anisotropic sine/cosine average filter for filtering speckle-interferometric phase fringe patterns [36]. Burton et al. proposed a novel technique that uses a fan two-dimensional continuous wavelet transform to phase demodulate fringe patterns [37]. Qian Kemao implemented a two-dimensional windowed Fourier transform for fringe pattern analysis [38].

\subsubsection{ESPI Today}

Electronic speckle pattern interferometry has become a very successful method with the ever increasing development of digital image recording and processing. The ESPI method measures very small surface displacements with the advantages of being a non-contact, nondestructive, and whole field measurement technique. It also offers other benefits such as rapidity, real-time measurements can be achieved, and it provides extremely accurate measurements in the order of a wavelength of light.

ESPI has the potential to be used for vibration monitoring, deformation-displacement visualization, contouring, surface roughness measurement, or strain/stress analysis. ESPI technique combined with stroboscopic laser illumination can be used to measure 3D harmonic vibration on any components and structures. Huang and Peng worked on improving the design of rotor blades with the help of ESPI [39]. 
Analysis of thermomechanical deformations in thermoelectric generators were performed using electronic speckle pattern interferometry [40], where temperature gradients as well as small surface deflections in the $\mu \mathrm{m}$ range had to be measured simultaneously. Koch et al. used ESPI for contouring surfaces with discontinuities [41] to make the technique more suitable for industrial applications where typical surfaces often show discontinuities, like steps or holes. Hertwig from the Swiss Federal Institute of Technology of Zurich performed a set of surface roughness measurements on surfaces having an average mean roughness $\mathrm{R}_{\mathrm{a}}$ lying between $12.5 \mu \mathrm{m}$ and 1.6 $\mu \mathrm{m}$ [42]. Yang et al. measured microscopic deformations for high-resolution determination of 3D strain distributions on mouse femora [43]. They built an advanced ESPI system and integrated local contour information to deformation data.

The company Dantec Dynamics has developed a 3D-ESPI system, which is designed for complete three dimensional, full-field and highly sensitive displacement, strain and stress analysis. It delivers Young's modulus and Poisson's ration. Measurement can be performed on any material, also on anisotropic material. The measuring sensitivity goes from 0.03 to $1 \mu \mathrm{m}$ for a measuring area up to $200 \times 300 \mathrm{~mm}^{2}$. The American Stress Technologies company sells hole-drilling equipment for residual stress testing. Their "Prism" instrumentation measures changes in the part surface resulting from hole drilling and determines the previously existing residual stresses. The company Optonor AS offers has industrialized an ESPI system, VibroMap 1000, for vibration and static displacement measurements. Their product provides animations and real time functions.

\subsubsection{Challenges of ESPI}

The application in industry of the electronic speckle pattern interferometry technique is limited by the effects of rigid-body motions during ESPI measurements. Speckle patterns carry information about the object's surface and their intensity is measured pixel by pixel. This procedure is carried out before and after deformation of the test specimen to measure the relative optical phase change induced by the deformation. To be effective for the measurement, speckles need to be approximately the same size as a pixel. When large rigid-body motions are involved during measurement, larger than the pixel size of the digital camera used for recording speckle patterns, decorrelation of speckles appears. This decorrelation effect will simply corrupt the ESPI measurement.

The ESPI technique is very sensitive to environmental perturbations such as vibrations or temperature changes around the test specimen. A basic ESPI setup consists in one coherent beam of light being split in two parts which are brought back together to interfere between each other on the test specimen or the camera sensor. When temperature changes, air currents, humidity, pressure variations, or vibrations affect one of the split beam more than the other one, the measured phase is affected and fringe drift 
occurs. One fringe, dark or bright, on the image corresponds to surface displacements of the object by a half wavelength of the light source used.

ESPI is a relative measuring technique. A major problem in quantitative ESPI measurement is the determination of the absolute phase. ESPI commonly uses phase stepping for retrieving the relative phase change created by the object deformation. This relative phase change is proportional to the surface deformations. The phase stepping consists in two sets of four phase-stepped images taken before and after deformation. An arctangent function is used to calculate the relative phase, which yields a phase map with modulo $2 \pi$ repetitions. To remove the $2 \pi$ ambiguities, phase unwrapping is applied and creates a continuous phase map. However, getting the absolute phase change is practically unreachable without knowing the boundary conditions.

\subsection{Multiple-Wavelength Measurements Using a Color Camera}

The concept of using multiple-wavelength for optical measurement techniques has been first developed for holography. In 1967, Hildebrand and Haines applied multiple-wavelength and multiple-source holography to contour generation [44]. They described the two methods for obtaining three-dimensional images with constant-range contours superimposed on them. When the source that illuminates the object is changed in position or frequency during the exposure, or when two sources or frequencies are used simultaneously, then the hologram contains more object information than in the single-source or frequency case. At that time, holograms were recorded onto a film very similar to photographic film and cameras were not involved.

The first CCD or Charge Coupled Device has been invented in 1969 by George Smith and Willard Boyle. Color recording with a CCD camera depends on the color filter placed over the pixel sensors, not the sensor itself. Therefore, there was never a first "color CCD camera", but the first color filter array was invented by Dr. Bayer from Eastman Kodak in 1976 [45].

Multiple-wavelength measurements allow to get simultaneously more information than with in a singlesource arrangement. Multiple-measurement ESPI experimental setups have already been studied [6,46], but both setups involved black and white CCD camera. These involve using multiple optical configurations that are activated sequentially to measure each desired quantity separately. This approach works well for measurement of static deformations, but is not well suited to dynamic measurements. In addition, the measurements are typically made using several separate cameras, and these must be carefully aligned to ensure they record consistent measurement areas.

The use of a color camera allows simultaneous and spatially aligned measurements of multiple independent signals, one for each measured color. This is possible because the sensor of a color 
camera contains interlaced sets of color sensitive pixels, Red, Green, and Blue (RGB) in the conventional Bayer arrangement [45]. Each of the colored pixels can be read separately, thus allowing independent and simultaneous measurement of three different colored speckle images. Such simultaneous measurement is particularly valuable for surface vibration measurements. Techniques exist for making ESPI measurements from a single optical image [47], thereby allowing high-speed sequential acquisition of ESPI data. Now, three independent measurements can be made simultaneously, so automatically making them coordinated in time. In addition, the three speckle images are mutually aligned on the Bayer sensor, so also automatically making them coordinated in space. All of these features extend the range of use of ESPI measurements and simplify optical design.

\subsection{Shearography}

\subsubsection{State of the Art in Shearography}

Shearography was first known under the name of speckle shear interferometry. ESPI is a very flexible technique and it can be used to measure many different deformation quantities, depending on the optical geometry used. As in ESPI, the originally unwanted speckle phenomenon is used in shearography because speckle patterns carry information about the object's surface. As shown in Figure 2, the measurement range for out-of-plane measurement of a concentrically loaded plate is larger for shearography.

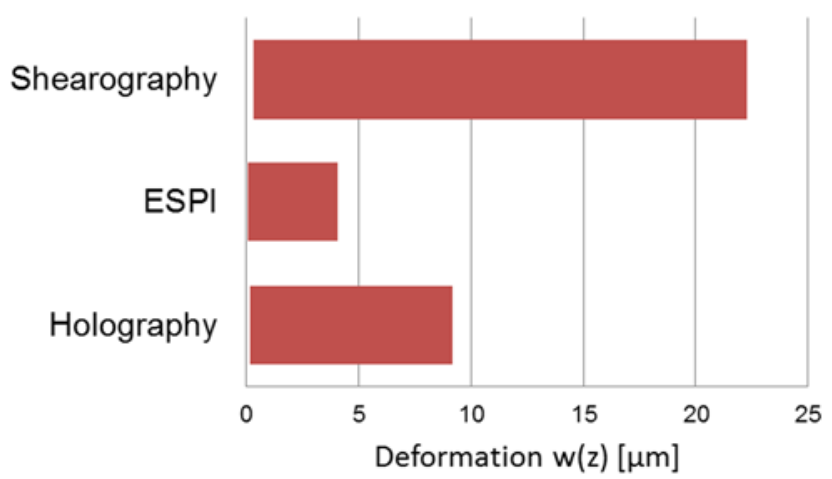

Figure 2 - Measurement range of different methods for out-of-plane measurement of a concentrically loaded, aluminum circular plate $(D=150 \mathrm{~mm}, t=3 \mathrm{~mm})$, adapted from [6].

In 1973 Leendertz and Butters were the first to measure bending moments on a square metal plate using a shearing interferometer similar to the Michelson interferometer [48]. The Michelson interferometer is an amplitude splitting interferometer where a partial reflector divides the amplitude of the incoming wave into 
two separate beams which are then recombined. Having one of its mirror intentionally tilted at a small angle, the shearographic interferometer produces a pair of laterally sheared images on the image plane. These two sheared images interfere each other thus producing a speckle pattern on the recording media. Speckle patterns, before and after deformation, are recorded to produce by comparison a fringe pattern representing the first derivative of the out-of-plane deformation of the specimen surface. The out-of-plane displacement needs to be differentiated twice to yield the curvature. By using shearography, the first differentiation is performed optically then the bending moments can be calculated from the fringe spacing, which represents the radius of curvature.

Shearography is an important diagnostic tool used essentially for qualitative inspection, and it is very popular in the field of nondestructive testing. Shearography is applied to different kind of visual inspections such as: quality inspection of internal-layers defects in aircraft and vehicle tires; flow visualization of composite panels, pressure vessels, pipelines, and microelectronic packaging; inspection of fiber reinforced polymer concrete.

Electronic Speckle Pattern Shearing Interferometry for ESPSI has been used in the literature to depict the use of a digital recording device. Shearography still remains the most popular name to describe the speckle shear interferometry. Now CCD cameras are replacing holographic plates thus removing the need for the time consuming wet processing. Once the signal being digitalized, it is possible to measure the phase distribution across the image to make quantitative measurements. Several techniques have been developed to retrieve the phase, such as the phase stepping technique which consists in recording a set of images with a known phase step between them. The recorded images are processed to produce a wrapped phase map with a phase modulo $2 \pi$, which needs then to be unwrapped through a process known as phase unwrapping. The resulting phase is proportional to the first derivative of the out-of-plane deformation depending on the geometrical configuration of the interferometer.

Shearography overcomes several limitations of holography. To get strain with holography it is necessary to numerically differentiate the measured displacement. Shearography measures directly surface slopes or strain according to the setup configuration. The need for a reference beam, as in holography and ESPI, is eliminated. This leads to simpler optical setup with an almost-common path configuration offering to the technique the advantage of having a low sensitivity for common mode disturbances such as vibrations or air turbulence. Another advantage is the possibility of using lasers with reduced coherence length. Shearography does not require special vibration isolation and can be applied for onsite measurements.

\subsubsection{Historical Development of Shearography}

Shearography can be found in the literature under several names, such as speckle shear interferometry, 
speckle shearing interferometry, or speckle pattern shearing interferometry. This optical technique, member of the speckle interferometry family, is born in the hands of Leendertz and Butters in 1973.

The term shearography has been used the very first time in 1981 by Y.Y. Hung in a publication relating shearography as a new optical method for nondestructive evaluation of tires [49]. Michael Y.Y. Hung and his research group are still the most active in the development of Shearography. Professor Hung founded the Croucher Optical Non-destructive Testing and Quality Inspection (CNDT) Laboratory in City University of Hong Kong in 2003. To give a brief overview of his work, some of his publications will be summarized here. In 1974 Hung and his team measured the derivative of out-of-plane displacement of a circular plate clamped along its boundary and centrally loaded [50]. The fringe patterns were obtained by the doubleexposure technique. The same year Hung and Taylor presented a technique to measure displacement derivatives by using a camera having a lens with two apertures and equipped with a shearing mechanism [51]. Hung et al. presented a new speckle-shearing interferometric method that enables spatial derivatives of surface displacements in four different directions to be determined simultaneously [52]. With Liang, Hung described a new image-shearing camera which focuses two laterally sheared images at the film plane for direct measurement of out-of-place displacement derivatives [53]. With Durelli, he used a multiple image-shearing interferometric camera to measure the derivatives of surface displacements with respect to three directions simultaneously [54]. In 1982, Hung compared shearography with holography and other nondestructive techniques [55]. Takezaki and Hung developed a technique which enables shearography to measure second derivatives of deflection directly with the use of fictitious carrier fringes in the form of periodic linear fringes of uniform spacing [56]. Hung et al. presented a technique, based on generating a carrier fringe pattern, which eliminates the ambiguity in shearographic fringe interpretation [57]. In 2000 Hung et al. reviewed three shearographic methods for surface profile measurement, namely, the double-wavelength method, the double-refractive method, and the two point source method [58].

The evolution of experimental setups described in the literature represents well the progress of the shearography technique. Back in 1974 Denby and Leendertz described in-plane strain measurements with the novel use of television equipment [59]. One year later, Hariharan presented a shearing interferometer consisting of two identical diffraction gratings attached directly to the lens mount of the camera used to record images of the test object [60]. Debrus developed a speckle shearing interferometer with a Savart plate, supposedly less sensitive to vibrations than a Michelson interferometer used so far, so as to obtain derivatives of the out-of-plane displacement [61]. Betser et al. recorded slope and curvature contours of flexed plates using a grating shearing interferometer [62]. In 1980, Nakadate et al. measured spatial derivatives of surface displacement and modal vibration amplitude of objects with an image-shearing camera using a Fresnel biprism of small angles and a digital TV-image processing facility [63]. The next year, Taylor et al. showed that a common glass plate can be an adaptable and effective tool for use in speckle-shearing interferometry [64]. Sirohi et al. developed a method which provides a 
linear shear in the image plane and they conceived the split-lens speckle shear interferometer which consists in a well-corrected achromat cut into two equal parts [65]. Sirohi, Mohanty, and Joenathan presented a new speckle-shear technique that gives a $\theta$-shear with double dove prisms [66]. Sharma, Sirohi, and Kothiyal measured simultaneously slope and curvature with a three-aperture speckle shearing interferometer [67]. In 1988, Ganesan et al. presented an universal speckle shear interferometer where lateral, radial, rotational, and inversion shear can be obtained [68]. Kadono et al. studied experimentally the use of a liquid-crystal cell employed as a phase shifter to implement the phase-shifting method for the conventional speckle-shearing interferometer [69]. In 1997, Aebischer, and Waldner presented a simple optical set-up with three light sources arranged in a particular symmetry and an associated procedure which allows accurate isolation of all six displacement derivatives [70]. Sennhauser et al. developed a multi-wavelength speckle pattern shearing interferometer for the determination of two-dimensional strain distributions [71].

The progress of shearography also opened the doors of new applications for the technique. In 1987, Anastasi et al. used shearography to nondestructively investigate simulated delaminations in a composite laminated plate [72]. In 1991, Toh et al. showed that time-average shearography can be used successfully to detect debonds or delaminations in composite plates and that the technique gives a good estimation of the flaw sizes [73]. Shakher and Nirala used shearography for measuring the temperature profile inside a gaseous flame [74].

\subsubsection{Shearography Today}

As holographic interferometry and ESPI, shearography has the advantages of being a non-contact, nondestructive, and whole field measurement technique. In a strain/stress analysis point of view, shearogaphy has the advantage over both holography and ESPI of directly measuring derivatives of surface displacements. Additionally, shearogaphy systems have a common optical path between the object and the shearographic interferometer as well as between the illumination source and the object under test. Furthermore, most shearing devices offer an almost-common optical path between the interferometer and the sensor plane, and thus lead to very stable measurements even under harsh environmental conditions. These advantages have made shearography a well-accepted tool for qualitative nondestructive testing with a wide range of applications including stress/strain analysis, as well as vibration studies. Shearography is widely used in the rubber industry for tires inspection and also in the aerospace industry for nondestructive testing of aircraft structures made of composite.

Shearography has been demonstrated to be an effective tool for vibration monitoring, deformationdisplacement visualization, contouring, or strain/stress analysis. Shearography offers the same range of applications than ESPI with some extra and non-negligible advantages as cited above. Bhaduri et al. 
used a dual-function shearography system for the visualization of resonance frequencies and their mode shapes [75]. In 2006, Habib measured thermal expansion coefficients of thin film of different organic coatings by using shearography [76]. Fernández et al. proposed a novel approach to surface contouring using two-source phase-stepping digital shearography where the illumination source is displaced in a prescribed manner to produce a phase difference increment [77]. Sanati et al. determined the residual stresses in parallel tubular channel angular pressing processed tubes using an incremental blind hole drilling with digital shearography instead of conventional hole-drilling method of strain gauges [78]. The main field of application for shearography still remains in nondestructive testing. Kurpka et al. presented recent installations of automatic inspection systems in aerospace industries where for example complete rotor blades are inspected within 10 minutes on delamination and debonding of the composite structure [79]. Růžek et al. compared ultrasonic C-Scan with shearography for impact defects identification on sandwich panels [80], and concluded that shearography is able to detect Barely Visible Impact Damages (BVID) very well opposingly to the ultrasonic technique.

The company Dantec Dynamics has developed a fully portable and compact shearography system, Q800. The system consists of a miniaturized shearographic sensor with integrated high-resolution CCD, 1392x1040 pixels, and variable computer controlled shear optics. Illumination is provided by an integrated diode laser array. It can typically inspect an area $300 \times 300 \mathrm{~mm}^{2}$ under 20 seconds with a sensitivity of $0.03 \mu \mathrm{m}$ in ratio to the shear distance. Dantec Dynamics also sells a portable vacuum hood shearography system, Q-810. The company Steinbichler has industrialized a composites inspection system called Isismobile. Their products target essentially composite components inspection, such as sandwich structures with honeycomb or foam core, GlareTM, aluminium composite, and other compound materials.

\subsubsection{Challenges of Shearography}

The challenges of a technique are where the current limitations of this technique lie. Shearography is very popular as qualitative tool for nondestructive testing among industrials. One of the main challenges of shearography is to bring this optical method to the level of quantitative tool for the industry.

Usually, each measurement taken with a shearographic interferometer contains both the in-plane strain and the out-of-plane component. It is now possible to separate and extract them simultaneously or sequentially accordingly to the configuration. The first derivate of out-of-plane displacement can be easily separated by manipulating the illumination while the in-plane component need a multiple-source configuration to be extracted. Simultaneous measurements have already been achieved with success but in a relatively complex manner. Multiple-axis measurements are easily performed sequentially but any simple and efficient solution has been presented yet for doing it simultaneously. 
Large rigid body motions of the test specimen during measurement produce a decorrelation of speckle patterns which reduces the fringe visibility and in the worst case it leads to a complete loss of fringes. The range of measurement in ESPI techniques is limited by speckle pattern decorrelation and the camera

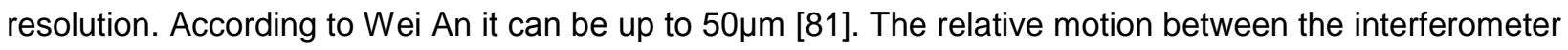
and test specimen could also lead to fringe degradation. Shearography depends on test specimen deformation to reveal subsurface defects. For that, it is necessary to impose stresses, or additional stresses on the part. For measurements performed outside laboratory conditions, the background lighting could add unwanted light onto the sensor. Shearography depends on the surface condition of the test object, which needs to be ideally of good reflectance and diffusely scatter the incident laser light. Certain materials, like composites, parts having black surfaces, metallic surfaces, or polished surfaces make the optical power delivered to the sensor too low to perform any numerical manipulation.

Shearography is a method for direct measurement of derivatives of surface displacement. It is a relative measurement in which two sets of images are recorded for different loading conditions of the test specimen. Dynamic phase shifting yields the absolute phase change and makes phase unwrapping obsolete but the method requires the recording of many intensity images during the deformation of the object [82]. Any discontinuity appearing during the recording would corrupt the measurement. The absolute phase determination still remains a challenge.

Another challenge of shearography is its high sensitivity. A too high sensitivity can be reduced by decreasing the shear distance and opposingly a low sensitivity can be enlarged by increasing the shear distance. Even if the measuring sensitivity of shearography is adjustable by changing the shearing distance, potential conditions could result in too many fringes thus making defect detection difficult. This limitation is one more negative aspect of speckle interferometry techniques including shearography which makes difficult to apply them for in situ measurements.

\subsection{Research Objectives and Proposed Method}

This thesis illustrates the use of multiple separate-color measurements using shearography as an example. This technique measures out-of-plane rotations within the measured area. It is a popular technique because its parallel path optical configuration and its low sensitivity to rigid-body motions makes it relatively stable compared with other ESPI measurement types $[9,10]$ and thus more practical for field use. A multi-color shearography setup for full-field quantitative measurements of slope in two orthogonal directions is presented here. The setup uses two of the three color measurements available within a Bayer type camera. The third available color measurement is used to determine absolute phase. The construction of the proposed shearography system is described and its capabilities are demonstrated through some example measurements. 
The main objective consists to prove the concept of simultaneous ESPI measurements using multiple wavelengths and a color camera. Shearography has the same advantages as ESPI but unlike ESPI, it is less sensitive to inspection environments and does not require particular vibration isolation. Overall, shearography is more practical than electronic speckle pattern interferometry for in situ inspection and nondestructive testing in an industrial environment. This strong asset explains the choice made to use this technique for demonstrating the possibility of performing multiple-wavelength measurements using a color camera. The phase map reconstruction remains similar between shearography and ESPI and the colored signals can be processed the same way for both technique. However, the phase interpretation depends essentially from the interferometer configuration.

A shearography setup involving simultaneous illumination by three independent light sources of different wavelengths has been developed. A RGB color camera allows to capture simultaneously the colored signals originating from the three laser sources. By individually reading the RGB signals it is possible to separate the three intensity distribution patterns, measured by the CCD image sensor, and thus achieve three simultaneous and independent measurements. Shearography is normally performed using a setup based on a Michelson interferometer, which is a very compact and simple solution that allows an easy alignment of the optical components. The new concept includes one dichroic filter, one beamsplitter cube, and two tilt mirrors with one that is mounted on piezo elements. This concept coupled with the colored signals and a color camera creates an extremely compact dual-axis shearographic interferometer. Two colors measures two mutually orthogonal first derivatives of out-of-plane displacement at every pixel within the optical field.

ESPI is a relative measuring technique and provides only relative phase values. The absolute phase can be extracted via the two-wavelength method, where the third available color is used redundantly with one of the two first colors. The two wavelengths used for determination of the absolute phase measure the exact same quantity. This absolute phase is determined according to only one of the two available axes.

The sensitivity for shearography can be adjusted by varying either the wavelengths or the shear distance. The wavelengths are well known and usually fixed, without taking into account drifts arising from environmental disturbances such as temperature changes. The shear distance is the most important parameter to control and must be accurately measured. Digital image correlation is chosen to measure it. The shear distance is supposedly uniform across the entire image but in reality it is not the case. An algorithm is developed to correct for non-uniform shear using measurements of two mutually orthogonal signals.

The new dual-axis interferometer gives the possibility of reconstructing the object's surface after deformation. Numerical summation of both orthogonal phase maps yields to the deformed shape of the test specimen. The dual-axis shearographic interferometer records two pair of shifted images or four images encompassed in a single frame. If an image of a piece with a hole on its surface was captured, 
the hole would appear three times and not four because the two interferometers have a common reference. The summation of two phase maps obtained in two orthogonal directions gives the displacement field and at the same time it removes the image doubling caused by the two pair of shifted images.

Finally, the effective operation of this new dual-axis shearographic interferometer is demonstrated by comparing measurements using known deformations, thus validating the use of a color camera for doing simultaneous ESPI measurements. This capability is possible by using the three separate color measurements available with a Bayer style camera. The technique is valuable because it allows the use of much simpler and more compact optical equipment and because it provides multiple independent measurements that are automatically coordinated in both time and space.

\subsection{Overview}

The present work is organized as follows: Chapter 2 offers a brief explanation on how color cameras work. The color filtering, separation, and calibration are presented.

Chapter 3 provides an overall explanation of ESPI technique. The principle and procedure of ESPI technique, phase stepping algorithm, and phase map unwrapping are explained. Basically, the principal techniques used in electronic speckle pattern interferometry and shearography to determine the phase from the recorded intensity are presented in this chapter.

Chapter 4 delivers a general explanation of shearography technique. It is shown how the out-of-plane component is calculated and also how the in-plane component is isolated. The dual-axis shearographic interferometer is described in details. Then the correction for non-uniform shear is explained followed by the absolute phase determination. Finally the deformed shape measurement is introduced.

Chapter 5 reports the experimental result from dual-axis shearographic measurements. These results are then validated by comparison with known deformations.

Chapter 6 contains the conclusions, which summarize the main achievements of this work. Then the contributions and limitations are presented. Finally, recommendations are given focusing on how the method could be further improved. 


\section{Chapter 2. Color Measurements}

\subsection{Color Cameras}

Current digital cameras are implemented with two main sensor types: Complementary Metal-OxideSemiconductor (CMOS) sensors and Charge-Coupled Device (CCD) sensors. CCD sensors are predominant in the field of speckle interferometry.

CCD sensors were introduced in 1969 by researchers at Bell Laboratories. They were specifically designed to be used in cameras for replacing photographic film. A CCD is made of a thin silicon wafer divided into thousands or millions pixels that capture light. When photons of light hit the pixels, photoelectric effect happens and converts incident photons into charges. The charges are then converted to voltage levels. The output voltage from each pixel is dependent on the number of photons that penetrated the silicon surface and it can be finally converted to a digital signal.

The CMOS technology was patented by Frank Wanlass in 1963. Both CMOS sensors and CCD have the same function, they convert light into electrons. A CMOS sensor has a different architecture and it reads information directly from each pixel instead of row by row because each pixel has its own charge-tovoltage conversion and amplifier. Both sensors carry out same steps. The collected light is converted into charges, which are then accumulated, transferred, and finally converted into a voltage. This voltage is then amplified before it is sent to the camera circuitry.

CMOS sensors are cheaper than CCDs, mainly because they are easier to manufacture even if each pixel has its own amplifier. Microelectronics is directly incorporated on the sensor as shown in Figure 3, while for CCD a separate amplifier and other electronic components are needed and this adds to the cost of the already expensive sensor. CMOS sensors have a lower light sensitivity, they are physically larger, and are more prone to noise. The low light sensitivity required greater amplification of the signal thus leading to more noise and since there are millions of individual amplifiers, the problem is intensified.

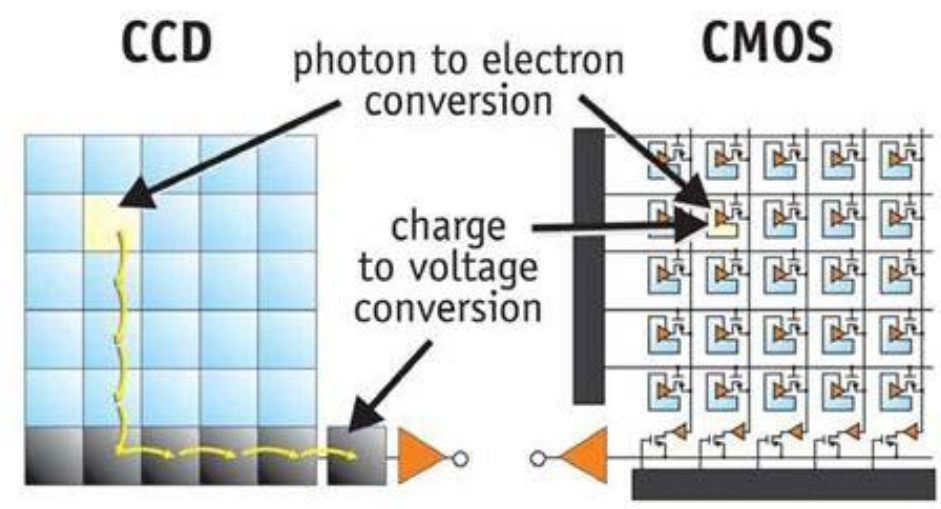

Figure 3 - CCDs move photogenerated charge from pixel to pixel and convert it to voltage at an output node. CMOS imagers convert charge to voltage inside each pixel (adapted from Stefano Meroli). 
Helmers and Schellenberg compared the use of CMOS and CCD sensors in speckle interferometry [83]. According to their study, spatial resolution, temporal noise, and sensitivity are comparable for both types of cameras. Differences between the cameras occur in the pattern noise and the characteristic curve. The pattern noise of the CMOS cameras is considerably higher than that of the CCD cameras. The characteristic curve of the CMOS camera can be changed to a more logarithmic course by adjusting electronic parameters. This allows the recording of light fields with large brightness differences without pixel saturation and blooming.

For this research, a Prosilica GE680C color camera manufactured by Allied Vision Technologies has been used. The GE680C is a fast CCD camera with Gigabit Ethernet interface with more than $200 \mathrm{fps}$. Its CCD sensor captures images with $7.4 \mu \mathrm{m}$ square pixels and a resolution of $640 \times 480$ pixels. A Bayer Filter enables colored recordings.

\subsubsection{Bayer Filter}

An image recorded with a CCD sensor is black and white, but by placing a colored filter in front of each pixel, color information is collected at every pixel. Red, green, and blue filters are essentially used to correspond to the human perception of colors. The most common color filter array is the Bayer Filter [45]. This color filter array, shown in Figure 4, has a pattern that alternates rows of red and green filters with rows of blue and green filters. There are twice as many green filters as red or blue filters.

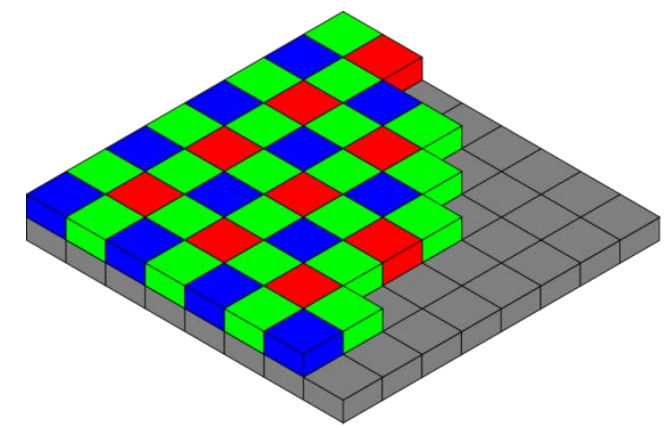

Figure 4 - The Bayer arrangement of color filters on the pixel array of an image sensor (Wikipedia).

Since each pixel measures only one single color, the other colors must be estimated using information from the entire color array to reconstruct a full color image. The process that estimates the missing two color values at each pixel is known as demosaicing. The simplest method available is a linear interpolation of the known color values from the nearest neighboring pixel as illustrated in Figure 5. A large variety of methods exists such as: pixel doubling interpolation, bilinear interpolation, constant huebased interpolation, median-based interpolation, gradient based interpolation, or adaptive color plane interpolation. 

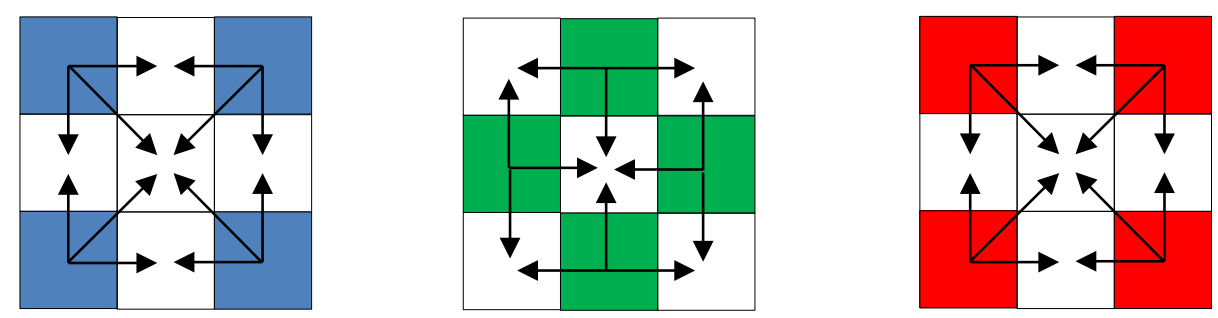

Figure 5 - Demosaicing estimates the missing color pixel value by interpolating the neighboring values.

The Bayer mosaic pattern opens the possibility to record three independent signals. Even if each color does not reach the full sensor resolution, demosaicing performed with a linear interpolation allows acquisition of a full single color frame for each color. Interpolation is only an approximation; therefore an image will always lose some quality each time interpolation is applied. One direct solution to overcome this problem is to simply use CCD sensors with higher resolution, which moves the problem to a more financial aspect.

Another alternative to collect three independent signals exists with the advantage for doing it at each pixel. Knipp, Stiebig, and Wagner from the Institute of Photovoltaics in Jülich, Germany, developed color sensors based on vertical integrated thin film structures where color separation is realized by the wavelength dependent absorption in the depth of the material [84]. Their system is based on amorphous silicon layers and optical filters are not required anymore. In 2002, the company Foveon, Inc. announced the Foveon X3 three-layer silicon sensor. The Sigma SD9 is the first digital camera to use this full-color multi-layer sensor technology.

\subsection{Color Calibration}

The sensor of a typical color camera consists of a grid of pixels on which color filters have been fixed in a mosaic pattern. The Bayer pattern is the most common design, with a sequence of $50 \%$ green pixels and $25 \%$ each of red and blue pixels. Figure 6 shows the transmission curves for the pixel color filters for a typical color camera. 


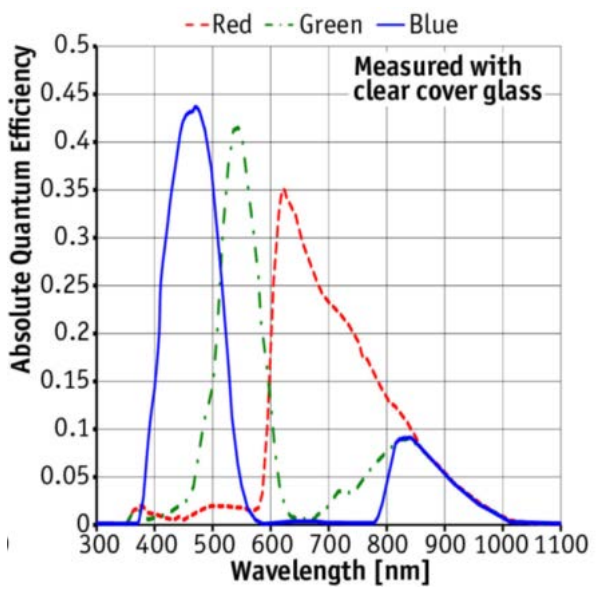

Figure 6 - Absolute quantum efficiency graph of the Prosilica GE680C color camera - Allied Vision Technologies.

These filters are designed to be wide and have significant overlaps so that the camera is able to represent realistically the full range of colors present in a general image. However, for the present purpose a much tighter color response is needed. For good results, each color measurement should be entirely independent; overlap ("cross-talk") between color responses is to be avoided. This behavior can be achieved by simultaneous considerations of the colored signals. For example, consider illumination by a beam of color 1 (red) only, with average intensity $I_{1}$. The measured responses of the Red, Green and Blue pixels are $R_{1}, G_{1}, B_{1}$ where:

$$
R_{1}=A_{11} I_{1} \quad G_{1}=A_{21} I_{1} \quad B_{1}=A_{31} I_{1}
$$

and $A_{i j}$ is the response of pixel color $i$ to illumination color $j$. In general, the numerical value of $I_{1}$ is not known. However, it is sufficient for the present purpose to work in terms of scaled quantities and to normalize the calibration coefficients $A_{i j}$ :

$$
a_{11}=\frac{A_{11}}{\max \left(A_{11}, A_{21}, A_{31}\right)} \quad a_{21}=\frac{A_{21}}{\max \left(A_{11}, A_{21}, A_{31}\right)} \quad a_{31}=\frac{A_{31}}{\max \left(A_{11}, A_{21}, A_{31}\right)}
$$

Thus, the largest of $a_{11}, a_{21}, a_{31}$ equals 1 . Similarly, with beams of colors 2 or 3 only, subscript "2" or "3" replaces subscript " 1 " above. When all three beams are present simultaneously, the measured responses are:

$$
\left[\begin{array}{l}
m_{1} \\
m_{2} \\
m_{3}
\end{array}\right]=\left[\begin{array}{l}
R_{1}+G_{1}+B_{1} \\
R_{2}+G_{2}+B_{2} \\
R_{3}+G_{3}+B_{3}
\end{array}\right]=\left[\begin{array}{lll}
a_{11} & a_{12} & a_{13} \\
a_{21} & a_{22} & a_{23} \\
a_{31} & a_{32} & a_{33}
\end{array}\right]\left[\begin{array}{l}
\mathbb{I}_{1} \\
\mathbb{I}_{2} \\
\mathbb{I}_{3}
\end{array}\right]
$$

which becomes in matrix form:

$$
a \mathbb{I}=m
$$


The individual beam intensities II can be recovered from the measured responses $m$ by matrix solution. The scaling in Equation (2) causes the computed II values to be in the same range as the original pixel readings.

\subsection{Discussion}

In this chapter, the operation of CCD cameras and a comparison with CMOS cameras have been briefly introduced. CCD sensors have less noise than CMOSs sensors. The concept of recording different colors with a Bayer type camera has been presented. That type of filter enables to record simultaneously three independent colored signals, which could be used in interferometry for measuring simultaneously deformation in two or potentially three different directions. Since each colored pattern has missing values, a demosaicing procedure has been illustrated. Cross-talks between color responses exist and need to be corrected using a calibration method. 


\section{Chapter 3. Electronic Speckle Pattern Interferometry}

\subsection{The Nature of Light and the Laser Speckle Phenomenon}

The speckle effect occurs when a beam of coherent light, originating from a source such as a laser, illuminates an optically rough surface. The roughness of the surface needs to be at least similar in scale to the wavelength of the illuminating light [85]. Surfaces with roughness smaller than the wavelength being used lead to specular reflection, where the angle of incidence is equal to the angle of reflection. Rough surfaces lead to diffuse reflection, where the light is scattered in every direction. Steinchen mentioned in his book [6] that operation of superfinishing, lapping, polishing, honing, and grinding create an average roughness that is too fine, between 0.05 and $0.4 \mu \mathrm{m}$, for interferometric measurements. Surfaces with bright color reflect more light and often dark surfaces need to be painted with a thin layer of white paint to improve surface reflectivity. A granular structure composed of dark and bright spots of random shape and size may be observed from anywhere nearby once the light is reflected from the rough surface. The incoming light hits the surface to be then scattered in every direction. When two rays of light of same wavelength intersect each other, interference occurs and when they interfere on a plane such as a CCD sensor, a speckle pattern made of dark and bright spots is formed. In a statistical point of view, the speckle pattern is a three-dimensional interference pattern. Figure 7 shows a two-dimensional representation of the speckle statistics.

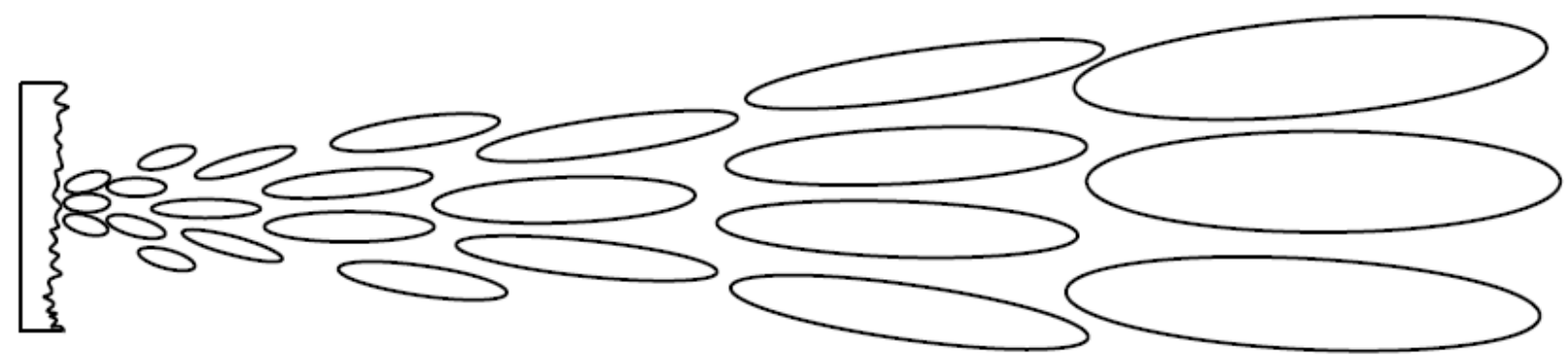

Figure 7 - Geometric representation of the speckle statistics (adapted from [86]).

The speckle phenomenon can be observed in two different ways as presented in Figure 8. As long that there is no imaging lens in the system, a speckle pattern in free space is called objective speckle pattern and cannot be observed directly. The only way to observe objective speckle patterns is to use a photographic film, a scanning picture-sensing detector, or a detector array without any lens. Whenever a lens is used, the observed speckle pattern is called subjective speckle pattern. 

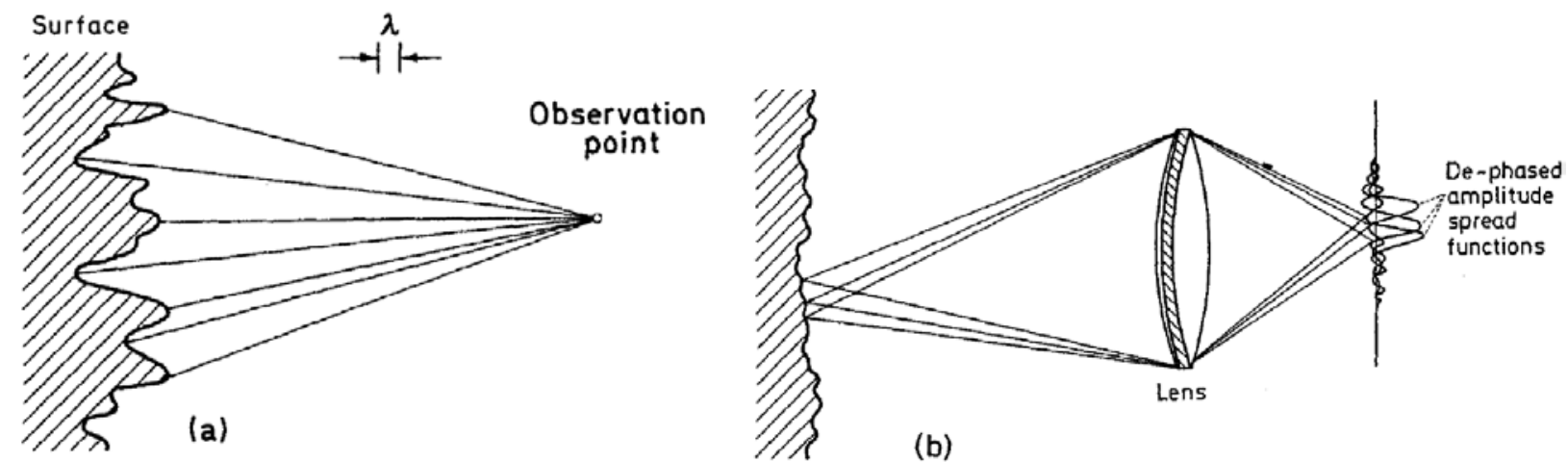

(b)

Figure 8 - Physical origin of speckle for (a) free-space propagation, (b) an imaging system (adapted from [87])

Light can be thought of as a wave propagating through space. When at least two light waves of same frequency are superposed, interference occurs. The bright spots observed in a speckle pattern are formed when several interfering light waves are in-phase and the dark spots are the result of interferences of out-of-phase waves. A speckle pattern is not composed of only pure black spots, destructive interference, or pure white spots, constructive interference, but it is composed of shades of grey because any case in-between is plausible. The measured intensity depends on whether the two waves reinforce or cancel each other. The resultant complex amplitude at any point in the interference pattern is the sum of the complex amplitudes of these two waves or beam of light, which can be written as [88]:

$$
A=A_{1}+A_{2}
$$

where $A_{1}=a_{1} \exp \left(-i \phi_{1}\right)$ and $A_{2}=a_{2} \exp \left(-i \phi_{2}\right)$ are the complex amplitudes of the two waves. The resultant intensity is, therefore,

$$
\begin{aligned}
I & =|A|^{2} \\
& =\left(A_{1}+A_{2}\right)\left(A_{1}^{*}+A_{2}^{*}\right) \\
& =\left|A_{1}\right|^{2}+\left|A_{2}\right|^{2}+A_{1} A_{2}^{*}+A_{1}^{*} A_{2} \\
& =I_{1}+I_{2}+2\left(I_{1} I_{2}\right)^{1 / 2} \cos \Delta \phi
\end{aligned}
$$

where $I_{1}$ and $I_{2}$ are the intensities due to the two waves acting separately, and $\Delta \phi=\phi_{1}-\phi_{2}$ is the phase difference between them.

If the two waves are derived from the same source, so that they have the same phase at the origin, the phase difference $\Delta \phi$ corresponds to an optical path difference:

$$
\Delta L=(\lambda / 2 \pi) \Delta \phi
$$


The ESPI technique consists in measuring surface displacements. It can be achieved qualitatively by subtracting speckle pattern recorded, through a CCD camera, before and after deformation of the test specimen. Quantitative measurements are completed by using a multiple-step stepping technique, where several images are recorded before deformation to determine phase difference $\Delta \phi_{1}$ and after deformation to recover phase difference $\Delta \phi_{2}$. The change of relative phase difference $\Delta \varphi$ between these two angles is:

$$
\Delta \varphi=\Delta \phi_{2}-\Delta \phi_{1}
$$

If $\Delta \varphi$, the relative phase difference between the two different states, varies linearly across the image, the intensity varies cosinusoidally, generating alternating light and dark interference fringes. These fringes are loci of constant phase difference.

An ESPI arrangement is characterized by its measurement sensitivity direction. The sensitivity vector $\overrightarrow{k_{s}}$, which depends on the direction of illumination and observation, is determined by the geometry of the setup. The relative phase change $\Delta \varphi$ can be related to the surface deformation $\vec{d}$, illustrated in Figure 9 , and the sensitivity vector $\overrightarrow{k_{s}}$ :

$$
\Delta \varphi=(2 \pi / \lambda) \cdot \overrightarrow{k_{s}} \cdot \vec{d}
$$

Equation (9) illustrates how the deformations $\vec{d}$ are obtained by measuring the relative phase change $\Delta \varphi$ between tow given states.

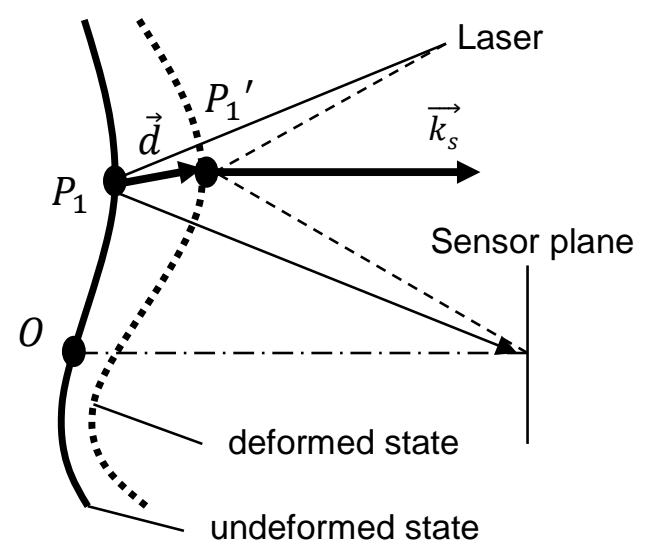

Figure 9 - Change in the optical path length due to surface deformation.

\subsection{A Typical ESPI Optical Setup}

An electronic speckle pattern interferometer aims to measure surface displacements. These 
measurements depend on the induced phase difference and on the interferometer's sensitivity vector, which is governed by the geometry of the optical arrangement and the illuminating sources. The interferometer can be arranged to be sensitive to out-of-plane displacements, or in-plane displacements.

\subsubsection{In-Plane Sensitive ESPI System}

Generally, deformation on a specimen occurs in three dimensions. Three dimensional measurements are divided into in-plane and out-of-plane measurements. When working with optics, the $Z$ axis is determined by the direction the light travels. In Figure 10, the light travels from the object surface to the camera. In that case, the optical arrangement is seen from top, the $\mathrm{Y}$-axis corresponds to the vertical direction and the $\mathrm{X}$-axis is along the horizontal direction. The surface displacement components along the Z-axis are out-of-plane components, and those along $\mathrm{X}$-axis and $\mathrm{Y}$-axis are in-plane components.

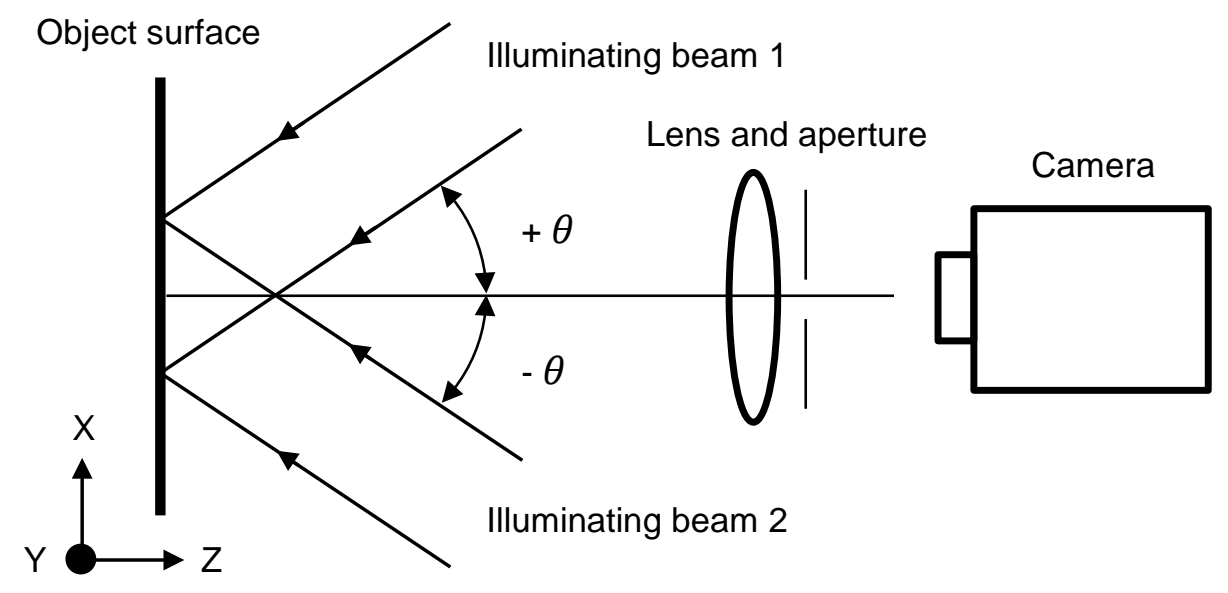

Figure 10 - Optical configuration of an in-plane ESPI system.

Figure 10 shows an in-plane sensitive ESPI arrangement, which measures in-plane surface displacements along the X-axis. Usually, the two illuminating beams originate from the same coherent source to be then divided into two parts using a beamsplitter. The laser and some other optical elements have been omitted for simplicity. One of the beams might be deliberately altered by placing in its path a mirror attached to a piezo element to perform quantitative measurement with a phase-stepped method. This modified beam is called a reference beam. The second beam, or the illumination beam shine the object surface at a negative angle $\theta$ with respect to the viewing direction and lying in the X,Z-plane. The reference beam illuminates the object at the same angle but in opposite directions. Light from both beams is scattered in every directions. A portion of the light is reflected towards the CCD camera and interferes onto the camera sensor thus generating a speckle pattern. The intensity of several speckle patterns is recorded before and after deformation of the test specimen. Using phase-stepping allows to retrieve the 
relative phase change induced by the imposed deformation and displacements are then calculated according the measurement sensitivity of the system.

A point on the object surface under investigation moves by $\vec{d}=d_{x} \vec{\imath}+d_{y} \vec{\jmath}+d_{z} \vec{k}$ from its original position upon deformation.

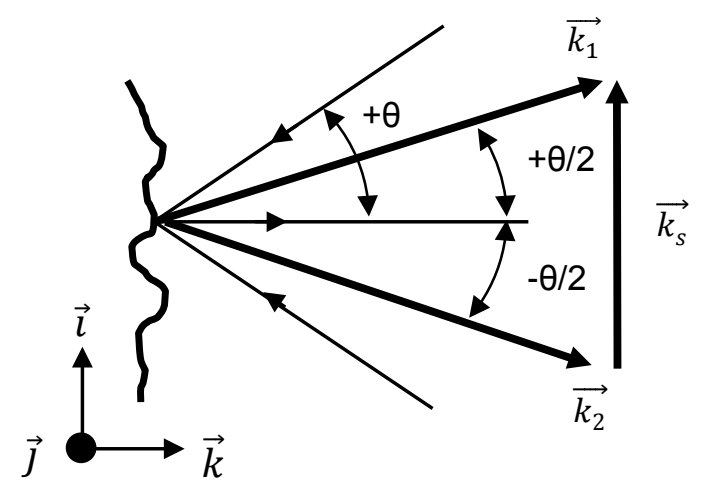

Figure 11 - In-plane sensitivity vector.

The sensitivity vector is the bisector of the angle between the illumination and viewing direction and the resultant sensitivity vector, shown in Figure 11, can be expressed as:

$$
\begin{aligned}
\overrightarrow{k_{1}} & =\sin \frac{\theta}{2} \vec{\imath}+\cos \frac{\theta}{2} \vec{k} \\
\overrightarrow{k_{2}} & =-\sin \frac{\theta}{2} \vec{\imath}+\cos \frac{\theta}{2} \vec{k} \\
\overrightarrow{k_{s}} & =\overrightarrow{k_{1}}-\overrightarrow{k_{2}}=2 \sin \frac{\theta}{2} \vec{\imath}
\end{aligned}
$$

where $\overrightarrow{k_{1}}$ is the sensitivity vector for the first source of illumination and $\overrightarrow{k_{2}}$ is the sensitivity vector for the second beam of light. Upon deformation, the change in optical path length has two components, one before the object surface and one after the surface. The relative phase change is:

$$
\begin{aligned}
& \Delta \varphi=2 \cdot\left(\frac{2 \pi}{\lambda}\right) \cdot \overrightarrow{k_{s}} \cdot \vec{d} \\
& =\left(\frac{4 \pi}{\lambda}\right) \cdot 2 \sin \frac{\theta}{2} \vec{\imath} \cdot\left(d_{x} \vec{\imath}+d_{y} \vec{\jmath}+d_{z} \vec{k}\right) \\
& =\left(\frac{8 \pi}{\lambda}\right) \cdot \sin \frac{\theta}{2} \cdot \cos \frac{\theta}{2} \cdot d_{x} \\
& =\left(\frac{4 \pi}{\lambda}\right) \cdot \sin \theta \cdot d_{x}
\end{aligned}
$$

Equation (13) shows that only the displacement component, which depends on the resultant sensitivity vector, contributes to the relative phase change. 


\subsubsection{Out-of-Plane Sensitive ESPI System}

Figure 12 shows a mainly out-of-plane sensitive ESPI system. As for the in-plane ESPI system, the laser and some other optical elements are missing in the graph.

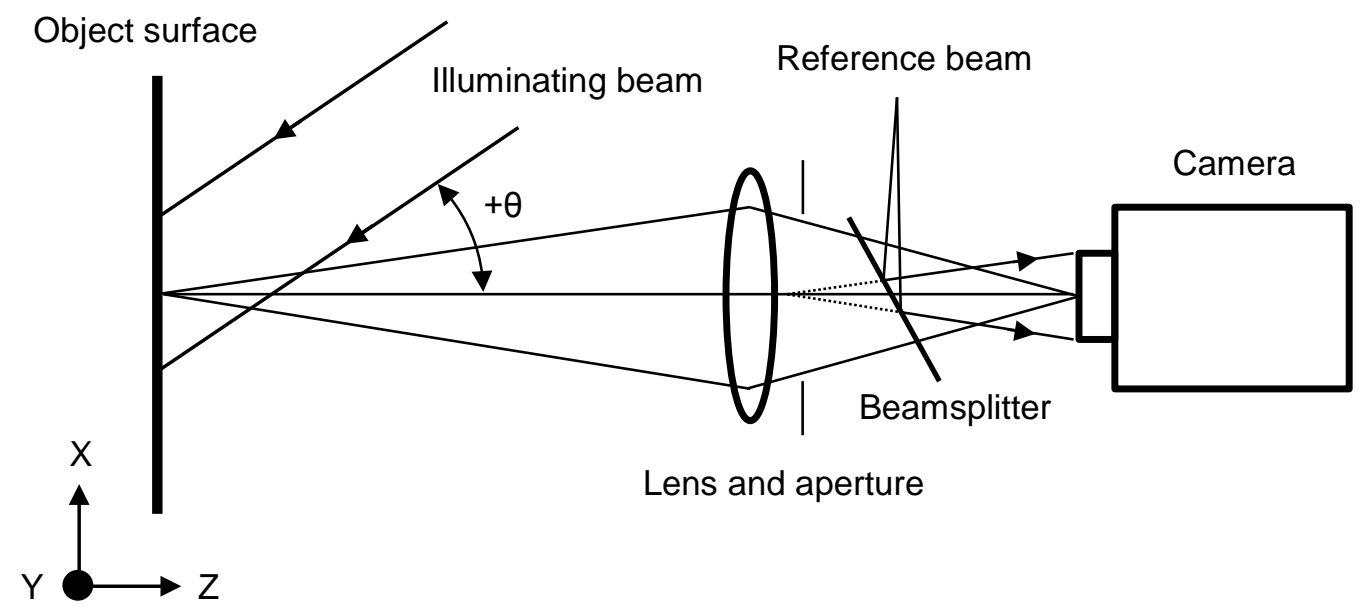

Figure 12 - Optical configuration of an out-of-plane ESPI system.

The out-of-plane arrangement looks similar to the in-plane interferometer configuration. The main difference is the illumination geometry, where in the out-of-plane case only the illumination beam shines the object surface while the reference beam is directly brought onto the camera sensor. The illumination angle $\theta$ must be small to decrease the in-plane sensitivity and to maximize the out-of-plane sensitivity. The reference beam is introduced by means of a beamsplitter and is aligned with the object beam. The image recording process and the phase calculation are exactly the same as described for the in-plane system.

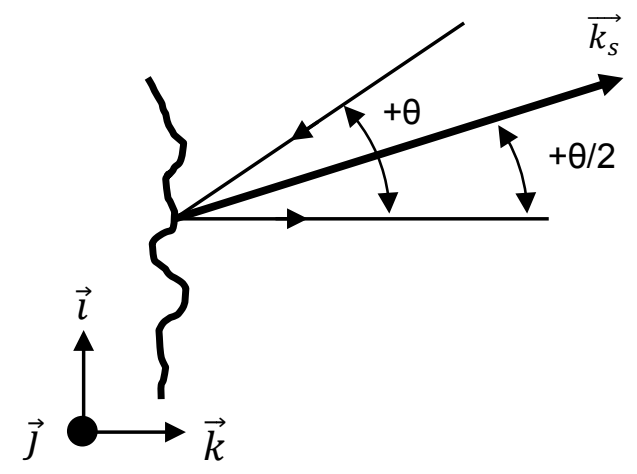

Figure 13 - Out-of-plane sensitivity vector. 
The sensitivity vector is defined as the bisector of the angle between the illumination and viewing direction and the resultant sensitivity vector, shown in Figure 13, can be expressed as:

$$
\overrightarrow{k_{s}}=\sin \frac{\theta}{2} \vec{\imath}+\cos \frac{\theta}{2} \vec{k}
$$

Upon deformation, the optical path length is affected two times, before the object surface and also after the surface. The relative phase change is:

$$
\begin{aligned}
& \Delta \varphi=2 \cdot\left(\frac{2 \pi}{\lambda}\right) \cdot \overrightarrow{k_{s}} \cdot \vec{d} \\
& =\left(\frac{4 \pi}{\lambda}\right) \cdot\left(\sin \frac{\theta}{2} \vec{\imath}+\cos \frac{\theta}{2} \vec{k}\right) \cdot\left(d_{x} \vec{\imath}+d_{y} \vec{\jmath}+d_{z} \vec{k}\right)
\end{aligned}
$$

Because $\theta$ is small the in-plane components are neglected, thus the relative phase change becomes:

$$
\begin{aligned}
& \Delta \varphi=\left(\frac{4 \pi}{\lambda}\right) \cdot \cos \frac{\theta}{2} \cdot \cos \frac{\theta}{2} \cdot d_{x} \\
& =\left(\frac{2 \pi}{\lambda}\right) \cdot(1+\cos \theta) \cdot d_{z}
\end{aligned}
$$

If the illumination is normal to the object surface and lies along the viewing direction then the phase change becomes:

$$
\Delta \varphi=\left(\frac{4 \pi}{\lambda}\right) \cdot d_{z}
$$

Equation (17) shows that only the displacement component, which depends on the sensitivity vector, contributes to the relative phase change.

\subsection{Phase Stepping}

The ESPI technique is or was mainly used for qualitative measurements, where the recorded intensities are subtracted from each other to yield a fringe pattern. For quantitative analysis, phase stepping is required. The intensity resulting from interference of two beams of light captured after surface deformation can be expressed as:

$$
J=J_{1}+J_{2}+2\left(J_{1} J_{2}\right)^{1 / 2} \cos \Delta \phi_{2}
$$

Equation (18) can be simplified and rewritten as:

$$
J=A+B \cos \Delta \phi_{2}
$$


where $A=J_{1}+J_{2}$ is the mean intensity, $B=2\left(J_{1} J_{2}\right)^{1 / 2}$ is the amplitude, and $\Delta \phi_{2}$ is the phase difference after surface deformation.

There are three unknown and at least three equations are needed to retrieve the phase difference. A fourstep method requires to record four speckle pattern to obtain four intensities $I_{1}, I_{2}, I_{3}, I_{4}$ before deformation and $J_{1}, J_{2}, J_{3}, J_{4}$ after deformation and the relative phases angles $\Delta \phi_{1}, \Delta \phi_{2}$. A four-step algorithm has the advantages of decreasing the sensitivity to the phase step errors and also of allowing data averaging.

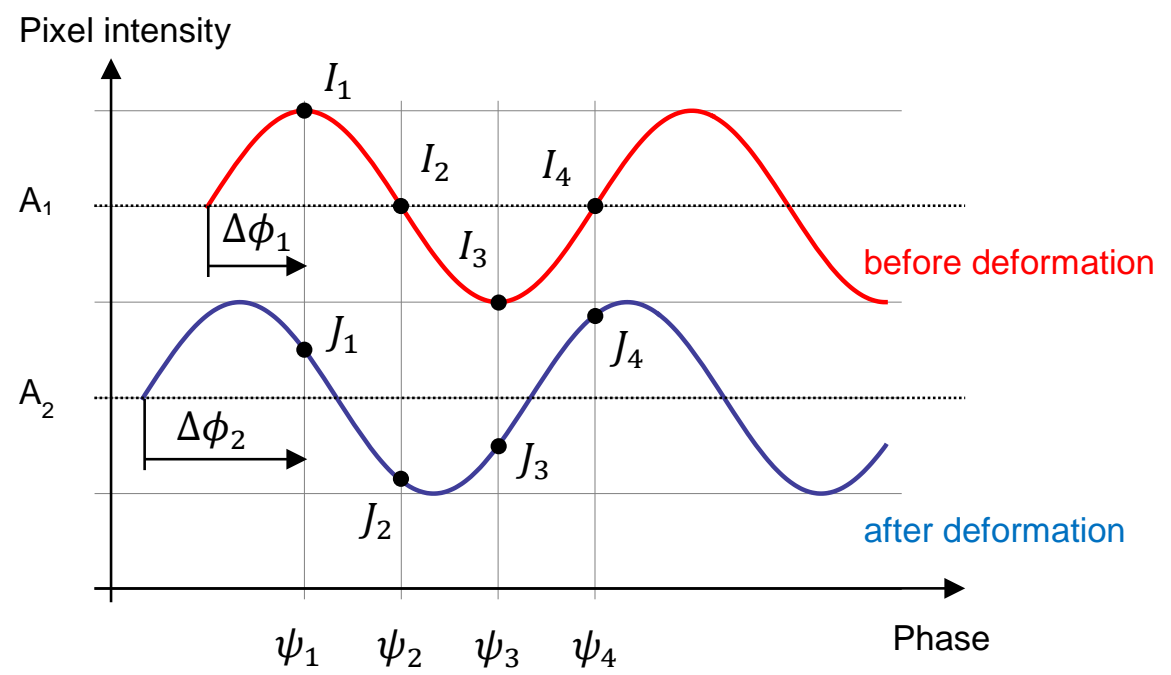

Figure 14 - Sinusoidal intensity profile of a single pixel before and after deformation.

Figure 14 shows how the recorded intensity for every single pixel makes possible to retrieve the sinusoidal intensity profile and simultaneously to extract the phase difference. The process consists in stepping the phase angle by altering the reference beam with a mirror mounted on a piezo element. The piezo is moved between each image recording such that the phase angle changes by $90^{\circ}$.

Considering the images before deformation, the intensity distribution can be written as:

$$
I_{n}=A+B \cos \left(\Delta \phi+\psi_{n}\right)
$$

with $\psi_{n}$ being a known set of phase steps, usually in $90^{\circ}$ increments. Equation (20) can also be expressed using the additive cosine identity as:

$$
I_{n}=C_{1}+C_{2} \sin \psi_{n}+C_{3} \cos \psi_{n}
$$

where $C_{1}=A, C_{2}=-B \sin \Delta \phi$, and $C_{3}=B \cos \Delta \phi$. The system of equations can be rewritten as: 


$$
\begin{gathered}
{\left[\begin{array}{c}
I_{1} \\
\vdots \\
I_{n}
\end{array}\right]=\left[\begin{array}{ccc}
1 & \sin \psi_{1} & \cos \psi_{1} \\
\vdots & \vdots & \vdots \\
1 & \sin \psi_{n} & \cos \psi_{n}
\end{array}\right]\left[\begin{array}{l}
C_{1} \\
C_{2} \\
C_{3}
\end{array}\right]} \\
d=G m
\end{gathered}
$$

where: $d=\left[\begin{array}{c}I_{1} \\ \vdots \\ I_{n}\end{array}\right], G=\left[\begin{array}{ccc}1 & \sin \psi_{1} & \cos \psi_{1} \\ \vdots & \vdots & \vdots \\ 1 & \sin \psi_{n} & \cos \psi_{n}\end{array}\right]$, and $m=\left[\begin{array}{c}C_{1} \\ C_{2} \\ C_{3}\end{array}\right]$.

Forming a least squares solution by minimizing the error yields:

$$
G^{T} G m=G^{T} d
$$

Simplifying Equation (24) yields:

$$
\left[\begin{array}{ccc}
n & \sum \sin \psi_{n} & \sum \cos \psi_{n} \\
\sum \sin \psi_{n} & \sum \sin ^{2} \psi_{n} & \sum \sin \psi_{n} \cos \psi_{n} \\
\sum \cos \psi_{n} & \sum \sin \psi_{n} \cos \psi_{n} & \sum \cos ^{2} \psi_{n}
\end{array}\right]\left[\begin{array}{l}
C_{1} \\
C_{2} \\
C_{3}
\end{array}\right]=\left[\begin{array}{c}
\sum I_{n} \\
\sum \sin \psi_{n} I_{n} \\
\sum \cos \psi_{n} I_{n}
\end{array}\right]
$$

Solving Equation (25) using a four-step method with $\psi_{n}=\left[0^{\circ}, 90^{\circ}, 180^{\circ}, 270^{\circ}\right]$ leads to:

$$
\tan \Delta \phi_{1}=\frac{I_{2}-I_{4}}{I_{1}-I_{3}} \text { and } \tan \Delta \phi_{2}=\frac{J_{2}-J_{4}}{J_{1}-J_{3}}
$$

To recap, the relative phase change $\Delta \varphi$ between these two angles is:

$$
\Delta \varphi=\Delta \phi_{2}-\Delta \phi_{1}
$$

\subsubsection{Multiple-Wavelength Phase Stepping}

A common five-step scheme [89] is used here with $\psi_{n}=\left[0^{\circ}, 90^{\circ}, 180^{\circ}, 270^{\circ}, 360^{\circ}\right]$ as step angles. Using these values, the phase difference between the illumination and reference beams is:

$$
\tan \Delta \phi_{1}=\frac{7\left(I_{2}-I_{4}\right)}{4 I_{1}-I_{2}-6 I_{3}-I_{4}+4 I_{5}}
$$

The phase difference is extracted according to Equation (28) while the object under analysis is in its reference state. Then, the same sequence of stepped measurements is made while the object is in the deformed state. The phase change between the initial and deformed states can be determined by subtraction of the phase values at each pixel and a phase change map can be formed. For practical use, the phase map can be further processed by filtering [90] and unwrapping [91]. 
ESPI measurements implemented with phase-stepping for phase determination traditionally need only one coherent light source, an interferometer, and one monochrome camera. The use of multiple colors in the ESPI technique requires simultaneous determination of the phases of the corresponding colors. The red, green, and blue colors used have greatly different frequencies and so do not interfere with each other. In other words, working these colors allows simultaneous measurement of three independent phase differences. The ESPI setup used in this research uses a red laser of 660nm, a green laser of $532 \mathrm{~nm}$, and blue laser of $473 \mathrm{~nm}$. A $90^{\circ}$ phase step on the green color will not be equivalent of a $90^{\circ}$ phase step on a red or blue color. Here, the green signal is taken as a reference and is stepped at a phase of $90^{\circ}$, thus stepping the red signal at a lower angle reduced by the ratio of the green and red wavelengths, $\psi_{n}=\left[0^{\circ}, 90^{\circ}, 180^{\circ}, 270^{\circ}, 360^{\circ}\right] \times 532 / 660$. Similarly, the stepping of the blue signal is increased by the ratio of the green and blue wavelengths, $\psi_{n}=\left[0^{\circ}, 90^{\circ}, 180^{\circ}, 270^{\circ}, 360^{\circ}\right] \times 532 / 473$. For these non-orthogonal steps, the phase can be determined by solving Equation (25) where,

$$
\Delta \phi=\arctan \left(\frac{C_{2}}{C_{3}}\right)
$$

Equations (25) and (29) reduce to Eq.(28) for the case where $\psi_{n}=\left[0^{\circ}, 90^{\circ}, 180^{\circ}, 270^{\circ}, 360^{\circ}\right]$. For the general case of a non-orthogonal angle $\psi$, the format of Eq.(28) is preserved, but with non-integer coefficients. Thus, the use of non-orthogonal step angles involves no extra mathematical burden. A fivestep scheme was chosen here rather than the conventional four-step method so that a substantial fraction of a wavelength is covered even for steps less than $90^{\circ}$. This helps maintain phase evaluation accuracy or non-orthogonal steps.

\subsection{Filtering and Phase Unwrapping}

The relative phase change $\Delta \phi$ obtained with the arctangent function is called a wrapped phase map because the phase values are wrapped within the range $[-\pi,+\pi]$ as shown in Figure 15 , with modulo $2 \pi$ repetitions for angles outside that range. This is due to the repetitive nature of the arctangent function. To obtain a smooth phase map from the sawtooth pattern, the wrapped phase map is unwrapped to remove the $2 \pi$ phase discontinuities. Only a noiseless sawtooth image can be unwrapped effectively. The noise of the originally generated phase map needs to be filtered. This noise could cause local phase errors of $2 \pi$ or more such damaging the data analysis. 


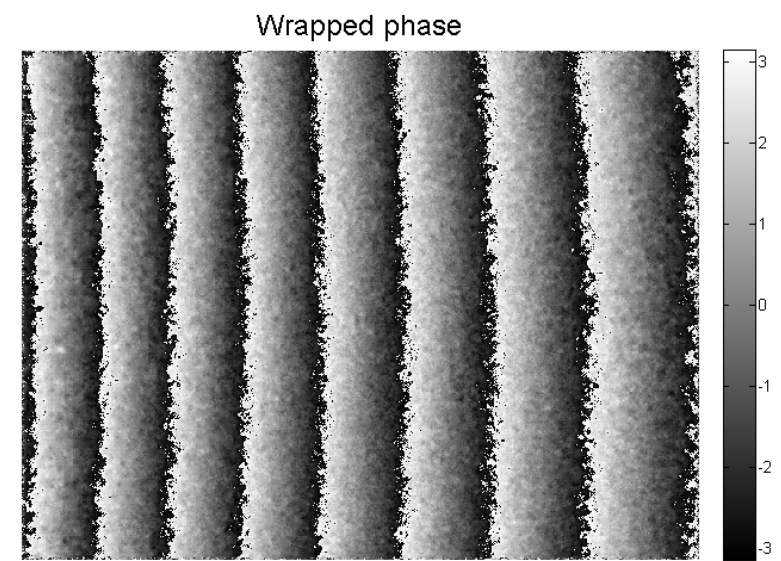

(a)

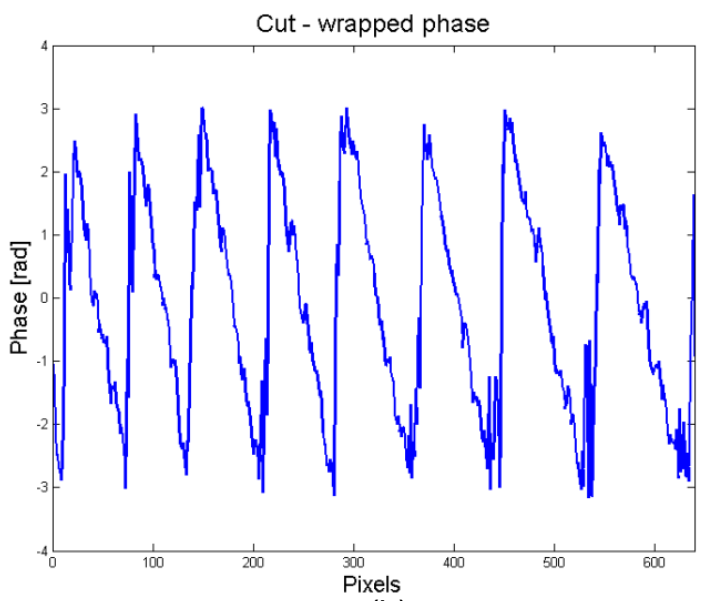

(b)

Figure 15 - (a) fringe pattern (b) sawtooth wrapped phase distribution.

Before applying any unwrapping method, the wrapped phase map must be filtered. An and Schajer described a selective filtering approach [90] where defective pixels are identified according to various measures, and have their phase values interpolated from the adjacent good pixels. In this way, the defective pixels are "repaired" while keeping the adjacent good pixels intact.

After being filtered, the wrapped phase map can be finally unwrapped to remove the $2 \pi$-jumps. The unwrapping process restores the "true" value of the relative phase change as illustrated in Figure 16. As long as the boundary conditions remain unknown, the measured phase is a relative phase. Conventional unwrapping procedures do not result in the absolute phase. With conventional phase unwrapping algorithms, continuous phase maps provide only relative phase values because the absolute phase of the starting point of the unwrapping process cannot be determined [92]. The absolute phase could be extracted using the two-wavelength method. The final unwrapped phase map is used to determine the surface displacements according to the geometrical configuration of the interferometer.

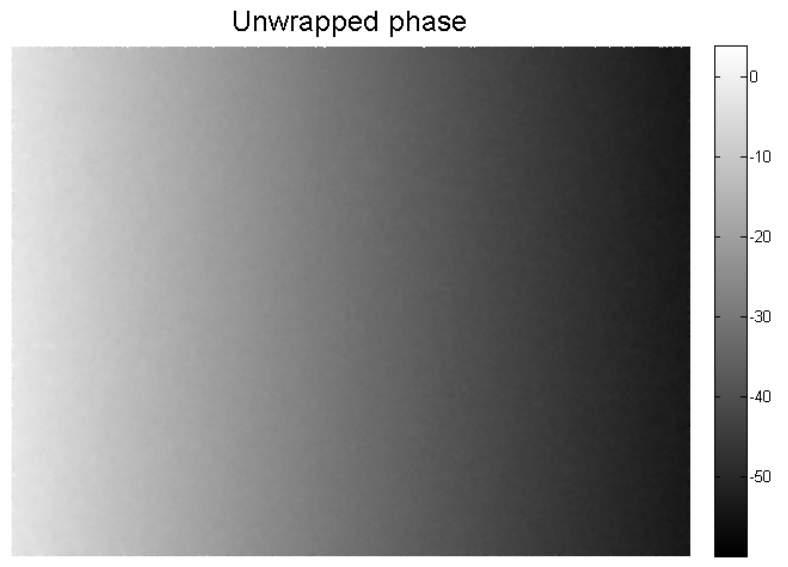

(a)

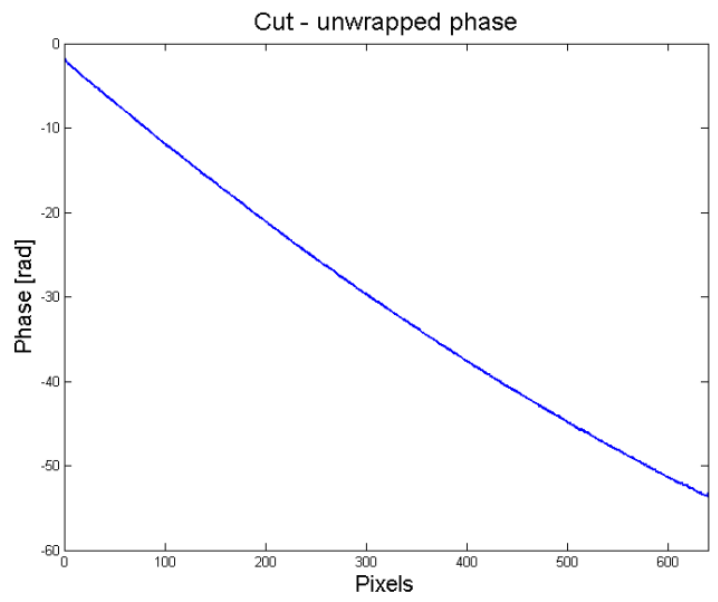

(b)

Figure 16 - (a) unwrapped phase map (b) profile of the unwrapped phase distribution. 


\section{Chapter 4. Shearography}

\subsection{A Typical Shearography Optical Setup}

Figure 17 shows an example ESPI arrangement using a modified Michelson interferometer. This is a common setup for doing shearography $[4,6]$. In this arrangement, a camera views through the interferometer an object illuminated by coherent monochromatic light source. The configuration of the beam splitter and mirrors provides two alternative optical paths. If the components were exactly aligned, the two paths would recombine to image the same points on the object. However, a deliberately induced tilt in one of the mirrors causes a lateral shift in one path such that the camera views two slightly displaced images. In this way, each pixel on the camera receives light from two distinct points on the illuminated object, separated by a distance $\delta x$ called shear distance. The light from the pairs of points interfere on the camera pixels to produce the measured speckle pattern. For this optical arrangement the measured phase map corresponds to the relative length change of the two optical paths through the interferometer. This quantity approximately corresponds to the out-of-plane surface slope in the direction of the image shift.

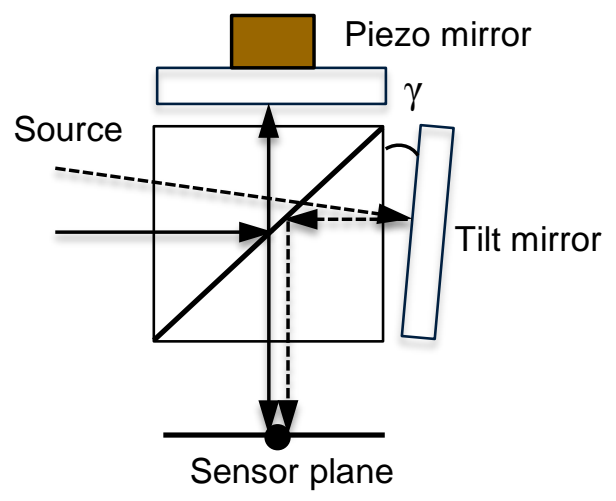

Figure 17 - Example ESPI arrangement for doing shearography.

\subsubsection{Relationship of Fringes to the Optical Phase Change}

A shearographic arrangement with shear adjusted according to the x-direction and the source of laser light lying on the $x-z$ plane measures two components of the surface deformation. The interferometer has no sensitivity in the y-direction. As shown in Figure 18, the optical path length of the light which illuminates the points $P_{1}$ and $P_{2}$, becoming $P_{1}{ }^{\prime}$ and $P_{2}{ }^{\prime}$ after deformation, is affected during deformation of the test object. For an out-of-plane deformation, the change in the optical path length from the laser source to the object surface can be expressed as: 


$$
\Delta L_{\text {out-of-plane,laser }}=\cos \theta \delta x \frac{\partial w}{\partial x}
$$

and from the object surface to the sensor plane the change in the optical path length is:

$$
\Delta L_{\text {out }-o f-p l a n e, \text { sensor }}=\delta x \frac{\partial w}{\partial x}
$$

Figure 18(b) shows an in-plane deformation where the optical path length of the light is only affected from the laser source to the object surface and is depicted as:

$$
\Delta L_{\text {in-plane,laser }}=\sin \theta \delta x \frac{\partial u}{\partial x}
$$

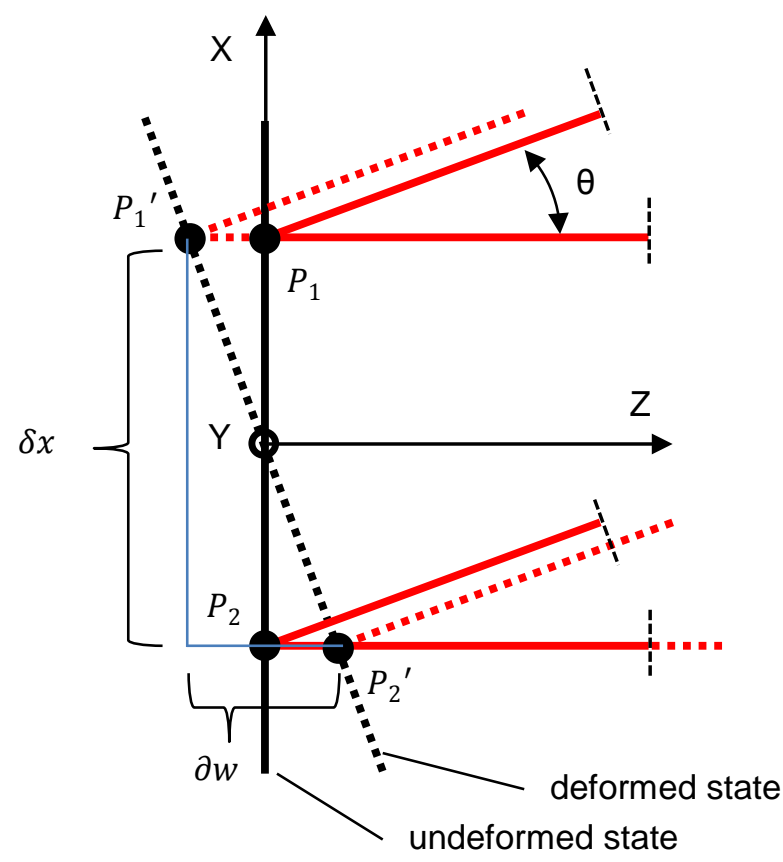

(a)

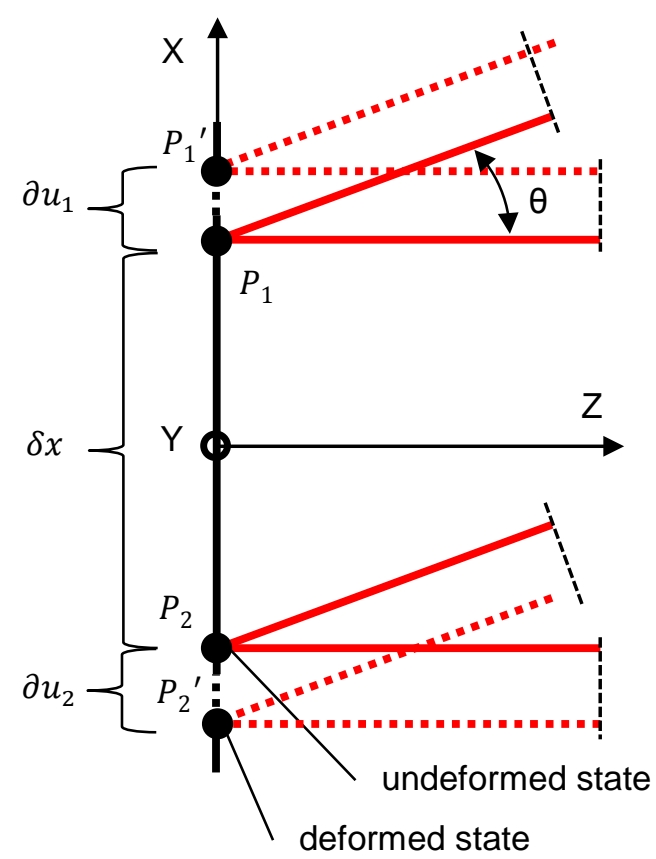

(b)

Figure 18 - Combination of deformation (a) out-of-plane deformation (b) in-plane deformation.

In a more general case, both deformation types are combined and the three different changes in optical path length are summed. Thus the relative phase change of interference pattern due to surface deformation $\Delta \varphi$ with shear in $\mathrm{x}$-direction can be represented by:

$$
\Delta \varphi_{x}=\frac{2 \pi}{\lambda}\left[\sin \theta_{x z} \frac{\partial u}{\partial x}+\left(1+\cos \theta_{x z}\right) \frac{\partial w}{\partial x}\right] \delta x
$$




\subsubsection{Out-of-Plane Sensitive Shearography System}

Figure 19 shows the optical setup for measuring mainly out-of-plane surface displacement derivatives. This setup is widely used in non-destructive testing because it reveals flaws in composite materials by detecting surface deformation-induced anomalies. In general, the in-plane strain term $\partial u / \partial x$ in Eq. (33) is very small compared with the out-of-plane rotation term $\partial w / \partial x$ and can typically be neglected. Under this condition Eq. (33) reduces to:

$$
\Delta \varphi_{x}=\frac{2 \pi}{\lambda}\left[\left(1+\cos \theta_{x z}\right) \frac{\partial w}{\partial x}\right] \delta x
$$

When the illumination angle is $0^{\circ}$ or small enough that the cosine term can be neglected, the relative phase change obtained with shear in $\mathrm{x}$-direction becomes:

$$
\Delta \varphi_{x}=\frac{4 \pi}{\lambda} \frac{\partial w}{\partial x} \delta x
$$

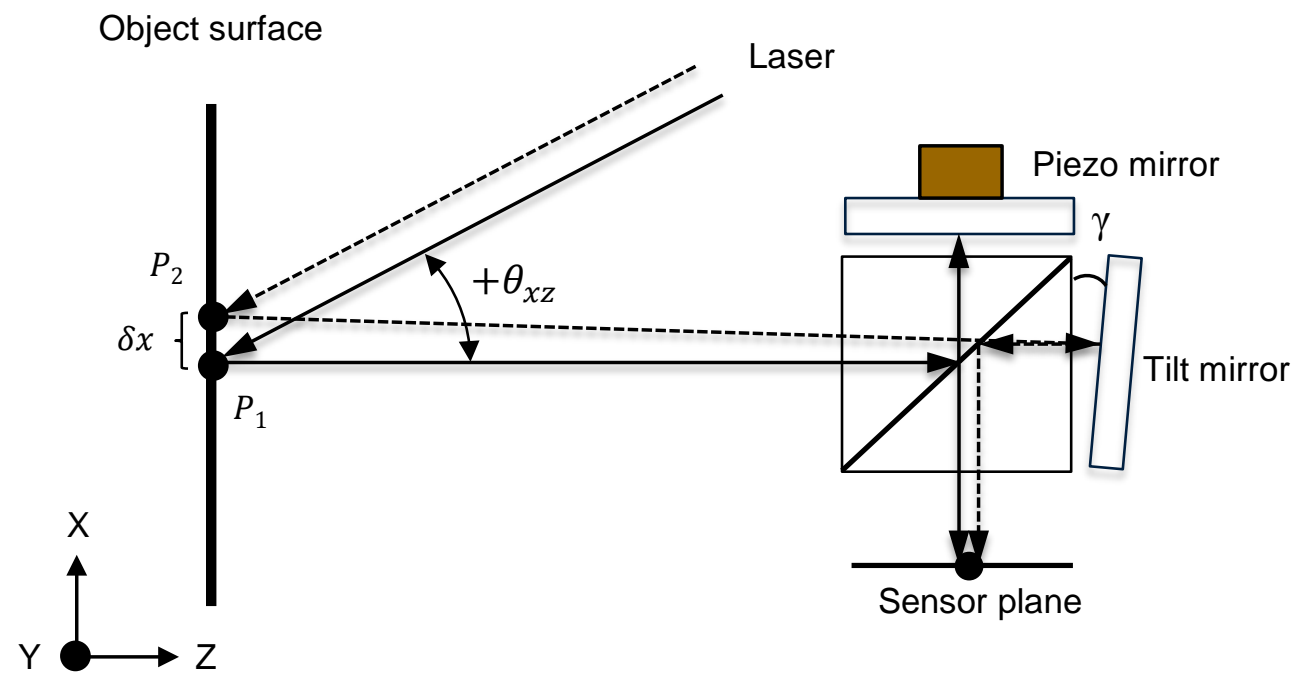

Figure 19 - Optical configuration of an out-of-plane shearographic system.

\subsubsection{In-Plane Sensitive Shearography System}

A dual-illumination system is required to extract the in-plane strain component from the shearographic equations. Figure 20 shows an in-plane shearographic system, where the object surface is alternately illuminated by two collimated monochromatic laser beams. The laser light sources are arranged symmetrically according the viewing direction and are lying on the $x-z$ plane. 


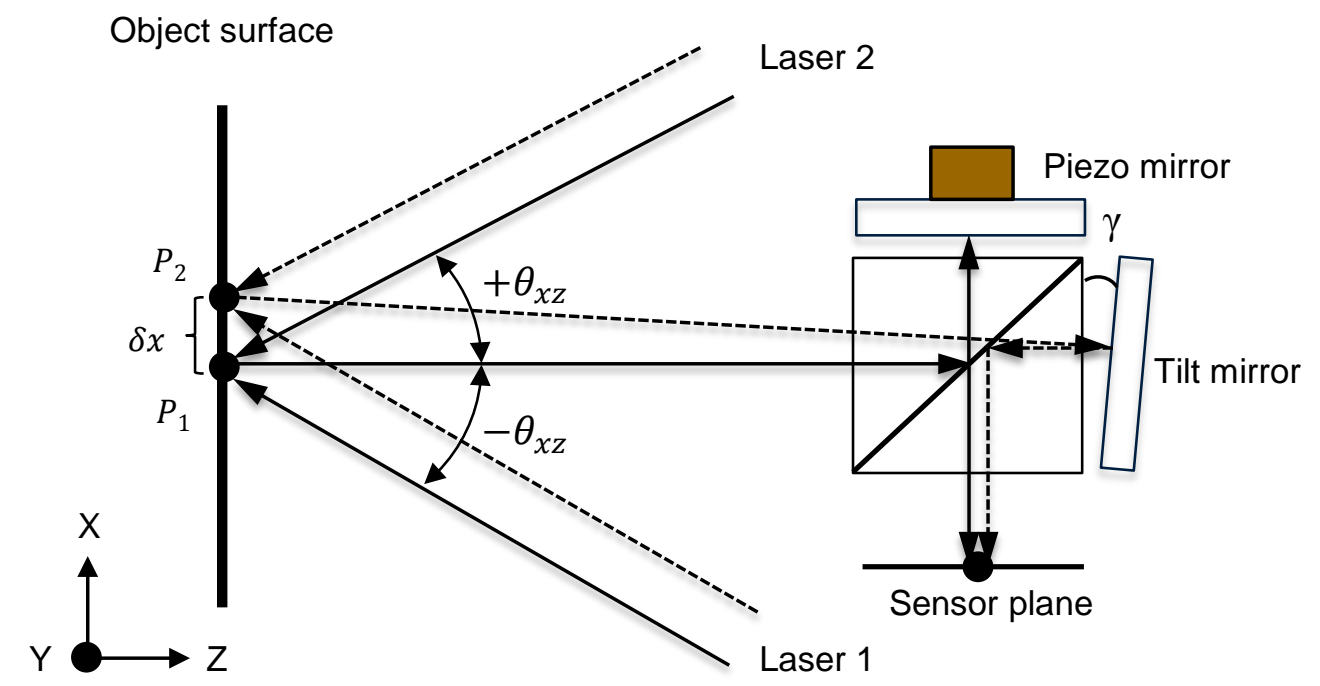

Figure 20 - Optical configuration of an in-plane shearographic system.

When the illumination angles $+\theta$ and $-\theta$ lie in the $x-z$ plane and the shear is in the $x$-direction, the relative phase change for the first source of light becomes:

$$
\Delta \varphi_{x,+\theta}=\frac{2 \pi}{\lambda}\left\{\sin \left(+\theta_{x z}\right) \frac{\partial u}{\partial x}+\left[1+\cos \left(+\theta_{x z}\right)\right] \frac{\partial w}{\partial x}\right\} \delta x
$$

For the second source of light being at a negative angle from the z-axis and taking into account the sign of this angle, with $\sin (-\theta)=-\sin (\theta)$ and $\cos (-\theta)=\cos (\theta)$, the relative phase change is:

$$
\Delta \varphi_{x,-\theta}=\frac{2 \pi}{\lambda}\left\{-\sin \left(\theta_{x z}\right) \frac{\partial u}{\partial x}+\left[1+\cos \left(\theta_{x z}\right)\right] \frac{\partial w}{\partial x}\right\} \delta x
$$

In order to get the in-plane component, the out-of-plane component needs to be removed. This can be achieved by subtracting the phase $\Delta \varphi_{x,+\theta}$ and $\Delta \varphi_{x,-\theta}$. The in-plane surface strain can then be isolated as following:

$$
\Delta \varphi_{x,+\theta}-\Delta \varphi_{x,-\theta}=\frac{4 \pi \delta x}{\lambda} \sin \theta_{x z} \frac{\partial u}{\partial x}
$$

By summing both phases, the out-of-plane slope component can also be isolated:

$$
\Delta \varphi_{x,+\theta}+\Delta \varphi_{x,-\theta}=\frac{4 \pi \delta x}{\lambda}\left(1+\cos \theta_{x z}\right) \frac{\partial w}{\partial x}
$$

The in-plane strain can be measured in all directions by adjusting the orientation of the collimated light sources. Two light sources of different wavelengths and a RGB color camera enable simultaneous measurements. With three different illumination orientations, it should be possible to evaluate strain in all 
three measurement directions, and by extension, in all in-plane directions on the measured surface. Effectively, the combined measurements would provide an optical strain gauge rosette at every pixel within the optical field.

In-plane strain measurements have already been demonstrated and the technique works well for test object put under tension or compression. If the deformation contains not only in-plane components but also out-of-plane components, small imperfections in the arrangement could lead to dramatic errors in the in-plain strain measurement. When using shearography to measure surface strain, it is useful to consider the relative size of the surface strain and the surface rotation. These two must be separated to identify strain. In Figure 21 a cantilever beam is used as a model to illustrate this problem:

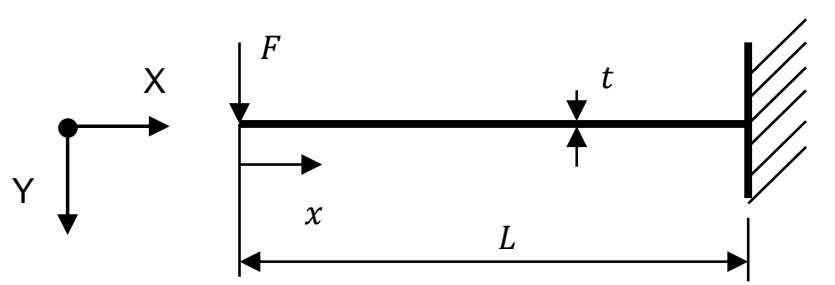

Figure 21 - Cantilever beam in bending.

According to the beam theory, the slope is:

$$
\frac{d^{2} y}{d x^{2}}=\frac{M}{E I}=\frac{-F x}{E I} \rightarrow \frac{d y}{d x}=\frac{F}{E I}\left(L^{2}-x^{2}\right)
$$

The surface strain is:

$$
\varepsilon_{x}=\frac{-M(t / 2)}{E I}=\frac{F t x}{2 E I}
$$

The ratio between the slope and the strain can be calculated as:

$$
\frac{\left(\frac{d y}{d x}\right)}{\varepsilon_{x}}=\frac{L^{2}-x^{2}}{t x}
$$

Table 1 shows clearly that the surface slope is almost always much greater than the surface strain for typical bending cases for which $t \ll L$. Therefore, shearography is likely useful for strain measurements with in-plane loadings only. 
Table 1 - Slope/strain ratio for a cantilever beam with $2 \mathrm{~mm}$ thickness and 200mm length.

\begin{tabular}{|c|c|c|}
\hline$\frac{x}{L}$ & ratio $/(L / t)$ & $\begin{array}{c}\text { Ratio for } \\
L / t=100\end{array}$ \\
\hline 0.5 & & 150 \\
\hline 0.6 & 1.50 & 106 \\
\hline 0.7 & 1.06 & 73 \\
\hline 0.8 & 0.73 & 45 \\
\hline 0.9 & 0.45 & 21 \\
\hline
\end{tabular}

\subsection{Two-Axis Shearography}

The shearography technique in Figure 17 measures the surface slope in the image shearing direction determined by the direction of the mirror tilt used. If measurement of a surface slope in a different direction is required, either the mirror tilt would have to be changed and a further measurement made, or else a second interferometer system containing the desired mirror tilt would have to be used in parallel. Both options are awkward.

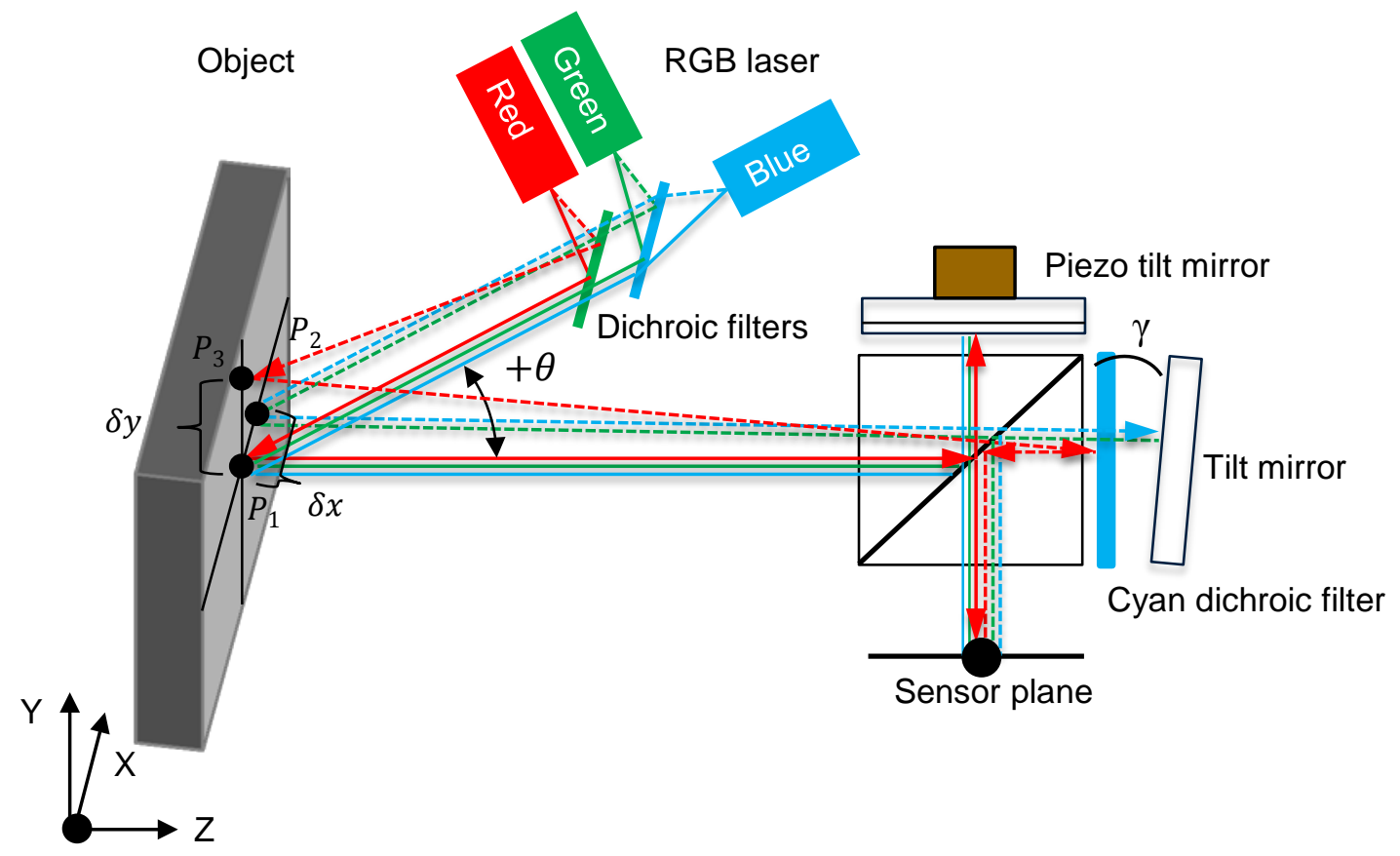

Figure 22 - Optical configuration of a two-axis shearographic system.

Figure 22 illustrates a novel shearography interferometer developed here that enables simultaneous interferometry in two image shearing directions. The interferometer uses a combined RGB (Red, Green, 
and Blue) laser to illuminate the measured surface with coherent light with three distinct wavelengths. Two dichroic filters, disposed at $45^{\circ}$, combine the three colored beam in one beam of light, which is then collimated with a plano-convex lens. The RGB laser works like a single source with the advantage of generating three different wavelengths of light and it removes the complications arising from the use of multiple angles of illumination. The interferometer in Figure 22 differs from the one in Figure 17 by the addition of a dichroic filter between the beamsplitter cube and the tilt mirror. The dichroic filter has the property of passing blue and green light and reflecting red light. Thus, the tilt mirror reflects the green and blue light and the dichroic filter reflects the red light. If the tilt mirror and the dichroic filters are appropriately aligned, the green/blue light can be sheared in one direction, say $x$, and the red light sheared in a different direction, say $y$. Thus, if interferometry measurements are made using a color camera, surface slope measurements in both the $x$ and $y$ directions can be made simultaneously.

Of a double shearing interferometer using multiple coherent light sources of different wavelengths, two modified Michelson interferometers are arranged in a single interferometer, one with a mirror tilted in the $x$-direction on the specimen, and the second with a mirror tilted in the y-direction. Illumination by green or blue light will display only the shearing in the $x$-direction, while illumination by red light will display only shearing in the $y$-direction. The surface slope measured according the $x$-direction and its respective shearing distance $\delta x$ with green or blue illumination can be expressed as:

$$
\Delta \varphi_{x, \text { green }}=\frac{2 \pi}{\lambda}\left[\sin \theta_{x z} \frac{\partial u}{\partial x}+\left(1+\cos \theta_{x z}\right) \frac{\partial w}{\partial x}\right] \delta x
$$

While the surface slope measured according the $y$-direction and its respective shearing distance $\delta y$ with red illumination is:

$$
\Delta \varphi_{y, r e d}=\frac{2 \pi}{\lambda}\left[\sin \theta_{x z} \frac{\partial u}{\partial y}+\left(1+\cos \theta_{x z}\right) \frac{\partial w}{\partial y}\right] \delta y
$$

Having two interferometers may cause additional problem for referencing the two pairs of sheared images, but with that arrangement, the sheared images share the same reference. The cyan dichroic filter remains fixed while the two tilt mirrors need to be adjusted in function of the desired shear orientation and amplitude. The piezzo-tilt mirror, which affects both pairs of images, is first brought into position followed by the tilt mirror.

The innovative "two interferometers in one" concept is made possible with the use of a dichroic filter. A dichroic filter is an accurate color filter coated with multi-layer thin-films and it operates by the principles of interference. This type of filter transmits selectively certain wavelengths of light while reflecting the other frequencies, rather than absorbing them. Coatings on dichroic filters can be seen in terms of many FabryPerot cavities as illustrated in Figure 23. Multiple layers of dielectric materials with different indices of refraction cause multiple internal reflections when a beam of light penetrates them. Those reflections, 
originating from the mirrored surfaces, interfere constructively or destructively at certain wavelengths thus filtering the incident light. Dichroic filters can be designed and produced to nearly any specification of band passes and center wavelengths, except that the production costs could be very high for nonstandard filters.

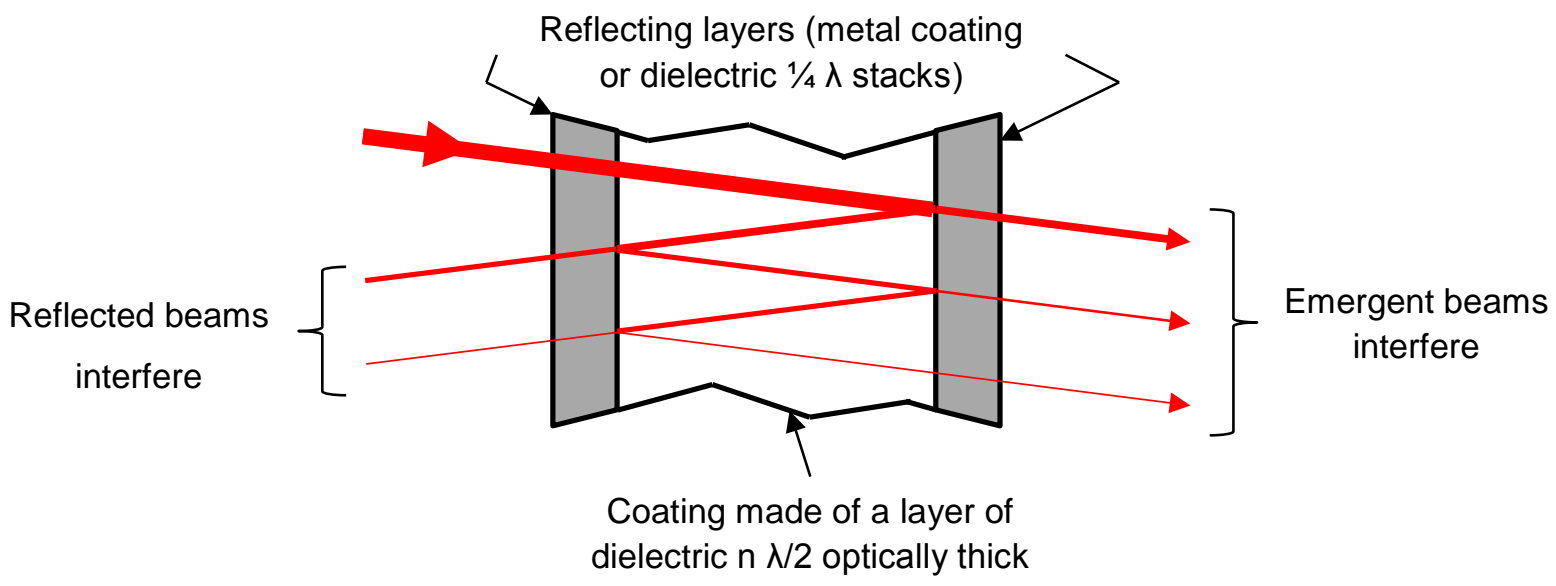

Figure 23 - Single Fabry-Perot cavity.

This concept is developed here, where the target object is simultaneously illuminated by three coherent laser sources of different wavelengths, corresponding to red, green and blue. The light passes from the object through the double interferometer to a color camera that is able to record each color separately. This color separation allows separate measurement of the two shearing directions and hence separate identification of the surface slopes. Green and blue are used redundantly to measure the same surface slope to identify the absolute phase using the "Two-Wavelength" method.

\subsection{Shear Distance}

As the shown in Equation (33), the system sensitivity is a function of the wavelength of the laser, the angle of illumination, as well as the shear distance. Lee et al. wrote: "In shearography, shear distance is the most important parameter to control measurement performances" [93]. They investigated theoretically and experimentally the relationship between shear distance and performance issues such as the immeasurable zone in the target with discontinuity, signal-to-noise ratio, sensitivity and shear distortion. The system sensitivity can be enhanced by increasing the shear distance. However, it implies two major drawbacks which are an increase in signal-to-noise ratio (SNR) and a loss of spatial resolution.

The shear distance needs to be accurately measured. The grid method allows for displacement measurements and was successfully applied for measuring the shear distance [94]. The grid method is a 
geometrical method where the wavelength of light is not involved. A grid, generated by computer, is printed and taped on the front surface of the test specimen. The shear distance is calculated from the displacement of grid lines caused by the tilt introduced in one of the mirror of the modified Michelson interferometer. Lee et al. measured shear distances and their respective distortions using a grid with 2.5 $\mathrm{mm}$ pitch [93], they concluded that the geometrical shear distortion in the Michelson interferometer was negligible both experimentally and theoretically for the general shear distances less than $5 \mathrm{~mm}$.

NG T.W. used Digital Image Correlation (DIC) to measure the amount of shear in shearography [95]. The technique does not require a tape target and allows the shear distance to be measured, regardless of the nature and location of the object. This method involves two general steps, where the first step consists in calibrating the object surface distance to picture element (pixel) spacing ratio and the second, in determining the amount of shear. To measure the shear distance two pattern images, such as a speckle pattern printed on the object surface, are captured. Each image is recorded with one of the mirrors inside the modified Michelson interferometer blocked with an opaque piece of paper. A 2D digital image correlation algorithm is used to generate a map of shear distances.

The applied shear varies across the object surface when measuring curved object due to its curvature. Groves et al. measured both the shape and the in-plane and out-of-plane displacement gradients of a highly curved object [96]. They corrected the error due to the dependence of the applied shear upon the local surface slope using knowledge of the slope of the object.

\subsubsection{Correction for Non-Uniform Shear}

In typical measurements, the shearing distance is in the range $5-10 \%$ of the image width. This is a modest fraction of the image size, so aberrations due to minor deviations in the flatness of the mirrors and dichroic filters can become significant. Such flatness deviations cause small variations in the shearing amount across the measured image and can cause noticeable discrepancies when high-accuracy measurements are pursued.

The surface non-uniformity of a mirror can be identified as local and global as shown in Figure 24. The local flatness, specified as a fraction of a wavelength over a certain length, is characterized by peaks and valleys while the global flatness describes distortions over the entire mirror. The global flatness has a bigger effect on the image distortion and should be considered prior to the local flatness. As stated by Lee et al. [93], when using optical-grade mirrors the distortion is negligible for doing shearography. 


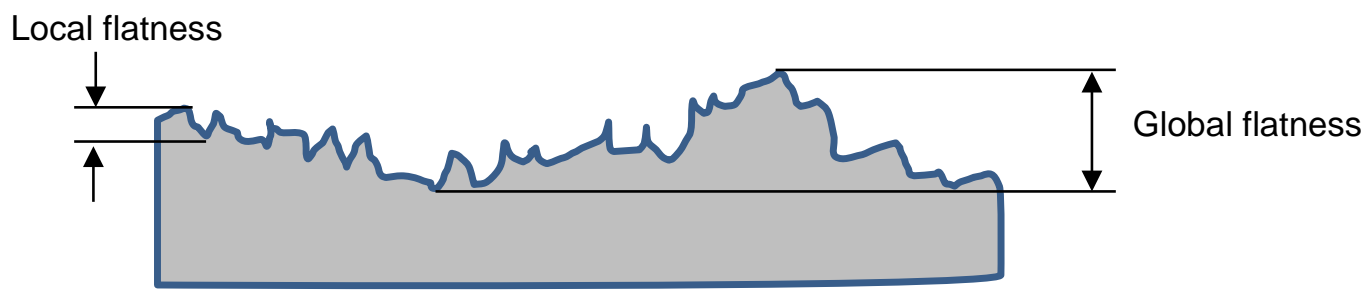

Figure 24 - Surface non-uniformity of a mirror.

The cyan dichroic filter used in the present work was chosen for its good transmission/reflection characteristics, these greatly help in producing high quality interference fringes. However, this style is filter could only be obtained in a $0.7 \mathrm{~mm}$ thickness format, which is difficult to mount properly without deformation. The non-flatness of the dichroic filter affects the reflected red colored signal but not the blue and green colors since blue and green are passing through. Fortunately, the image distortion originating from this non-flatness can be corrected mathematically.

The mathematical correction for non-uniform shear not only corrects for mirror non-flatness but also for mirror misalignment. When adjusting the shear, the piezo-tilt mirror, working in pair with the dichroic filter, is first moved to get the desired shear distance in y-direction. Then the tilt-mirror, operating with the piezotilt mirror, is adjusted for a shear along $x$-direction. The first interferometer has supposedly pure shear in $y$-direction as well as the second interferometer in x-direction. The mirrors are mounted on three-point optical brackets with fine screws allowing for easy adjustment. Even with fine adjustment, it is almost impossible to position the mirrors to get the two pair images perfectly shifted in their respective direction.

An example of measured shears for a two-axis shearography arrangement is presented in Figure 25. The open source DIC software Ncorr [97] has been used to get the different shear maps. The tilt mirror was oriented thus the red signal was sheared along the $y$-axis and the piezo-tilt mirror was moved to get the green-blue signals sheared along the x-axis. In Figure 25 (a) and (b), the shear non-uniformity for the green-blue signals is around 1.5 pixels in the x-direction and 1.0 pixels in the y-direction. Figure 25 (c) and (d) show clearly that the cyan dichroic is not flat. The non-flatness and filter misalignment produce a shear non-uniformity around 7.9 pixels in the x-direction and 3.4 pixel in the $y$-direction for the red signal. 


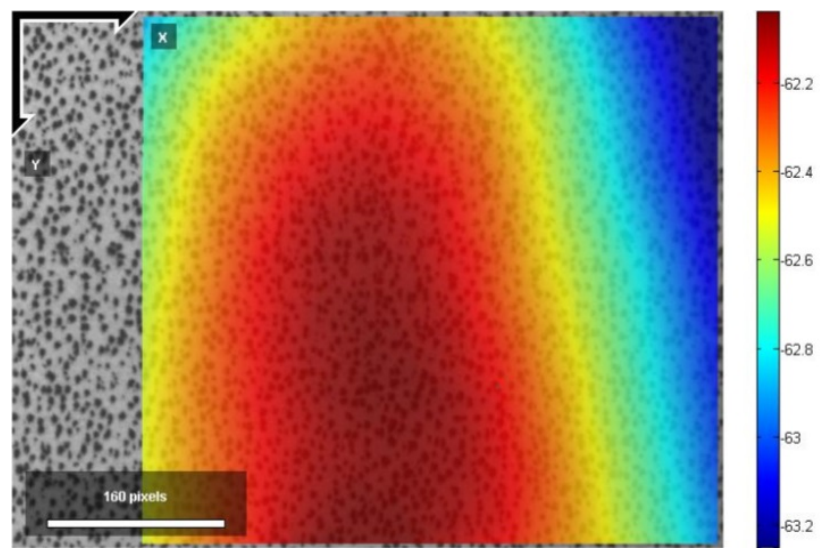

(a)

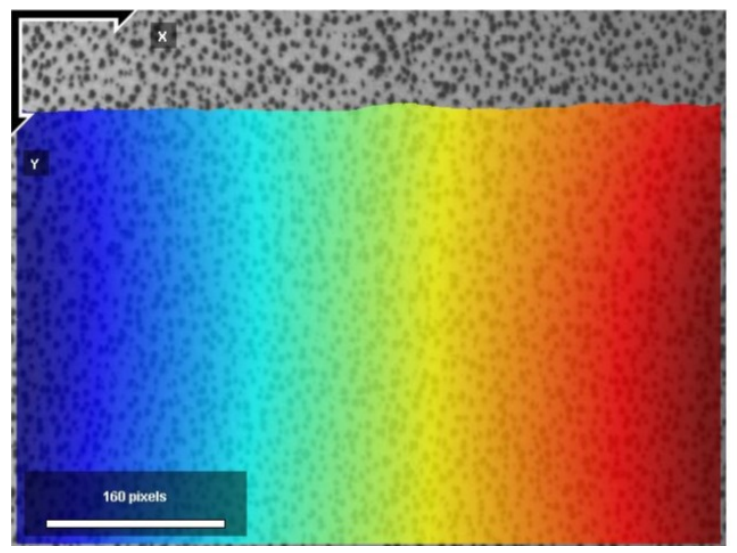

(c)

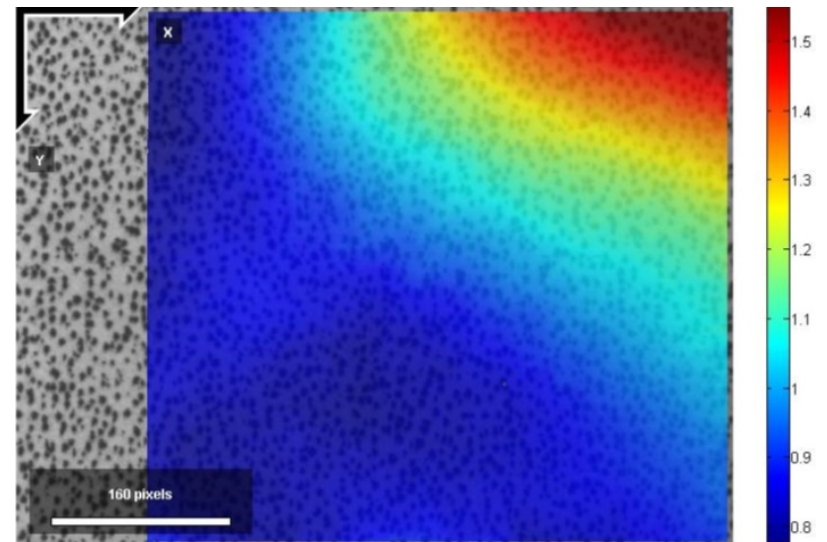

(b)

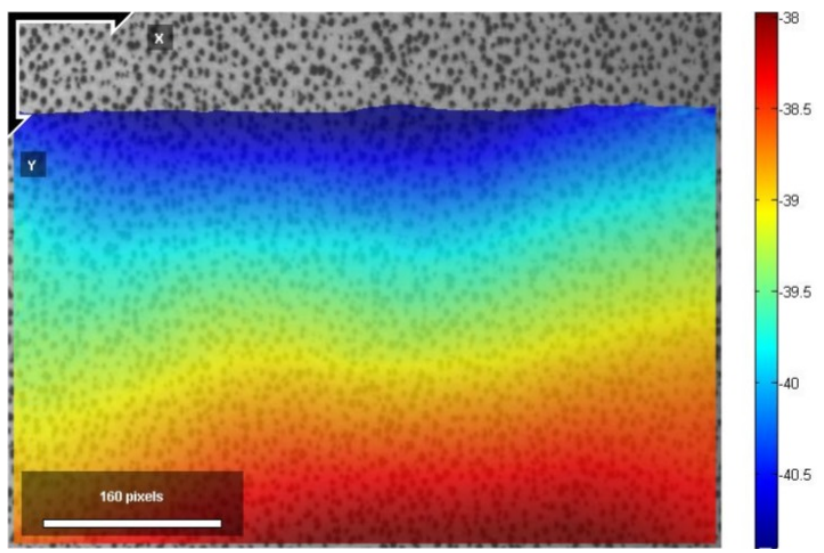

(d)

Figure 25 - Shear maps (a) $x$-shear map in $x$-direction (b) $x$-shear map in $y$-direction (c) $y$-shear map in $x$ direction (d) $y$-shear map in y-direction.

Working with two colors, green (1-color) and red (2-color) for example, the measured phase, with green color and uniform shear in $\mathrm{x}$-direction over the entire image, should be perfect. Measured with red color and uniform shear in $y$-direction, the phase should also be ideal. In reality, neither shear is purely in the axial direction nor uniform. The correction estimates the ideal case from non-ideal measurements. With $x-$ axis being 1-direction and y-axis 2-direction, the measured phases can be expressed as:

$$
\varphi_{1}=\varphi_{11}+\varphi_{12} \text { and } \varphi_{2}=\varphi_{21}+\varphi_{22}
$$

with $\varphi_{1}$ being the measured phase in red light, $\varphi_{2}$ being the measured phase in green light, and $\varphi_{i j}$ being the component of $\varphi_{i}$ in j-direction.

Using $S_{1}$ as the ideal uniform shear in 1-direction with zero shear in 2-direction, $S_{2}$ as the ideal uniform shear in 2-direction with zero shear in 1-direction, $\Delta_{11}$ as the actual 1-shear in red light (expected $\cong S_{1}$ ), 
$\Delta_{22}$ as the actual 2-shear in blue light (expected $\cong S_{2}$ ), and assuming linear surface rotation the corrected phases can be written:

$$
\Phi_{1} \cong \frac{s_{1}}{\Delta_{11}} \varphi_{11} \text { and } \Phi_{2} \cong \frac{s_{2}}{\Delta_{22}} \varphi_{22}
$$

Inverting:

$$
\varphi_{11} \cong \frac{\Delta_{11}}{S_{1}} \Phi_{1} \text { and } \varphi_{22} \cong \frac{\Delta_{22}}{S_{2}} \Phi_{2}
$$

Estimating and taking into account for multiple wavelengths:

$$
\varphi_{21} \cong \frac{\Delta_{21}}{S_{1}} \frac{\lambda_{1}}{\lambda_{2}} \Phi_{1} \text { and } \varphi_{12} \cong \frac{\Delta_{12}}{S_{2}} \frac{\lambda_{2}}{\lambda_{1}} \Phi_{2}
$$

Assembling:

$$
\varphi_{1}=\varphi_{11}+\varphi_{12} \cong \frac{\Delta_{11}}{S_{1}} \Phi_{1}+\frac{\Delta_{12}}{S_{2}} \frac{\lambda_{2}}{\lambda_{1}} \Phi_{2} \text { and } \varphi_{2}=\varphi_{21}+\varphi_{22} \cong \frac{\Delta_{21}}{S_{1}} \frac{\lambda_{1}}{\lambda_{2}} \Phi_{1}+\frac{\Delta_{22}}{S_{2}} \Phi_{2}
$$

In matrix form:

$$
\left[\begin{array}{cc}
\frac{\Delta_{11}}{S_{1}} & \frac{\Delta_{12}}{S_{2}} \frac{\lambda_{2}}{\lambda_{1}} \\
\frac{\Delta_{21}}{S_{1}} \frac{\lambda_{1}}{\lambda_{2}} & \frac{\Delta_{22}}{S_{2}}
\end{array}\right]\left[\begin{array}{l}
\Phi_{1} \\
\Phi_{2}
\end{array}\right] \cong\left[\begin{array}{l}
\varphi_{1} \\
\varphi_{2}
\end{array}\right]
$$

The actual shears $\Delta_{i j}$ are measured using DIC, with best-fit interpolations evaluated so that the local shears at each point can be determined. For best estimations, $S_{1}$ and $S_{2}$ are chosen to be the nearest integers to the averages of $\Delta_{11}$ and $\Delta_{22}$ in the measured images. Then, the diagonal matrix terms will be $\cong 1$ and the off-diagonals $\cong 0$. The corrected phases $\Phi_{1}$ and $\Phi_{2}$ will be obtained by solving the matrix equation. The phase correction is made possible because phases are measured in two orthogonal directions. The two phase maps are needed for mutual corrections.

\subsection{Absolute Phase Determination}

Most optical interferometric methods are relative measurements of the phase of light between a reference and a given state. Interference fringes need to be interpreted to yield quantitative data. For complicated situations where no convenient boundary conditions exist, this interpretation requires evaluation of the absolute fringe-order. The number of fringe order $N$ is represented as: 


$$
N=\frac{\varphi}{2 \pi}
$$

Photoelasticity is an optical interferometric method used to determine the stress distribution in birefringent material. When illuminating a birefringent object with monochromatic light, dark and bright fringes will be observed. If white light illumination is used on the same object, black and colored fringes will appear. Black corresponds to the zero-order $N=0$ fringe, while higher order fringes are colored.

Knowledge of the absolute fringe-order is not always necessary. It is possible to obtain quantitative value from interferograms when the boundary conditions and loadings are known with respect to spatial coordinates. Figure 26 shows the measured phases before and after phase unwrapping of a circumferentially clamped membrane loaded on its center. If the sensor encompasses the entire membrane including the clamped edge, it becomes relatively easy to determine zero-order fringe. However, this practice remains limited to this simple case and more sophisticated techniques are needed for handling the complex boundary conditions encountered in industry.

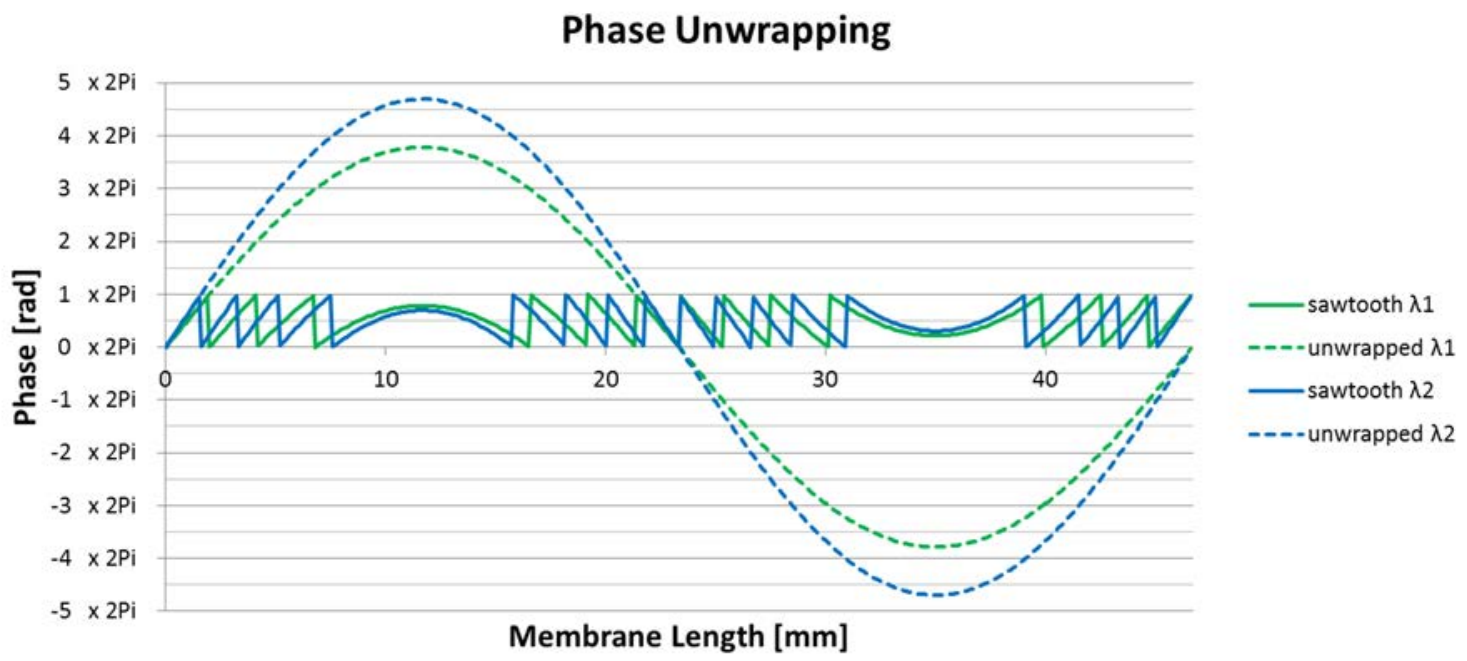

Figure 26 - Wrapped and unwrapped phases of a circular membrane loaded at its center.

The two-wavelength method is applied for absolute phase identification. As described by Steinchen [6], the technique has already been used in holographic interferometry to determine the absolute fringe order without indication of the boundary conditions [95,96]. Two wavelengths $\lambda_{1}$ and $\lambda_{2}$ are involved in the measuring process where $\lambda_{1}>\lambda_{2}$. The wavelength ratio can be written as:

$$
\frac{\lambda_{2}}{\lambda_{1}}=1-\alpha
$$


The measured phase for wavelength $\lambda_{1}$ is $\varphi$ and for $\lambda_{2}$ is $\tau$ :

$$
\frac{\varphi}{\tau}=\frac{\lambda_{2}}{\lambda_{1}}=1-\alpha
$$

The measured phase $\varphi$ and $\tau$ may have multiple $2 \pi$ added offsets, thus the absolute phases can be written as:

$$
\frac{\varphi-2 \pi m}{\tau-2 \pi n}=1-\alpha
$$

where $n$ and $m$ are integers. Rearranging gives:

$$
\frac{\varphi-(1-\alpha) \tau}{2 \pi}=m-n+\alpha n
$$

This result could be used at one pixel, but it will be very noisy. It is better to use the average of many pixels:

$$
s=\frac{\operatorname{average}[\varphi-(1-\alpha) \tau]}{2 \pi}=m-n+\alpha n
$$

$s$ will be a decimal number, such as:

$$
s=k+f
$$

where $k=\operatorname{round}(s)$ is the integer part and $f=s-k$ is the fractional part. The integers $n$ and $m$ can be expressed as:

$$
\begin{gathered}
n=\frac{f}{\alpha} \\
m=k+n
\end{gathered}
$$

To measure absolute phases during experimentations, green $\lambda_{1}=532 \mathrm{~nm}$ and blue $\lambda_{2}=473 \mathrm{~nm}$ laser lights have been used, thus leading to the wavelength ratio $\frac{\lambda_{2}}{\lambda_{1}}=\frac{473}{532}=0.889 \rightarrow \alpha=0.111$. The fractional part is contained in the range $-0.5<f<0.5$. The two-wavelength method is constrained by the wavelength ratio where $n<\frac{1}{0.111}<9$, therefore it should be possible to identify the fringe order within the range $-4.5<n<4.5$. One solution to increase this effective range of calculation is to choose closer wavelengths, but this makes $\alpha$ smaller and thus the evaluation of $f$ becomes more sensitive to measurement noise. 


\subsection{Surface Slopes Summation and Surface Displacement Shape}

For illumination and viewing normal to the object surface, a shearographic interferometer measures the first derivative of the out-of-plane deformation, or slope, of the specimen surface. The slope is a result of surface rotations and is defined by the ratio of the vertical change to the horizontal change between two distinct points on that surface. For an optical arrangement with shear distance adjusted along the $x$ direction, the horizontal change is the shear distance while the vertical change is the out-of-plane surface deformation. As illustrates in Figure 27, the slope corresponds to the ratio of the shear distance $\delta x$ between two points, $\mathrm{P} 1$ and $\mathrm{P} 2$, to the out-of-plane deformation $\delta w$. The shear distance $\delta x$ is known and the out-of-plane deformation $\delta w$ is measured by the shearographic interferometer through the change in optical path length due to surface rotation.
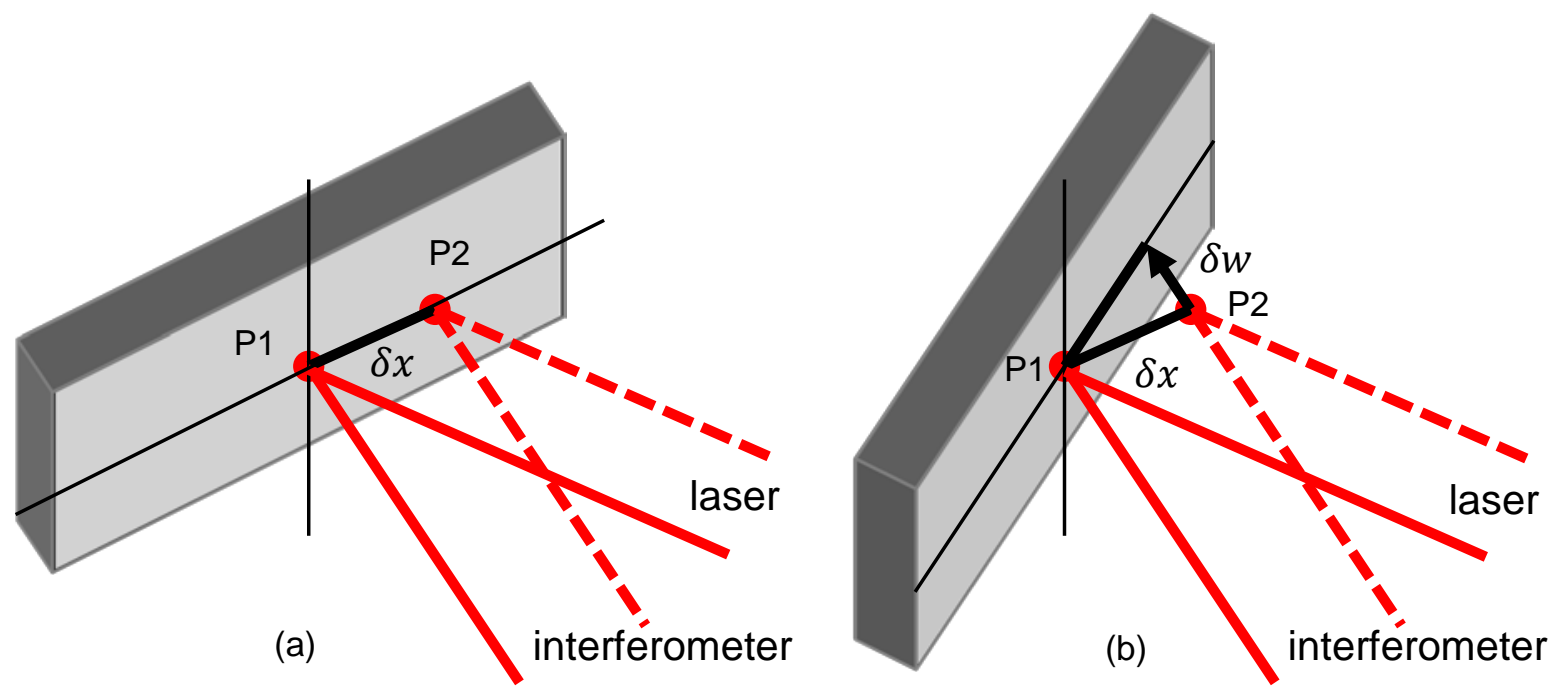

Figure 27 - Change in optical path length due to surface rotation (a) reference state (b) deformed state.

Summation in two directions of the slope yields the deformed shape of the object. The two-axis shearography arrangement provides the two phase maps necessary for calculating the displacement field. Each pixel contains a phase value being proportional to the out-of-plane displacement. This phase value needs to be summed over the shear distance before to be converted into displacement. The basic idea of slope summation is shown in Figure 28. The first value of the first row of the phase map with $x$ shear is interpolated over the shear distance, which corresponds to 3 pixels in that example. The first value of the reconstructed displacement field is chosen to be zero. Then the second value of the first row of the phase map is added to the second displacement value to find the fifth displacement value. The process continue so on over the entire first row and propagates over all rows to form a surface shape. 


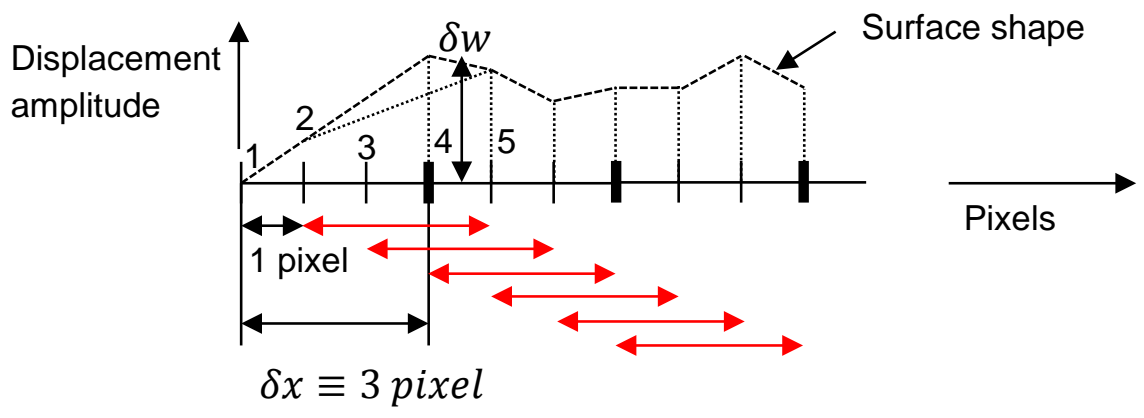

Figure 28 - One direction slope summation.

The two-direction summation works with the same principle. Two phase maps with two orthogonal shears are necessary. The phase is summed horizontally over $\delta x$ while the vertical summation is made over $\delta y$ as illustrated in Figure 29. The summation process is relatively stable. The process starts with the first row and column, which are heavily smoothed to prevent local measurement noise from propagating across the image. The first quadrant is filled using a bilinear interpolation. It is assumed that the phase values at the four corners are known but the bottom right corner is still missing. The fourth corner of the first quadrant can be calculated as:

$$
\begin{gathered}
\Delta \varphi_{r e c}(\delta x, 1)=\Delta \varphi_{x}(1,1) \frac{\operatorname{round}(\delta x)}{\delta x} \\
\Delta \varphi_{r e c}(1, \delta y)=\Delta \varphi_{y}(1,1) \frac{\operatorname{round}(\delta y)}{\delta y} \frac{\lambda_{1}}{\lambda_{2}} \\
\Delta \varphi_{r e c}(\delta x, \delta y)=\left[\Delta \varphi_{r e c}(\delta x, 1)+\Delta \varphi_{r e c}(1, \delta y) \frac{\operatorname{round}(\delta y)}{\delta y}+\Delta \varphi_{r e c}(1, \delta y)\right. \\
\left.+\Delta \varphi_{r e c}(\delta x, 1) \frac{\operatorname{round}(\delta x)}{\delta x}\right] / 2
\end{gathered}
$$

where $\Delta \varphi_{\text {rec }}$ is the reconstructed or summed phase, $\Delta \varphi_{x}(1,1)$ is first top left value of the phase map with $\mathrm{x}$-shear, and $\Delta \varphi_{y}$ is the phase map with $\mathrm{y}$-shear. The second quadrant is calculated two different ways and then averaged. The summation is first achieved horizontally and once the row $\Delta \varphi_{\text {rec }}(n, \delta y)$ is found, the phase values can be vertically and linearly interpolated with the first row. The third quadrant is filled in the same way as for the second quadrant with directions $90^{\circ}$ flipped. The fourth and last quadrant is the largest part of the reconstruction. Each reconstructed phase value originates from vertical and horizontal summation. To get the displacement field, the reconstructed phase map need to be converted with respect to illumination orientation, thus the deformed shape of the object surface can be written:

$$
d w(x, y)=\Delta \varphi_{r e c}(x, y) \frac{\lambda_{1}}{2 \pi\left(1+\cos \theta_{x z}\right)}
$$




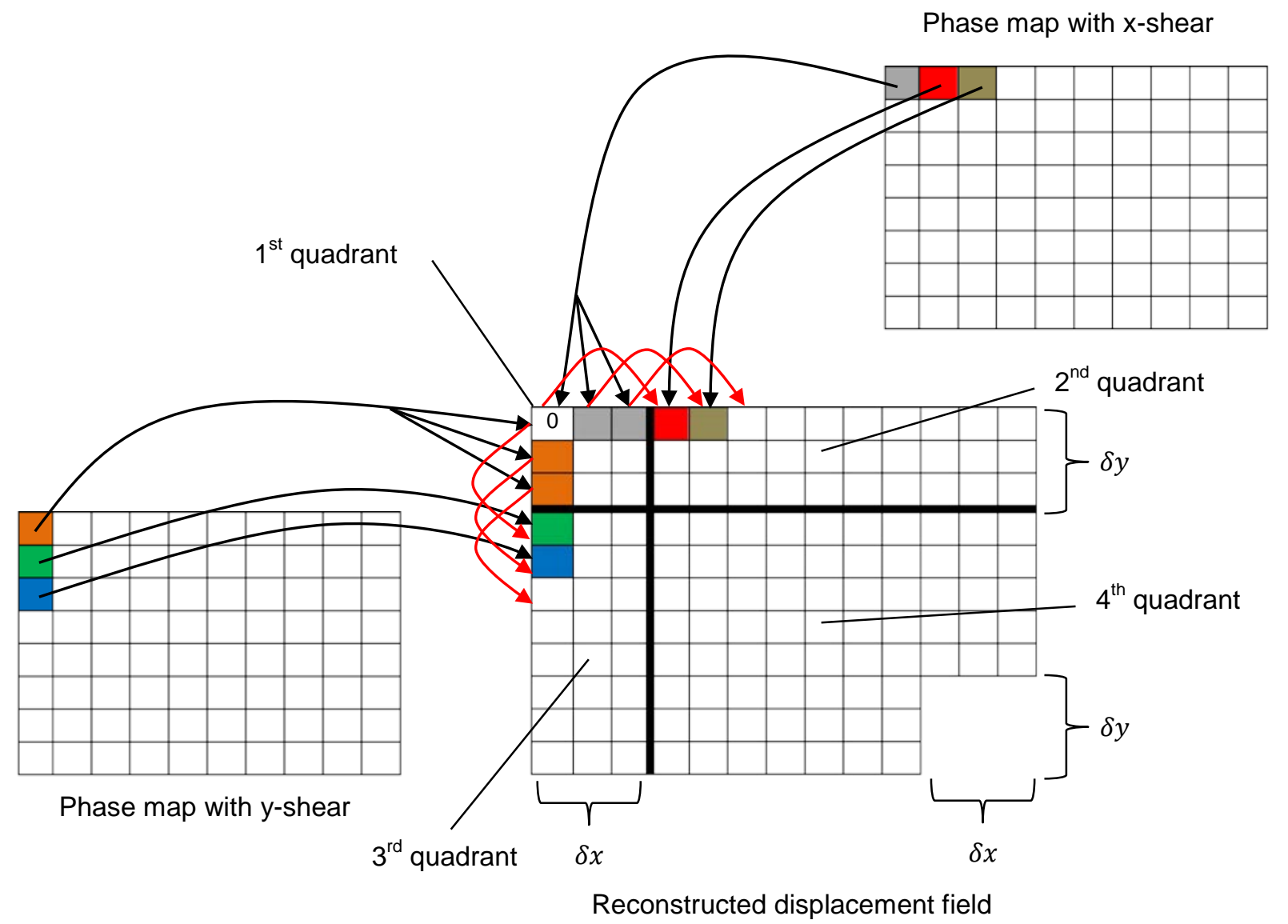

Figure 29 - Schematic of the summation method to retrieve the displacement field.

Stephan Waldner from the Swiss Federal Institute of Technology of Zurich also worked on the phase summation to get the displacement field [82]. The main difference between his method and the method used here is the work done on the starting edges, first to third quadrant, so as to prevent measurement noise from repeatedly propagating across the image. The results demonstrated by Waldner are heavily filtered. Here, the method requires smoothing only in the starting row and column but not within the bulk of the image. This approach avoids excessive smoothing of the resulting summed phase map so as to retain the measurement texture of the two shearography phase maps from which it is derived. Waldner introduced two main advantages with phase summation, or "removing the image doubling" as he called it. Indeed, the two sheared images cause a doubling of the image, causing problems when inspecting regions of interest around features like crack tips, notches, or holes smaller than the shear distance. With shearography, the measured quantity is an approximation to the displacement derivative. The phase summation has the advantage of removing the image doubling and yields a displacement field that in theory is "exact". 


\section{Chapter 5. Experimental Validation}

This chapter presents the results from shearography measurements of an overhanging beam in bending. The use of a color camera and different colored lasers for doing simultaneous ESPI measurements is demonstrated via measurements performed with the novel dual-axis shearographic interferometer described in Chapter 4.2. For validation, the measured data are compared with known deformations of an overhanging beam.

The interferometer was designed to be sensitive, mainly, to out-of-plane displacements, thus an illumination angle of $15.8^{\circ}$ was used when measuring the surface slope of the deformed beam. Two experiments were carried out to validate the theory developed in the previous chapters. A first test was performed to measure the relative slope in two orthogonal directions, the absolute phase in one direction, and the deformed shape of the beam. All measured values include the correction for non-uniform shear. The second test, where the tilt mirror was oriented for a shear along the $\mathrm{x}$-axis while the piezo-tilt mirror was oriented with deliberate $x$-shear and $y$-shear mirror misalignment, shows the effective operation of the correction algorithm.

\subsection{Shearography Experimental Setup}

Figure 30 shows the experimental setup designed and built for doing shearography in two orthogonal axes. The arrangement is composed of four subsystems, which are: a RGB laser source with its collimation system, an overhanging beam, a double-shearographic interferometer, and a camera. All elements are rigidly mounted onto an optical table, which is supported by four air bearings to minimize external sources of vibrations. The major components of the setup are listed in Table 2. All non-standard elements were custom designed and built. 


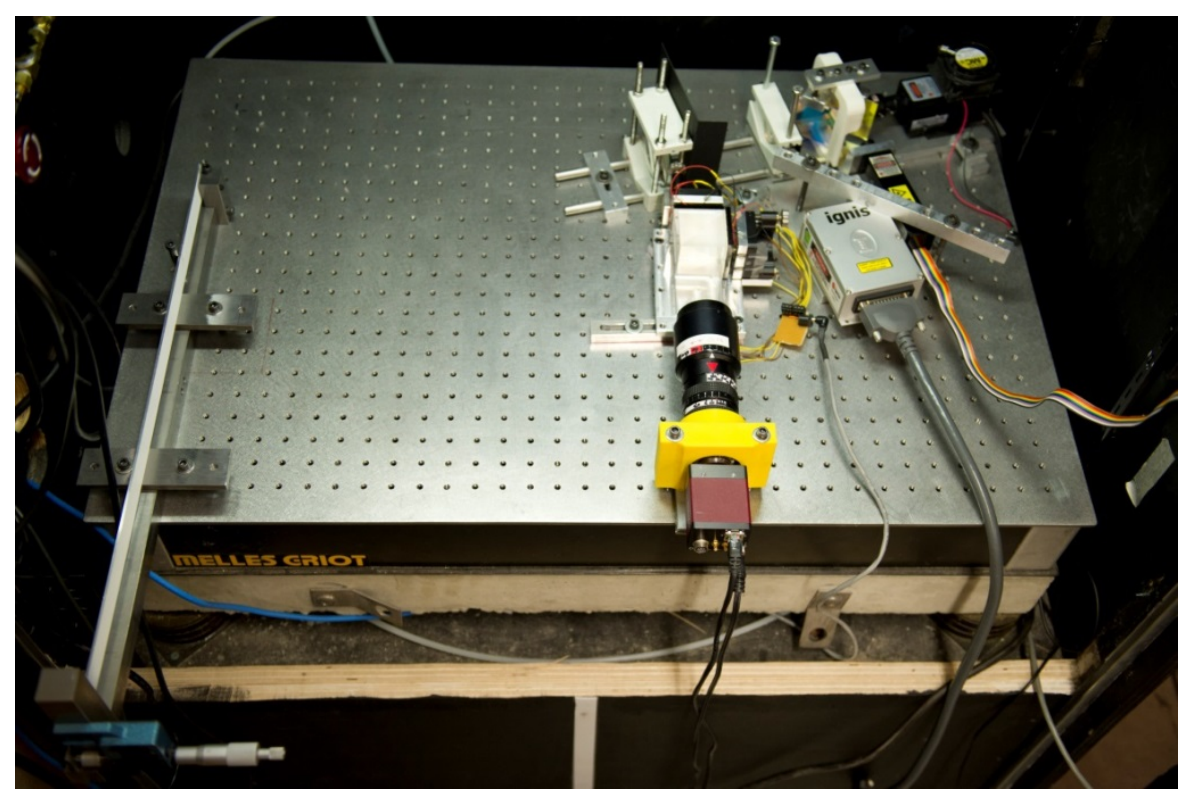

Figure 30 - Experimental setup for doing Two-Axis Shearography to measure surface deformations on an overhanging beam in bending.

Table 2 - List of components used for the experimental setup

\begin{tabular}{|c|c|c|}
\hline Component & Model & Specifications \\
\hline \multicolumn{3}{|l|}{ RGB laser source } \\
\hline Red laser & $\begin{array}{l}\text { Ignis Free Space 660nm SMD6000, } \\
\text { L-IGNIS660FS-SMD6H }\end{array}$ & $660 \mathrm{~nm}$ wavelength, $270-330 \mathrm{~mW}$ \\
\hline Green laser & CrystaLaser GCL-100-S & 532nm wavelength, $100 \mathrm{~mW}$ \\
\hline Blue laser & DHOM-M-473-150 & 473nm wavelength, $160-195 \mathrm{~mW}$ \\
\hline Cyan dichroic filter & Permacolor \#5590 cyan & $\begin{array}{l}\text { Trans. AOI 0 : } 660 \mathrm{~nm}-0 \%, 540 \mathrm{~nm}- \\
81 \%, 480 \mathrm{~nm}-95 \%\end{array}$ \\
\hline $\begin{array}{l}\text { Light yellow green dichroic } \\
\text { filter }\end{array}$ & Permacolor \#4959 It. yellow green & $\begin{array}{l}\text { Trans. AOI 0 : } 660 \mathrm{~nm}-2 \%, 540 \mathrm{~nm}- \\
99 \%, 480 \mathrm{~nm}-23 \%\end{array}$ \\
\hline Plano-convex lens & Edmund Optics & Focal length $166 \mathrm{~mm}, \Phi=79 \mathrm{~mm}$ \\
\hline Plano-concave lens & Edmund Optics & Focal length $33 \mathrm{~mm}, \Phi=31 \mathrm{~mm}$ \\
\hline \multicolumn{3}{|l|}{ Overhanging beam } \\
\hline Metallic beam & $3 / 32 " \times 1 "$ - length $2 \mathrm{ft}$ & 3/32" Thick ( $\pm 0.001 ")$ \\
\hline Micrometer & Fowler 54-901-202 Digitrix 1-2" & $0.0001 "$ resolution \\
\hline \multicolumn{3}{|c|}{ Two-axis shearography interferometer } \\
\hline Piezo element & Stack piezo actuator $3 \times 3 \times 5 \mathrm{~mm}$ & $4 \mu \mathrm{m}$ at $150 \mathrm{~V} \pm 10 \%$ \\
\hline Mirror & first surface mirror & $51 \times 51 \times 6 \mathrm{~mm}$ \\
\hline Optical bracket & Newport U100-P2K-NL & Range $\pm 7^{\circ}$, sensitivity $3-6$ arc sec \\
\hline Beamsplitter cube & Anchor Optics AX27998 & $50 / 50$, size $45 \mathrm{~mm}$ \\
\hline Cyan dichroic filter & $\begin{array}{l}102272855 \text { Cyan 570nm (SWP) } \\
50 \times 50 \times 0.7 \mathrm{~mm}\end{array}$ & $\begin{array}{l}\text { Trans. AOI } 0^{\circ}: 660 \mathrm{~nm}-0 \%, 532 \mathrm{~nm}- \\
98.1 \%, 473 \mathrm{~nm}-98.2 \%\end{array}$ \\
\hline \multicolumn{3}{|l|}{ Camera system } \\
\hline Color camera & Prosilica GE680C & $640 \times 480$, cell size $7.4 \mu \mathrm{m}$ \\
\hline Macro lens & Navitar zoom 7000 & Focal length $18-108 \mathrm{~mm}$ \\
\hline
\end{tabular}

The RGB laser source idea comes from the need of having three lasers originating from the same place in space. Dealing with only one common source coordinate removes the complications arising from the 
use of multiple angles of illumination. The RGB laser is more convenient to manipulate and to position. The three colored lights need to be collimated, such the rays of light are parallel, to avoid having a varying sensitivity vector. A non-collimated source of light reaches the object's surface at different angles. This phenomenon could be mathematically corrected with the risk of increasing errors in the shearographic measurement knowing that the relative phase change depends on the angle of illumination. As shown in Figure 31, each laser has its own diverging lens, mainly for safety reasons to avoid having a concentrated beam leaving the laser head. Two dichroic filters are used at $45^{\circ}$ to co-align the three laser beams. A plano-concave lens is then used to enlarge the RGB beam. Finally, the beam is collimated with a plano-convex lens. A piece of black cardboard with a $50 \mathrm{~mm}$ diameter hole trimmed the edge of the Gaussian profile of the beam just before collimation to retain the relatively uniform central part remaining from the beam.
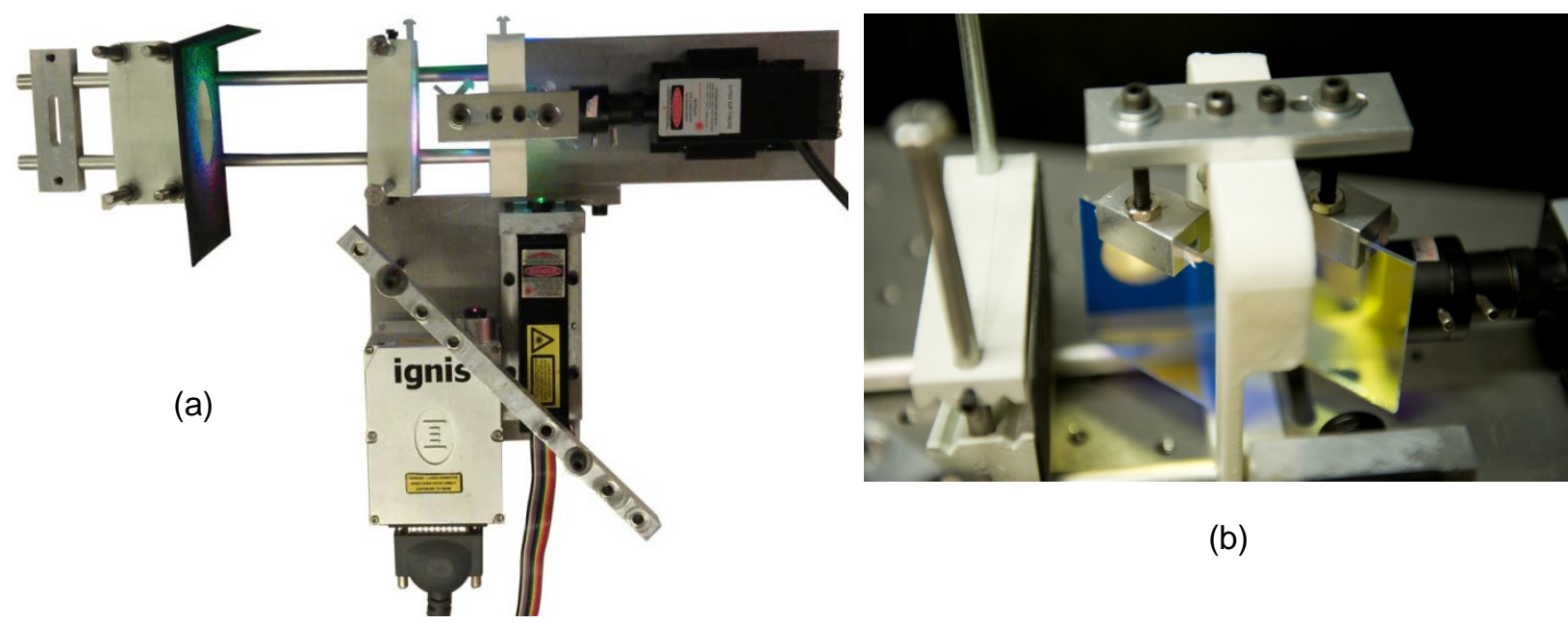

(b)

Figure 31 - RGB laser source (a) global view (b) dichroic filters for beam combination.

The overhanging beam test system illustrated in Figure 32 was specially designed to create very small displacements of the beam in the region of interest using significantly larger motions at the other end of the beam controlled by the micrometer. This arrangement allowed the micrometer to produce accurate and repeatable micro-scale beam motions. The beam specimen rests on two flat bars. When pushing at the extreme left side of the beam with the micrometer, the left side of the beam starting from the central support deflects away from the interferometer. From the right side of the central support, the beam deflects toward the interferometer. This area is the region of interest. The beam surface was painted with a thin layer of white paint to improve surface reflectivity, thereby improving optical response and data quality. The displacement-controlled design is a significant feature of the overhanging beam system because this arrangement enables accurately known displacements to be created without accurately knowing the Young's modulus and second moment of area. All that is required is for the beam to be made 
of a uniform material and to have a constant width and thickness along its entire length. A precisionground rectangular beam with tight tolerances was chosen to achieve this requirement.

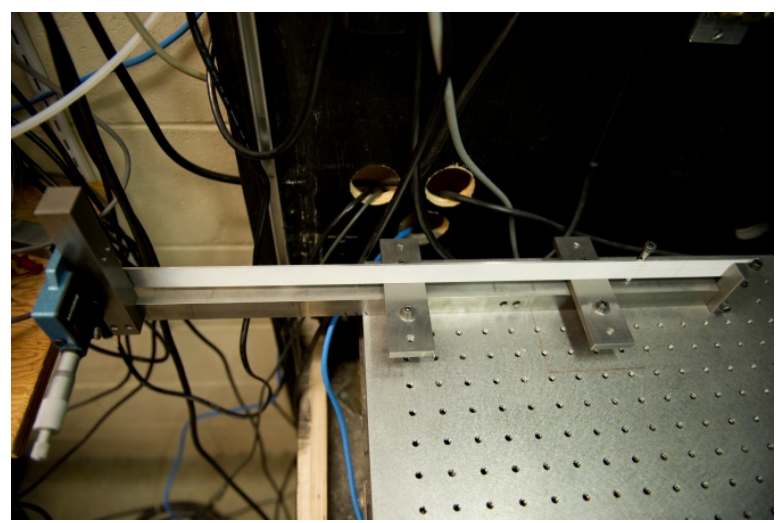

(a)

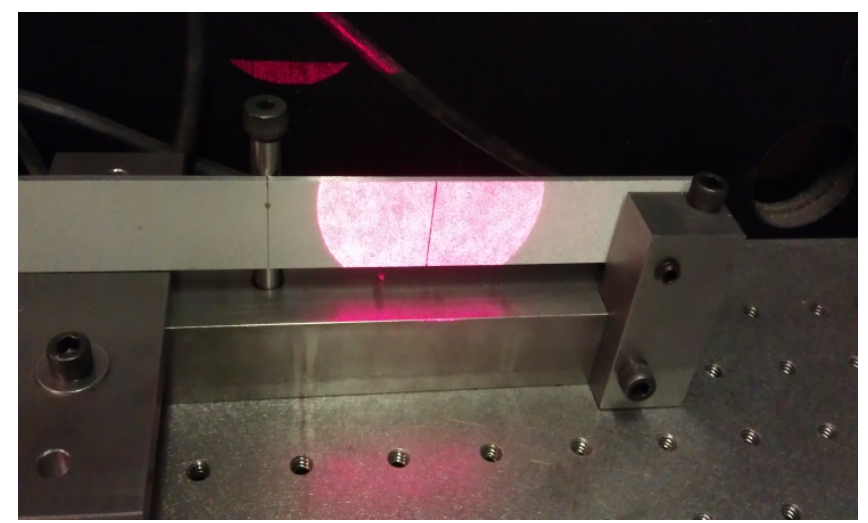

(b)

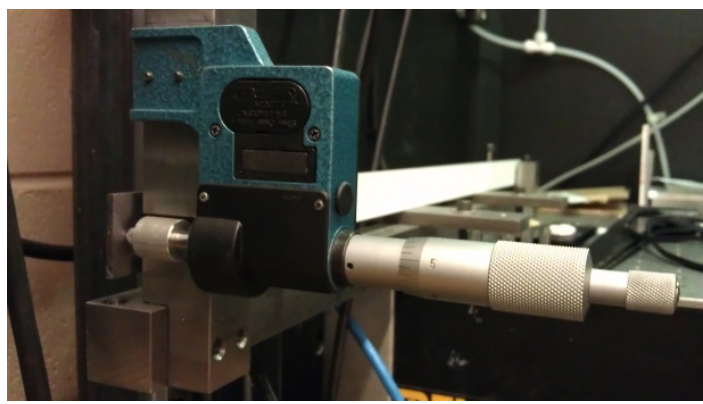

(c)

Figure 32 - Overhanging beam test system (a) global view (b) region of interest with red light only (c) modified micrometer.

The two-axis shearography interferometer includes two modified Michelson interferometers in one. As displayed in Figure 33, the first interferometer encompasses two optical brackets, three piezo stacks, two thick mirrors, and a beamsplitter cube, which let $50 \%$ of the incoming light going through while the rest is reflected. A cyan dichroic filter between one of the mirrors and the beamsplitter cube is added to the system to get a second fully functional interferometer. The $0.7 \mathrm{~mm}$ thick dichroic filter is directly fixed to the beamsplitter cube. The arm lengths of both interferometers need to be as similar as possible so that both pairs of shifted images remain within the short depth of focus of the macro lens. On Figure 33(a), both mirrors are mounted on piezo element but only one mirror is stepped during the measurement. Three-point optical brackets with fine screws allow the shear distance to be easily adjusted. 


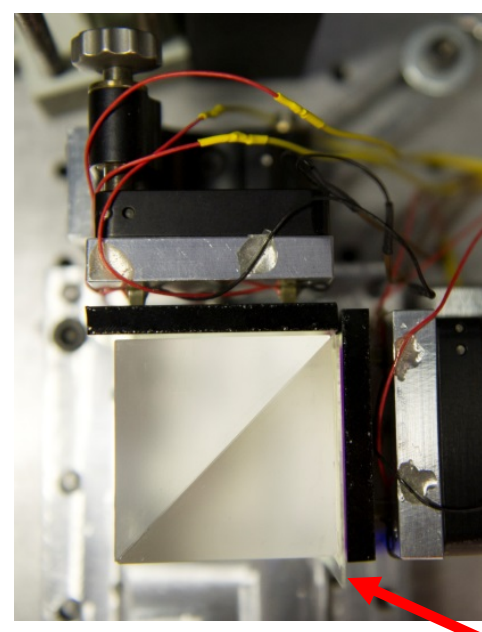

(a)

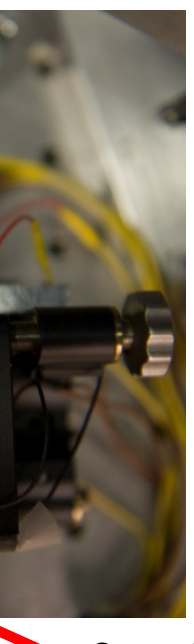

Cyan dichroic filter

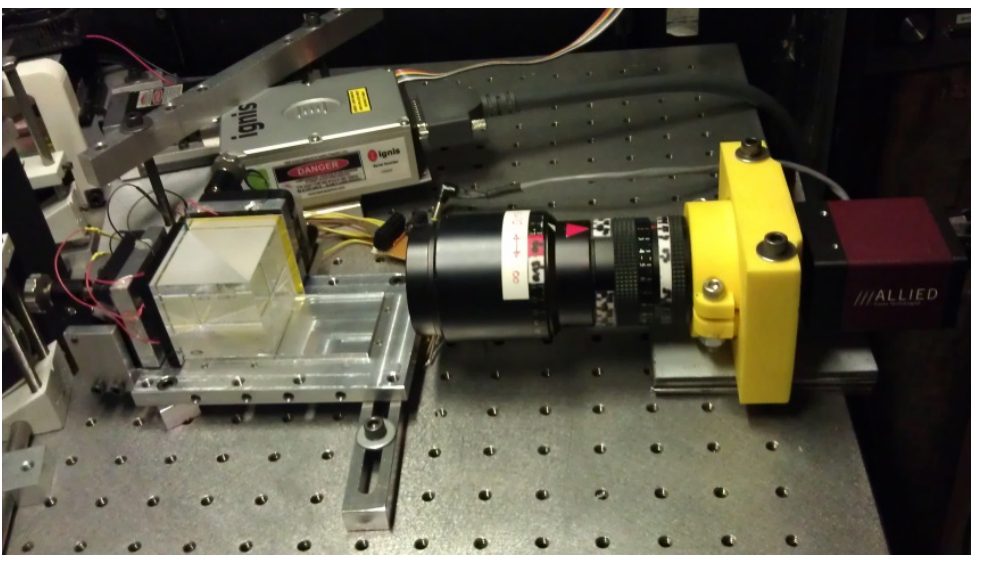

(b)

Figure 33 - Two-axis shearography interferometer (a) interferometer close up, (b) interferometer with imaging system.

\subsection{Overhanging Beam Measurements}

\subsubsection{Beam Theoretical Deformation}
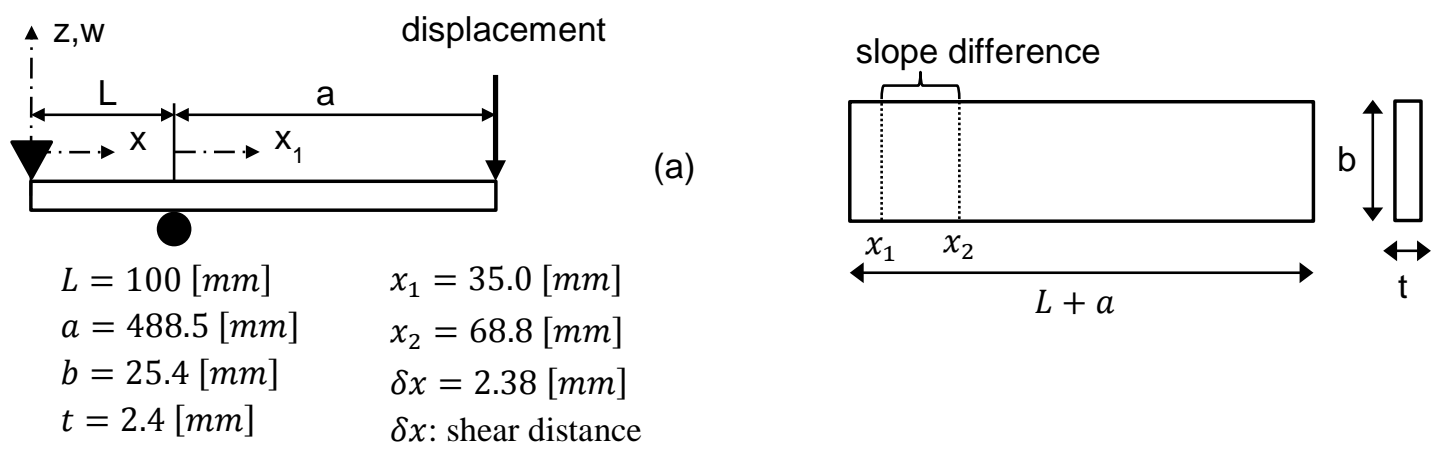

(b)

Figure 34 - Overhanging beam (a) plan view (b) side view.

To demonstrate the concept of making simultaneous ESPI measurements using multiple wavelengths and a color camera an overhanging beam apparatus was designed and built as shown in Figure 32. The tight-tolerance beam has dimensions $610 \times 25.4 \times 2.38 \mathrm{~mm}$. According to the Euler beam theory [100], the beam out-of-plane displacement is:

$$
w=\frac{F a x}{6 E I L}\left(L^{2}-x^{2}\right) \text { for }(0 \leq x \leq L)
$$




$$
\begin{aligned}
& w_{1}=\frac{F x_{1}}{6 E I}\left(2 a L+3 a x_{1}-x_{1}{ }^{2}\right) \text { for }\left(0 \leq x_{1} \leq a\right) \\
& w_{1}=\frac{F(L+a)}{6 E I}\left[2 a L+3 a x_{1}-(L+a)^{2}\right] \text { for }(L \leq x \leq(L+a))
\end{aligned}
$$

The force can be expressed as:

$$
F=\frac{w_{1} 6 E I}{2 a^{2} L+2 a^{3}}
$$

with $d w_{1}$ being the imposed displacement at the location $x=L+a$. The out-of-plane displacement for the region of interest located within the distance $L$ from Figure $34(\mathrm{a})$ can be rewritten:

$$
w=\frac{w_{1} x\left(L^{2}-x^{2}\right)}{\left(2 a L+2 a^{2}\right) L}
$$

The first derivative of the out-of-plane deformation is:

$$
\begin{aligned}
& \frac{d w}{d x}=\frac{F a}{6 E I L}\left(L^{2}-3 x^{2}\right) \text { for }(0 \leq x \leq L) \\
& \frac{d w}{d x}=\frac{w_{1} a\left(L^{2}-3 x^{2}\right)}{\left(2 a^{2} L+2 a^{3}\right) L} \quad \text { for } \quad(0 \leq x \leq L)
\end{aligned}
$$

As shown in Figure 35, the region of interest is located between $x_{1}$ and $x_{2}$, which corresponds to the right and left edges of the captured images. The cone-point set screw is positioned at $0 \mathrm{~mm}$ on the overhanging beam while the shoulder screw is placed at $100 \mathrm{~mm}$, thus the region of interest sits in the middle of the portion between both screws to avoid stress concentrations. The portion of the beam between the shoulder screw and the micrometer is relatively long to ensure a high sensitivity on the beam deformation.

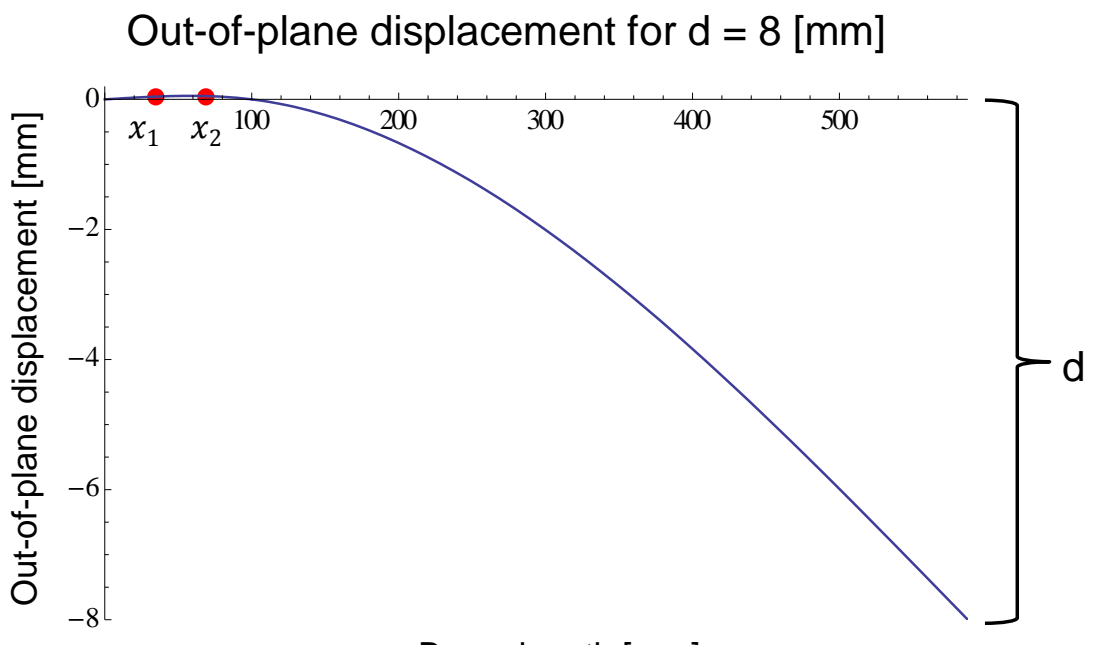

Beam length [mm]

Figure 35 - Overhanging beam out-of-plane displacement for an $8 \mathrm{~mm}$ displacement on the micrometer. 


\subsubsection{Slope Measurements}

An initial test of the color separation method of Equation (4) was made. The three lasers red $\lambda=660 \mathrm{~nm}$, green $\lambda=532 \mathrm{~nm}$, and blue $\lambda=473 \mathrm{~nm}$ were sequentially used to illuminate a white test specimen. Measurements were made using a Prosilica GE680C color camera (Allied Vision Techologies, Inc, Burnaby, Canada). The raw individual color images from the camera showed cross-talk between color measurements in the 5-15\% range. After application of Equation (4), the cross-talk was reduced to around $1 \%$. Thus, the matrix inversion method for calibrating the color separation was found to be effective.

After completion of the color calibration, a test was done to assess the ability of the color camera to do simultaneous ESPI measurements. This was done by using a single-axis shearography interferometer as shown Figure 19 to measure the surface slope of the overhanging beam shown in Figure 32. Two lasers, red and blue simultaneously illuminated the same beam area between $x_{1}$ and $x_{2}$ in Figure 34(b). The micrometer screw applied a sequence of $8 \times 1 \mathrm{~mm}$ displacement increments at the right side of the beam in Figure 34(a). Evaluation of the slope difference between two points $x_{1}$ and $x_{2}$ avoids the need to determine the absolute slope.

The speckle size needs approximately to match the pixel size of the camera sensor to ensure maximum intensity variations on the pixels. Based on the Airy disk diameter, the approximate size of a speckle is $\sim 1.2 \mathrm{~F} / \lambda$ [101]. The pixel size of the Prosilica GE680C camera is $7.4 \mu \mathrm{m}$, so for red laser light with $\lambda=$ $0.660 \mu \mathrm{m}$ the required F-number is 4.1 . A larger F-number $=8$ was chosen for the experiments to provide some oversampling. The exposure time is set around 350ms to get an adequate camera response. Some care had to be taken to adjust the light intensity of each laser so that the recorded speckle patterns have approximately similar average intensity.

Figure 36(a) shows the wrapped phase map extracted from the blue image; it contains 15.4 vertical fringes. Figure $36(\mathrm{~b})$ shows the corresponding wrapped phase map extracted from the red image; it contains 11.6 fringes. The two phase maps measure the same deformation and therefore the fringe numbers should be in the inverse ratio of the wavelengths. Here, $N_{\text {blue }} / N_{\text {red }}=1.33$ and $\lambda_{\text {red }} / \lambda_{\text {blue }}=1.39$. In Figure 36(c), the Mean Absolute Percentage Error (MAPE) is around 2\% for the red and blue signal. The max Absolute Percentage Error (APE) is below $5 \%$ for both colors. 


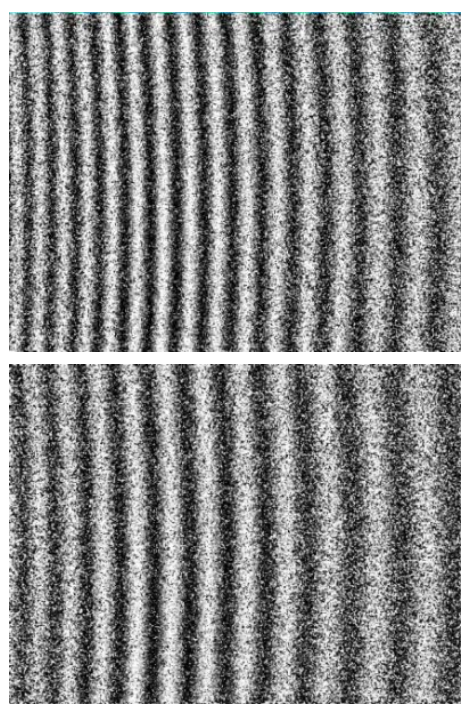

(a)

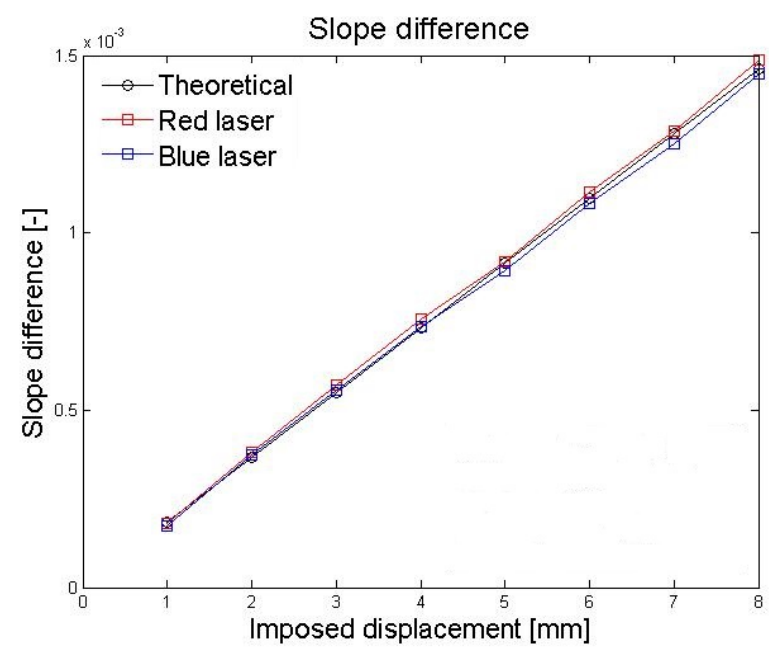

(c)

(b)

Figure 36 - Slope difference measurements in $x$ direction using a single-axis interferometer (a) wrapped phase map blue laser, (b) wrapped phase map red laser, (c) slope difference results.

A further measurement was done to illustrate two-direction shearography using the arrangement shown in Figure 22. The same overhanging beam was used as in single-axis interferometer measurement. In this two-direction case, the measured fringes differ not only in their spacing but also in their orientation. The region of interest is located between $x_{1}=41.4 \mathrm{~mm}$ and $x_{2}=72.3 \mathrm{~mm}$ with a shear distance in the $\mathrm{x}$ direction of $2.984 \mathrm{~mm}$ corresponding to $9.7 \%$ of the image's width and with a shear distance in the $y$ direction of $1.960 \mathrm{~mm}$ corresponding to $8.5 \%$ of the image's height. The fringes in Figure $37(\mathrm{a})$ indicate the surface slope in the longitudinal direction of the beam, with approximately 15.8 vertical fringes for the blue image. Figure $37(\mathrm{~b})$ shows the corresponding wrapped phase map extracted from the green image; it comprises 14.1 fringes. The two phase maps measure the same deformation and therefore the fringe numbers should be in the inverse ratio of the wavelengths. Here, $N_{\text {blue }} / N_{\text {green }}=1.12$ and $\lambda_{\text {green }} / \lambda_{\text {blue }}=$ 1.12. The fringes in Figure 37(c) indicate the surface slope in the transverse direction of the beam, with approximately 2 horizontal fringes. These fringes represent the surface anticlastic curvature of the beam, a phenomenon controlled by the action of Poisson's ratio [102]. The red laser light is reflected from the cyan dichroic filter which acts like a mirror for the unwanted spectrum but this filter is not perfectly flat creating thus a non-uniform shear for the red signal. The red interferogram is mathematically corrected including filtering to avoid unwrapping errors. This explains why the horizontal fringes are presented being filtered. In Figure 37(d), the mean absolute percentage error is around 1.4\% for the blue results over the eight measurements. The max absolute percentage error remains below $3.4 \%$ for the blue results. In Figure $37(\mathrm{e})$, the mean absolute percentage error is around $1.4 \%$ for the green results over the eight measurements. The max absolute percentage error remains below $5.9 \%$ for the green results. 


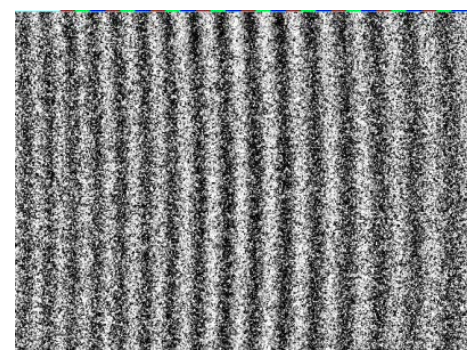

(a)

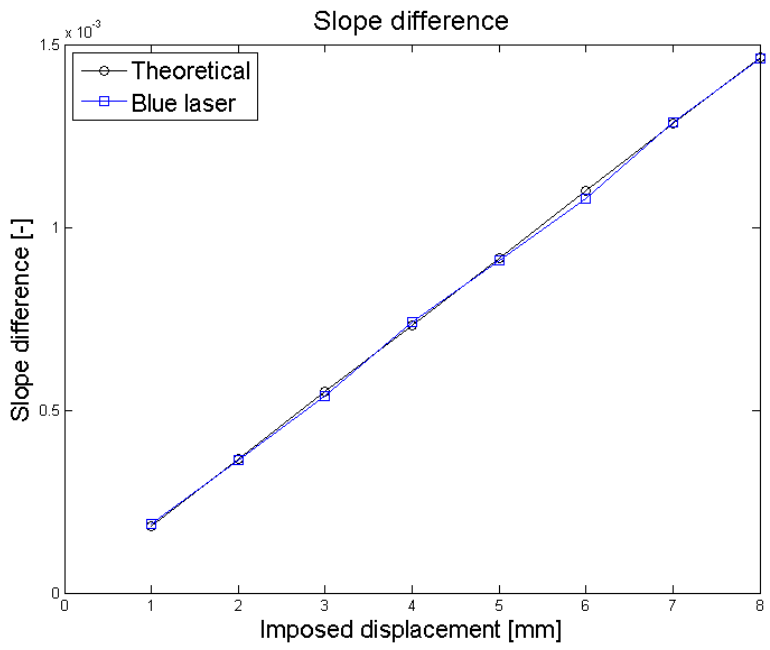

(d)

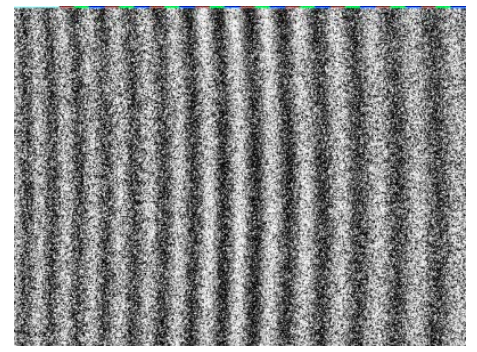

(b)
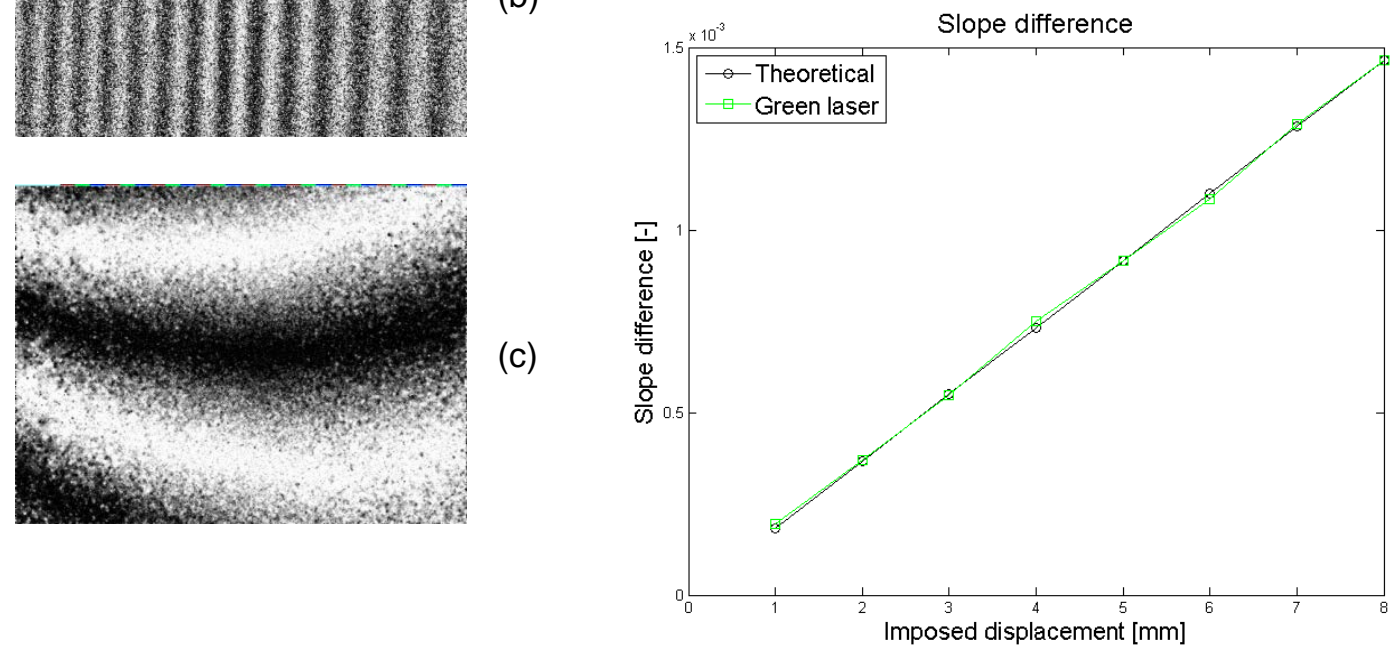

(e)

Figure 37 - Slope difference measurements with a two-axis interferometer (a) wrapped phase map blue laser $=\partial w / \partial x,(b)$ wrapped phase map green laser $=\partial w / \partial x,(c)$ wrapped phase map red laser $=\partial w / \partial y$ with filtering, (d) slope difference results for blue laser, (e) slope difference results for green laser.

This second slope measurement does not only contain the slope difference but also the relative surface slope over the entire region of interest comprised between $x_{1}=41.4 \mathrm{~mm}$ and $x_{2}=72.3 \mathrm{~mm}$. Since the measured phase is mainly varying according the $\mathrm{x}$-direction or along the beam length, the relative slope matrices have been averaged column by column and then compared with theoretical values. Figure 38 shows the relative slope measured with blue and green signals across the entire region of interest for an $8 \mathrm{~mm}$ imposed displacement on the beam. The Mean Absolute Error (MEA) is around 8.3 microstrain while the max Absolute Error ( $\mathrm{AE}$ ) is below 48 microstrain for the blue results. Concerning the green results, the mean absolute error is around 7.0 microstrain and the max absolute error remains below 21 microstrain. 


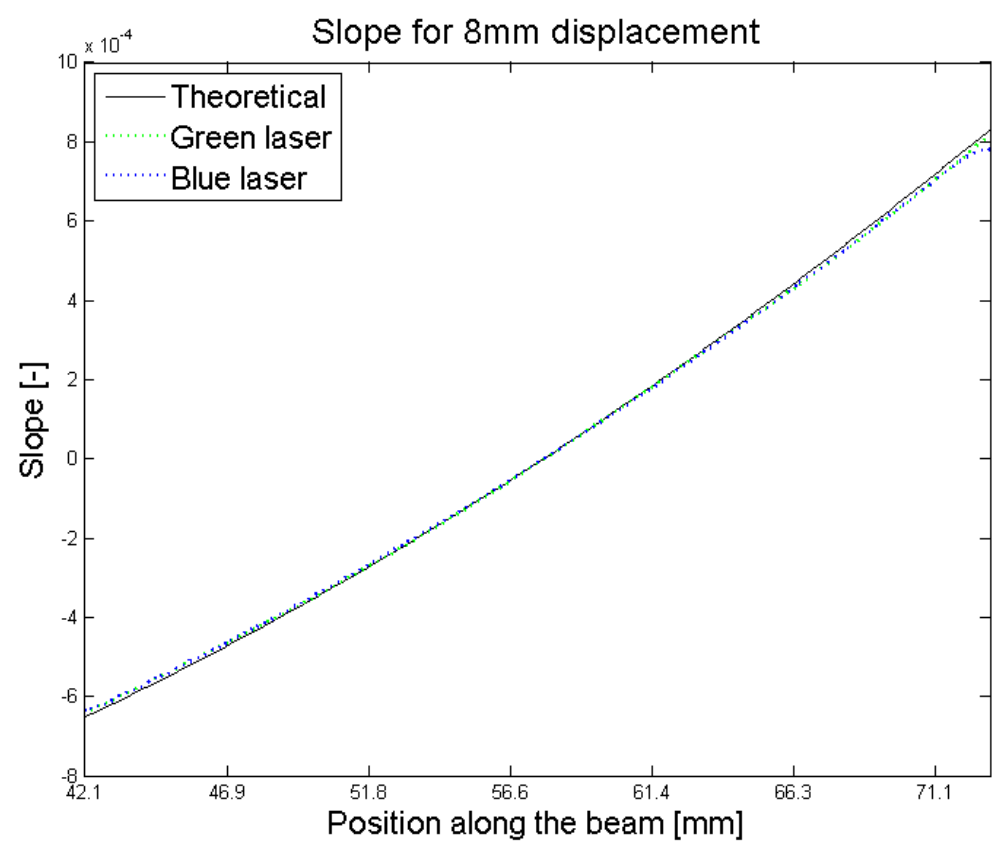

Figure 38 - Relative slope of blue and green laser for an 8mm imposed displacement.

Table 3 summarizes the relative surface slope errors for a sequence of $8 \times 1 \mathrm{~mm}$ displacement imposed on the beam. The mean absolute error corresponds to the average error over the entire image. The max absolute error is the maximum error over the slope averaged column by column and it reflects the noise that appears across the image, particularly on the edges. For example, the maximum absolute error, for the blue color shown in Figure 38 is relatively high because the right side of the blue interferogram contains substantial noise.

Table 3 - Absolute errors on the relative slope measured with green and blue.

\begin{tabular}{|c|c|c|c|c|c|c|c|c|c|}
\cline { 2 - 9 } \multicolumn{2}{c|}{} & $\begin{array}{c}1 \mathrm{~mm} \\
{[\mu \text { strain }]}\end{array}$ & $\begin{array}{c}2 \mathrm{~mm} \\
{[\mu \text { strain] }}\end{array}$ & $\begin{array}{c}3 \mathrm{~mm} \\
{[\mu \text { strain }]}\end{array}$ & $\begin{array}{c}4 \mathrm{~mm} \\
{[\mu \text { strain }]}\end{array}$ & $\begin{array}{c}5 \mathrm{~mm} \\
{[\mu \text { strain }]}\end{array}$ & $\begin{array}{c}6 \mathrm{~mm} \\
{[\mu \mathrm{strain}]}\end{array}$ & $\begin{array}{c}7 \mathrm{~mm} \\
{[\mu \text { strain }]}\end{array}$ & $\begin{array}{c}8 \mathrm{~mm} \\
{[\mu \text { strain }]}\end{array}$ \\
\hline \multirow{2}{*}{ blue } & MAE & 0.8 & 3.3 & 5.8 & 1.9 & 6.3 & 10.2 & 5.1 & 8.3 \\
\cline { 2 - 10 } & Max AE & 12.4 & 12.2 & 12.7 & 9.8 & 21.2 & 33.9 & 33.4 & 47.4 \\
\hline \multirow{2}{*}{ green } & MAE & 1.6 & 1.4 & 3.3 & 0.9 & 4.2 & 8.6 & 4.0 & 7.0 \\
\cline { 2 - 10 } & Max AE & 4.0 & 8.1 & 15.5 & 5.7 & 12.1 & 23.8 & 13.5 & 20.1 \\
\hline
\end{tabular}

\subsubsection{Absolute Phase Measurements}

The absolute phase measurement is done simultaneously as the second slope measurement case described in Section 5.2.2. The green and red signals are used to extract the surface slope while the blue laser light is used redundantly with the green light to measure the absolute phase with respect to the $x$ direction. Speckled images are recorded before and after loading and the two-wavelength method, described earlier, is applied using green and blue interferograms to determine the absolute phase. 
The phase variation being mainly relative to the $x$-direction, the measured phase matrices have been averaged column by column and then compared with theoretical values. The value $s$ from Equation (56) is calculated with the entire green and blue matrices to avoid noise. The results are presented in graphs where the $y$-axis represents the phase in $2 \pi$ cycles.

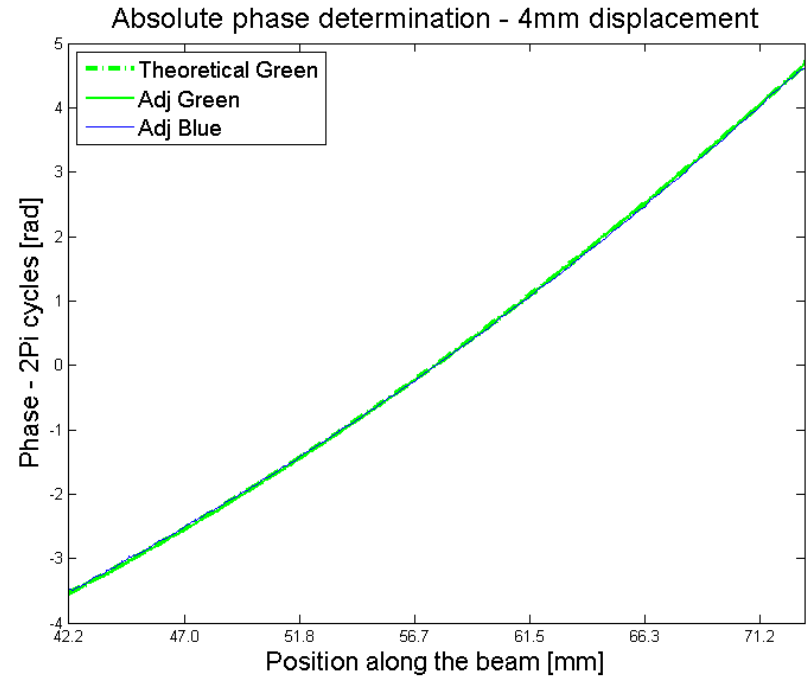

(a)

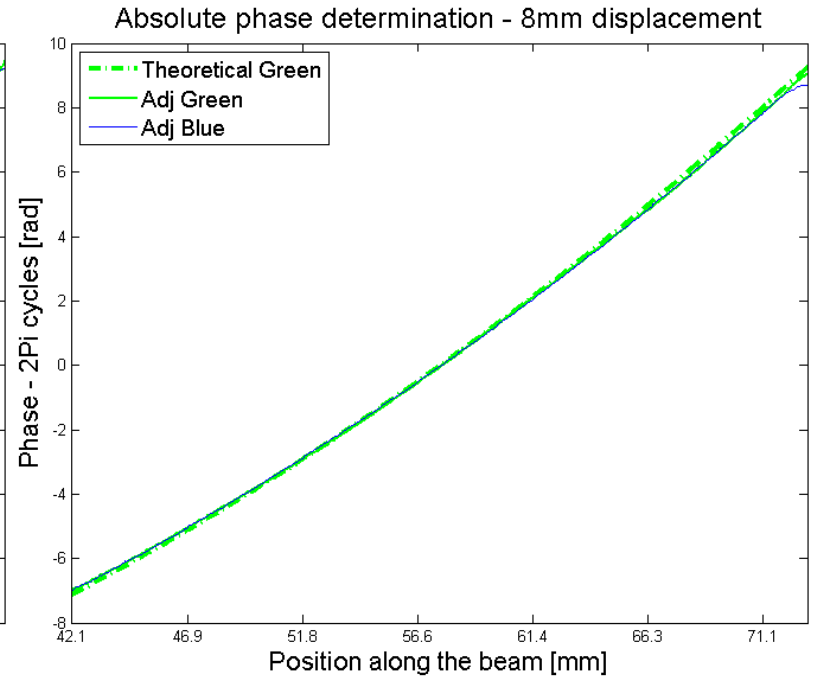

(b)

Figure 39 - Absolute phase determination for (a) 4mm and (b) $8 \mathrm{~mm}$ imposed displacement.

The theoretical green phase, plotted with a long-dash-dot line, can be retrieved with the measured blue and green signals. According to Equation (54), the absolute green phase could be calculated in two different ways, the first being:

$$
\text { Adj Green }=\varphi-2 \pi m
$$

where $\varphi$ is the measured green relative phase change. The second calculation possibility is:

$$
\text { Adj Blue }=(\tau-2 \pi n)(1-\alpha)=(\tau-2 \pi n) \frac{\lambda_{\text {blue }}}{\lambda_{\text {green }}}
$$

where $\tau$ is the measured blue relative phase change.

In Figure 39(a), the mean absolute error is around 0.02 cycle of $2 \pi$ for the blue results and 0.01 cycle of $2 \pi$ for the green results with $4 \mathrm{~mm}$ imposed displacement. The max absolute error is around 0.11 cycle of $2 \pi$ for the blue results and 0.06 cycle of $2 \pi$ for the green results with $4 \mathrm{~mm}$ imposed displacement. Figure 39 (b) shows the results with $8 \mathrm{~mm}$ imposed displacement where the MAE is 0.09 cycle of $2 \pi$ for the blue and 0.07 cycle of $2 \pi$ for the green. The max AE reaches 0.52 cycle of $2 \pi$ for the blue and 0.22 cycle of $2 \pi$ for the green color. If a jump of $2 \pi$ is considered as a $100 \%$ error, the mean absolute percentage error remains under $12 \%$ for both colors over the eight measurements, summarized in Table 4. 
Table 4 - Absolute errors on the absolute green phase measured with green and blue.

\begin{tabular}{|c|c|c|c|c|c|c|c|c|c|}
\cline { 2 - 10 } \multicolumn{2}{c|}{} & $\begin{array}{c}1 \mathrm{~mm} \\
{[2 \pi \text { cycles }]}\end{array}$ & $\begin{array}{c}2 \mathrm{~mm} \\
{[2 \pi \text { cycles }]}\end{array}$ & $\begin{array}{c}3 \mathrm{~mm} \\
{[2 \pi \text { cycles }]}\end{array}$ & $\begin{array}{c}4 \mathrm{~mm} \\
{[2 \pi \text { cycles }]}\end{array}$ & $\begin{array}{c}5 \mathrm{~mm} \\
{[2 \pi \text { cycles }]}\end{array}$ & $\begin{array}{c}6 \mathrm{~mm} \\
{[2 \pi \text { cycles }]}\end{array}$ & $\begin{array}{c}7 \mathrm{~mm} \\
{[2 \pi \text { cycles }]}\end{array}$ & $\begin{array}{c}8 \mathrm{~mm} \\
{[2 \pi \text { cycles }]}\end{array}$ \\
\hline \multirow{2}{*}{ blue } & MAE & 0.01 & 0.04 & 0.06 & 0.02 & 0.07 & 0.11 & 0.06 & 0.09 \\
\cline { 2 - 11 } & Max AE & 0.13 & 0.14 & 0.14 & 0.11 & 0.23 & 0.37 & 0.37 & 0.52 \\
\hline \multirow{2}{*}{ green } & MAE & 0.02 & 0.02 & 0.04 & 0.01 & 0.05 & 0.09 & 0.04 & 0.07 \\
\cline { 2 - 11 } & Max AE & 0.04 & 0.09 & 0.17 & 0.06 & 0.13 & 0.26 & 0.15 & 0.22 \\
\hline
\end{tabular}

\subsubsection{Deformed Shape Measurements}

The deformed shape measurement is also performed at the same time as the second slope measurement case described in Section 5.2.2. It is possible to measure the actual surface shape of the specimen being under investigation by moving the illumination source, where images are recorded before and after moving the source of light. Here, the main goal is to prove the concept of using multiple wavelengths and a color camera for doing ESPI measurement. Shearography was chosen for its convenience and great applicability in the industry. This research tries to extract as many information in a single measurement as possible. So far, the relative slope measurement and the absolute phase measurement, which leads to the absolute slope, have been demonstrated. Working with multiple colors also permits to acquire the deformed shape of the test object. One would find to get the original shape of the specimen the most adequate for his application but this is not the goal here.

The shape reconstruction is using red and green wavelengths. The blue signal does not contribute to that calculation in the present work mainly because the red and green lasers were of a higher quality and were more stable and less noisy than the blue laser. As displayed in Figure 40(a-b-c), the mean absolute error for a $4 \mathrm{~mm}$ imposed displacement on the beam is $0.25 \mu \mathrm{m}$ and the max absolute error is $0.57 \mu \mathrm{m}$. For an $8 \mathrm{~mm}$ imposed displacement, the mean absolute error almost double to reach $0.49 \mu \mathrm{m}$ and the max absolute error jumps to $1.24 \mu \mathrm{m}$. 


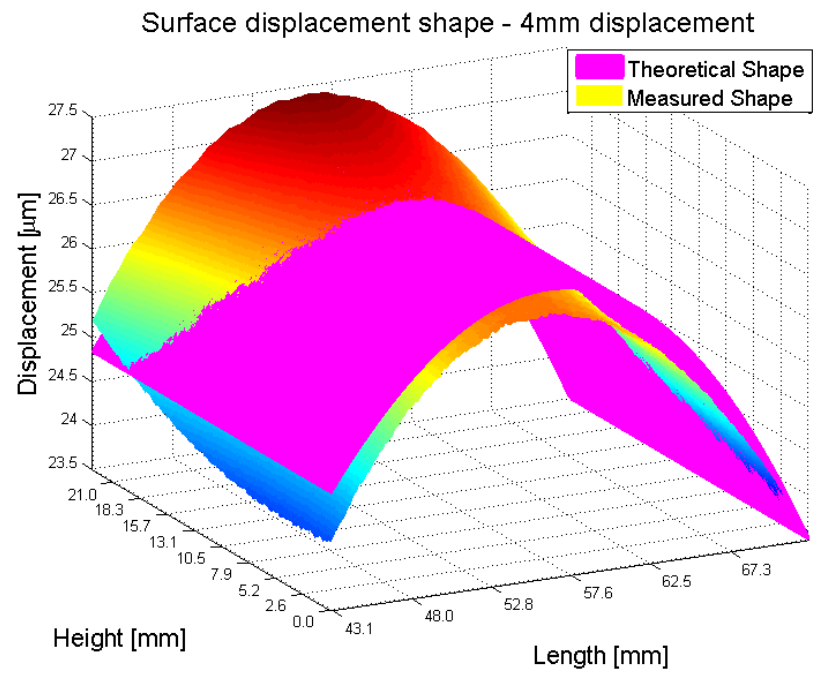

(a)

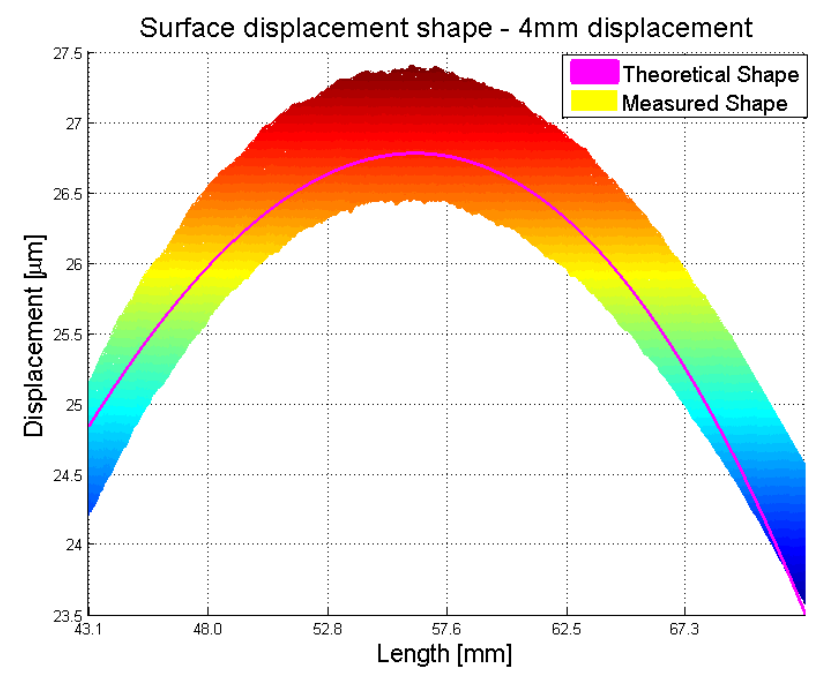

(b)

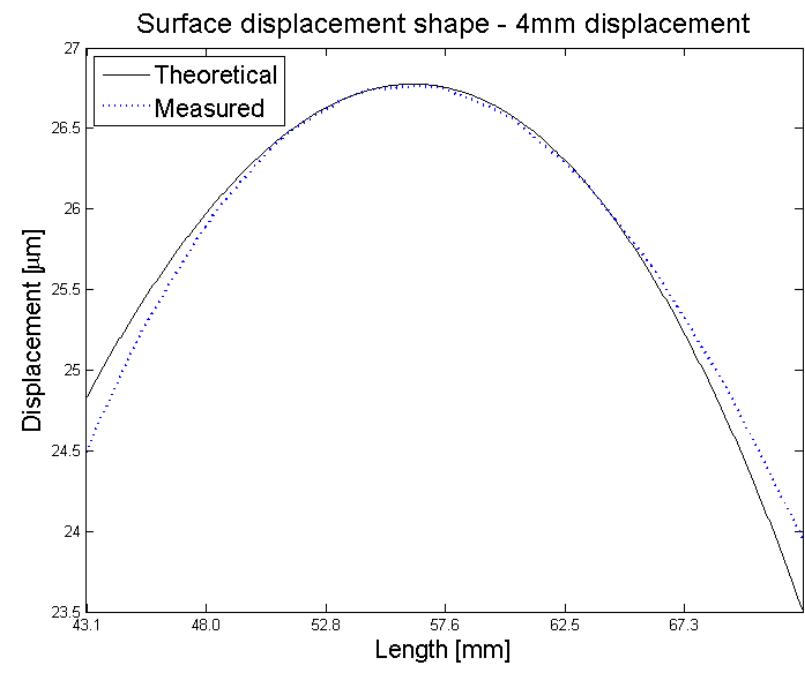

(c)

Figure 40 - Reconstructed surface displacement shape (a) orthogonal view for a $4 \mathrm{~mm}$ imposed displacement, (b) bottom view for $4 \mathrm{~mm}$, (c) longitudinal shape averaged across the width.

As found in Table 5, the mean absolute error increases as the imposed displacement on the beam rises. Considering only the mean absolute error, the shearographic system developed here is capable of measuring surface displacements with an accuracy in the range $0.1 \lambda$ and $\lambda$ with respect to the imposed displacement and according to theoretical values.

Table 5 - Absolute errors on the measured surface displacement shape with green and red.

\begin{tabular}{|c|c|c|c|c|c|c|c|c|c|}
\cline { 2 - 9 } \multicolumn{2}{c|}{} & $\begin{array}{c}1 \mathrm{~mm} \\
{[\mu \mathrm{m}]}\end{array}$ & $\begin{array}{c}2 \mathrm{~mm} \\
{[\mu \mathrm{m}]}\end{array}$ & $\begin{array}{c}3 \mathrm{~mm} \\
{[\mu \mathrm{m}]}\end{array}$ & $\begin{array}{c}4 \mathrm{~mm} \\
{[\mu \mathrm{m}]}\end{array}$ & $\begin{array}{c}5 \mathrm{~mm} \\
{[\mu \mathrm{m}]}\end{array}$ & $\begin{array}{c}6 \mathrm{~mm} \\
{[\mu \mathrm{m}]}\end{array}$ & $\begin{array}{c}7 \mathrm{~mm} \\
{[\mu \mathrm{m}]}\end{array}$ & $\begin{array}{c}8 \mathrm{~mm} \\
{[\mu \mathrm{m}]}\end{array}$ \\
\hline \multirow{2}{*}{ shape } & MAE & 0.07 & 0.13 & 0.20 & 0.25 & 0.31 & 0.34 & 0.42 & 0.49 \\
\cline { 2 - 11 } & Max AE & 0.18 & 0.27 & 0.45 & 0.57 & 0.77 & 0.93 & 1.12 & 1.24 \\
\hline
\end{tabular}




\subsubsection{Effect of the Correction for Non-Uniform Shear}

The previous results were all corrected for non-uniform shear with the algorithm described in Chapter 4.3.1 but they do not reveal the potential of that algorithm very well. To demonstrate the effectiveness of the non-uniform shear correction algorithm, a last measurement was performed where the tilt-mirror was oriented to get shear in $\mathrm{x}$ and $\mathrm{y}$-direction while the piezo-tilt mirror was only rotated to get images sheared only in $y$-direction. The blue and green signals are sheared in $x$-y-direction and the red signal is sheared in $y$-direction. This configuration simulates mostly for mirror misalignment. The region of interest is also located between $x_{1}=41.4 \mathrm{~mm}$ and $x_{2}=72.3 \mathrm{~mm}$ with a shear distance in the $\mathrm{x}$-direction of $2.981 \mathrm{~mm}$ and a shear distance in the $y$-direction of $1.995 \mathrm{~mm}$ for the blue-green signal. The red signal measuring the surface slope $\partial w / \partial y$ has a shear distance of $1.947 \mathrm{~mm}$ in the $y$-direction. The $1.995 \mathrm{~mm}$ unwanted $y$-shear corresponds to an $8.6 \%$ misalignment. The theoretical $0 \mathrm{~mm}$ shear distance for the red color in the $\mathrm{x}$ direction is in reality $0.019 \mathrm{~mm}$, which corresponds to a $0.08 \%$ misalignment. As shown in Figure 41 , the correction for non-uniform shear algorithm is seen to be successful in correcting the three different phase maps.

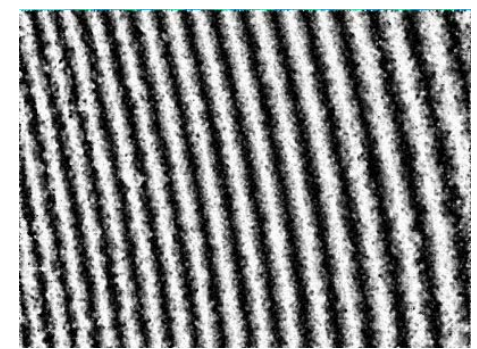

(a)

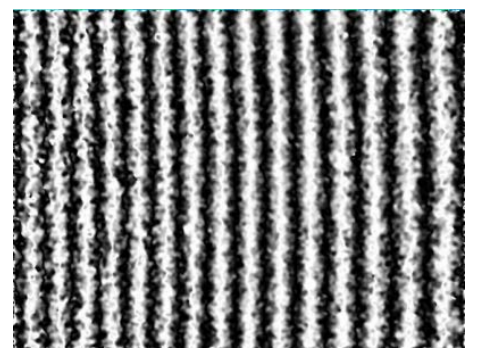

(b)
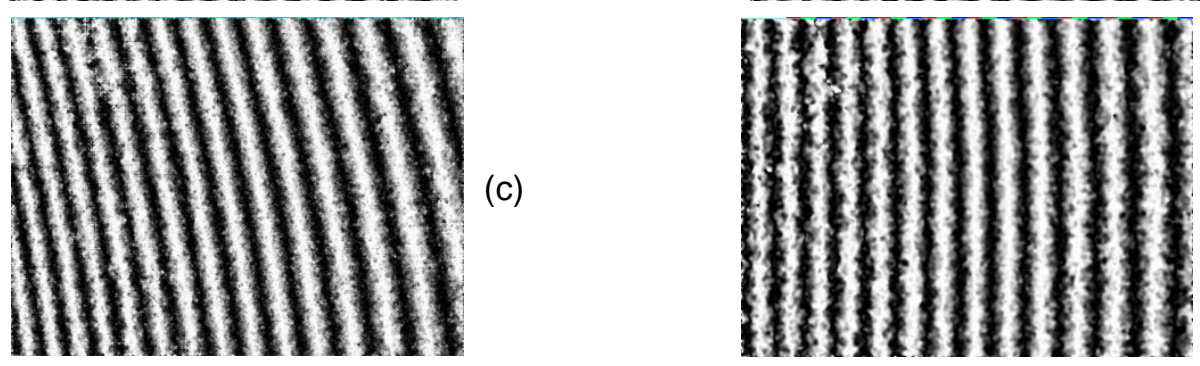

(d)
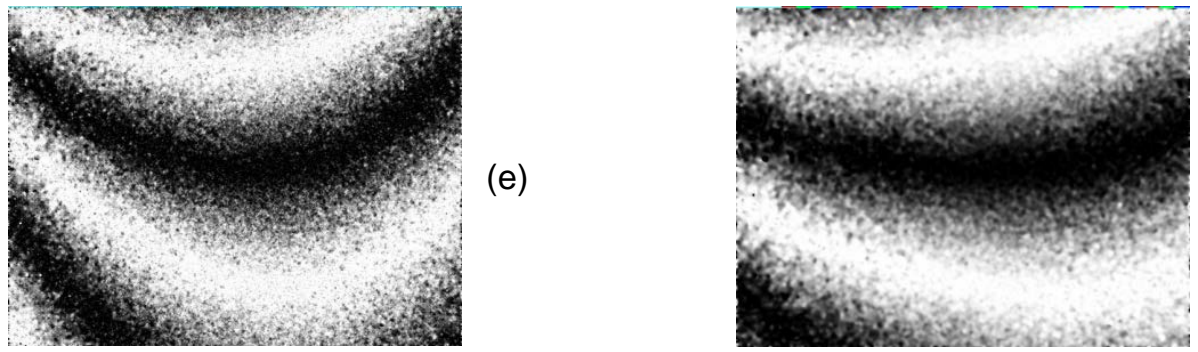

(e)

Figure 41 - Slope difference measurements with a two-axis interferometer (a) uncorrected wrapped phase map blue laser $=\partial w / \partial x$, (b) corrected wrapped phase map blue laser, (c) uncorrected wrapped phase map green laser $=\partial w / \partial x,(d)$ corrected wrapped phase map green laser, (e) uncorrected wrapped phase map red laser $=\partial w / \partial y,(f)$ corrected wrapped phase map red laser. 
In Figure 42(a), the measured slope difference for the blue color is presented where the mean absolute percentage error is around $3.2 \%$ over the eight measurements. The max absolute percentage error remains below $7.5 \%$ for the blue results. In Figure 42 (b), the mean absolute percentage error is around $2.4 \%$ for the green results over the eight measurements. The max absolute percentage error remains below $5.1 \%$ for the green results.

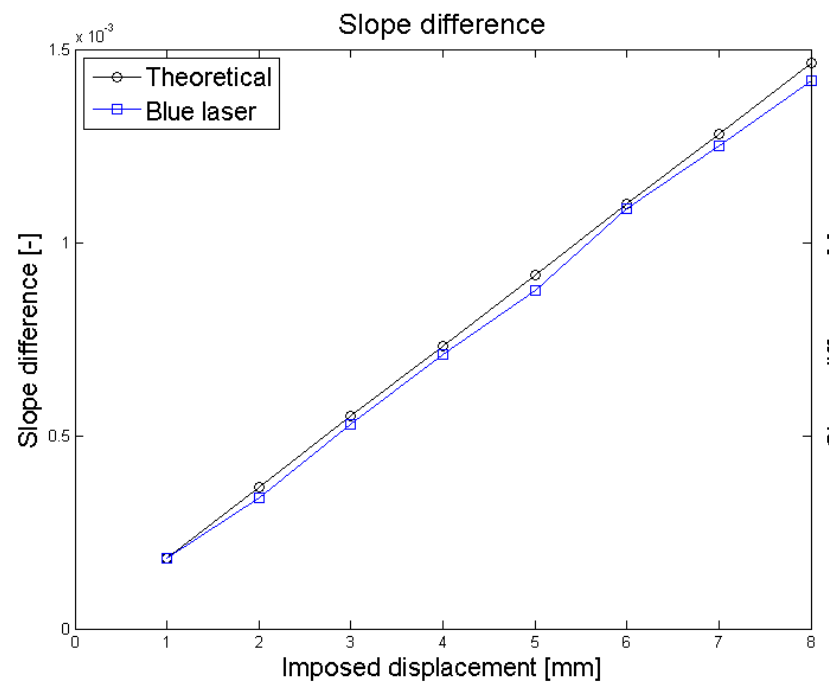

(a)

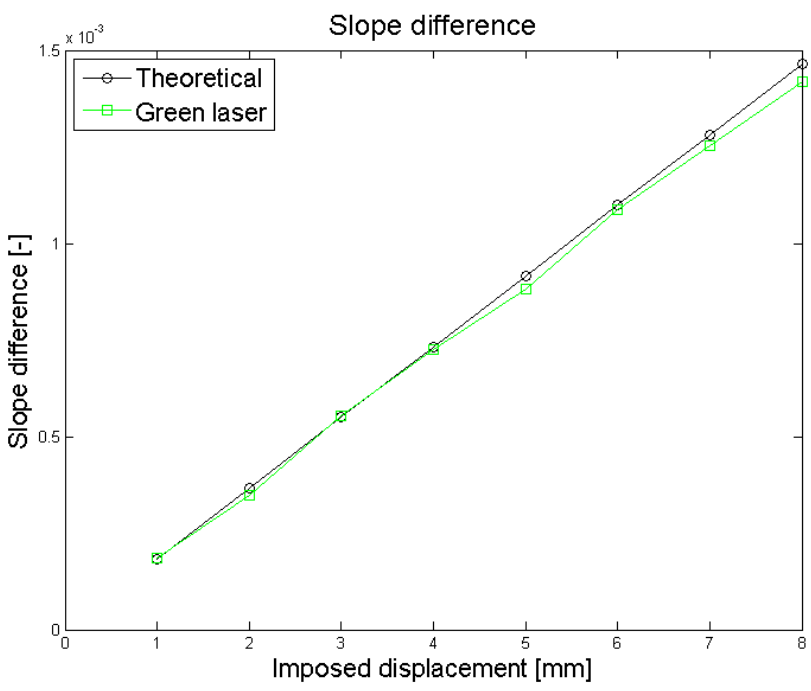

(b)

Figure 42 - Slope difference measurements with a two-axis interferometer (a) slope difference results for blue laser, (b) slope difference results for green laser.

Figure 43 shows the relative slope measured with blue and green signals across the entire region of interest for an $8 \mathrm{~mm}$ imposed displacement on the beam. The mean absolute error is around 22.1 microstrain, which is 2.7 times greater than without large misalignment, while the max absolute error is below 106 microstrain for the blue results. Concerning the green results, the mean absolute error is around 23.3 microstrain, which is 3.3 times greater than without large misalignment, and the $\max$ absolute error remains below 117 microstrain. 


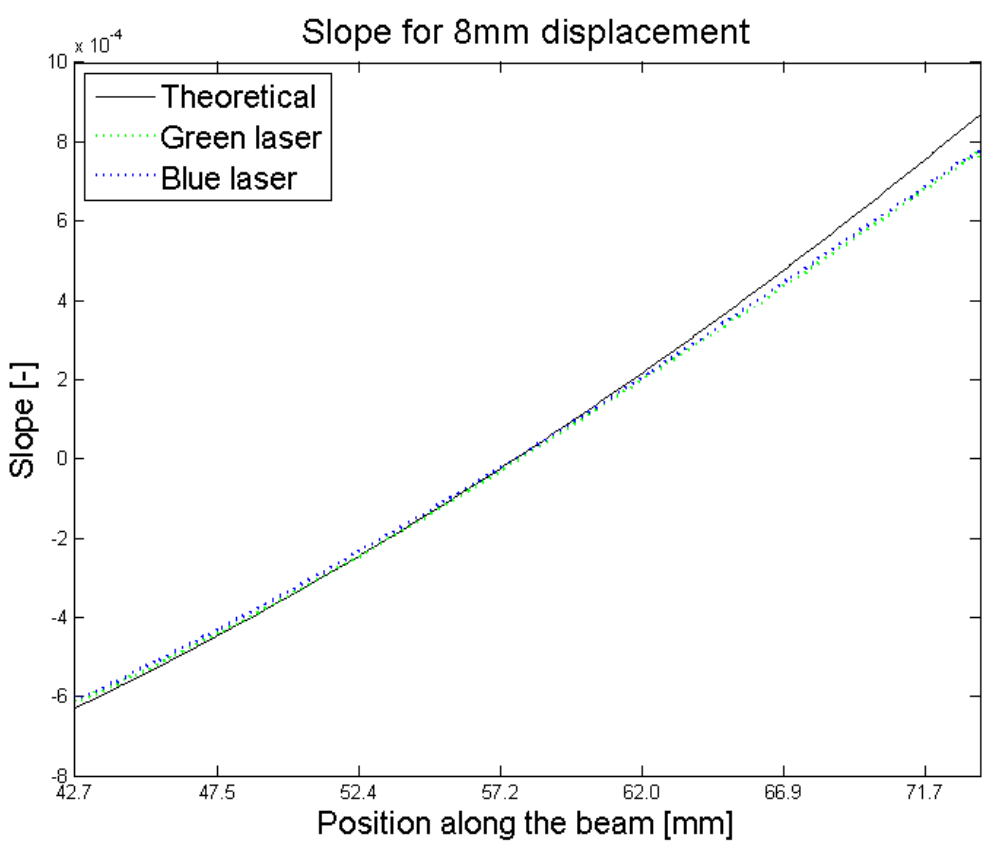

Figure 43 - Relative slope of blue and green laser for an 8mm imposed displacement.

Table 6 summarizes the relative surface slope errors for a sequence of $8 \times 1 \mathrm{~mm}$ displacement imposed on the beam. From $1 \mathrm{~mm}$ to $5 \mathrm{~mm}$ imposed displacement on the beam, the correction algorithm is effective in producing acceptable results even if the deliberate misalignment is large. From $6 \mathrm{~mm}$ imposed displacement, the quality of the results is degrading thus showing a limit to that correction procedure.

Table 6 - Absolute errors on the relative slope measured with green and blue.

\begin{tabular}{|c|c|c|c|c|c|c|c|c|c|}
\cline { 3 - 9 } \multicolumn{2}{c|}{} & $\begin{array}{c}1 \mathrm{~mm} \\
{[\mu \text { strain }]}\end{array}$ & $\begin{array}{c}2 \mathrm{~mm} \\
{[\mu \text { strain }]}\end{array}$ & $\begin{array}{c}3 \mathrm{~mm} \\
{[\mu \mathrm{strain}]}\end{array}$ & $\begin{array}{c}4 \mathrm{~mm} \\
{[\mu \mathrm{strain}]}\end{array}$ & $\begin{array}{c}5 \mathrm{~mm} \\
{[\mu \text { strain }]}\end{array}$ & $\begin{array}{c}6 \mathrm{~mm} \\
{[\mu \mathrm{strain}]}\end{array}$ & $\begin{array}{c}7 \mathrm{~mm} \\
{[\mu \text { strain }]}\end{array}$ & $\begin{array}{c}8 \mathrm{~mm} \\
{[\mu \mathrm{strain}]}\end{array}$ \\
\hline \multirow{2}{*}{ blue } & MAE & 2.3 & 8.5 & 7.2 & 8.6 & 13.6 & 12.0 & 16.9 & 22.1 \\
\cline { 2 - 10 } & Max AE & 10.0 & 30.9 & 35.8 & 35.2 & 49.6 & 70.9 & 78.7 & 105.4 \\
\hline \multirow{2}{*}{ green } & MAE & 3.7 & 6.5 & 3.5 & 5.1 & 11.9 & 18.2 & 18.2 & 23.3 \\
\cline { 2 - 10 } & Max AE & 7.4 & 28.2 & 36.7 & 44.2 & 57.2 & 99.9 & 90.6 & 116.5 \\
\hline
\end{tabular}

The limit of the correction for non-uniform shear is more obvious in Figure 44(b) which depicts the absolute phase determination using blue and green signals for an $8 \mathrm{~mm}$ imposed displacement. For this experiment, the mean absolute error jumps to more than $77 \%$. The results are not so dramatic for the $4 \mathrm{~mm}$ imposed displacement case, where the most part remains exploitable as represented in Figure 44(a). 


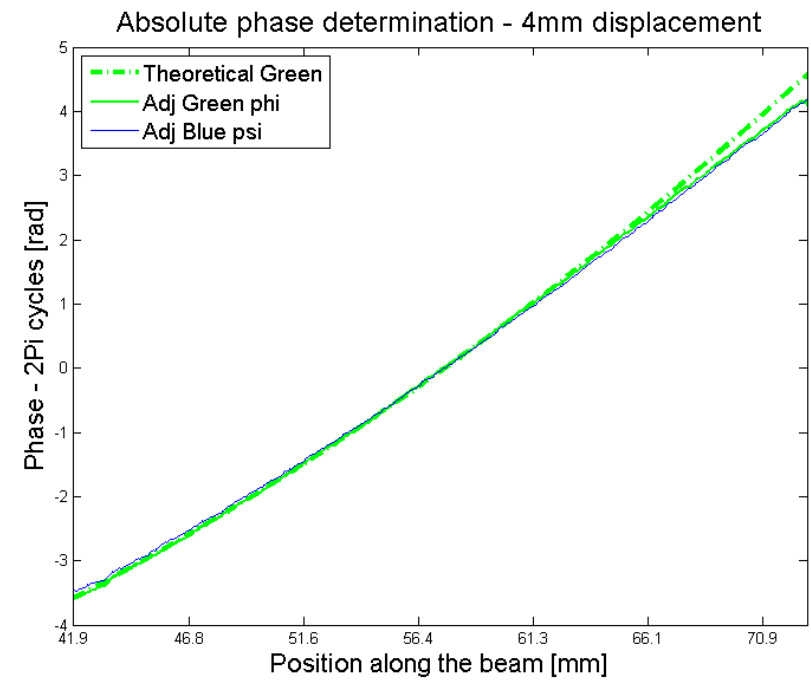

(a)

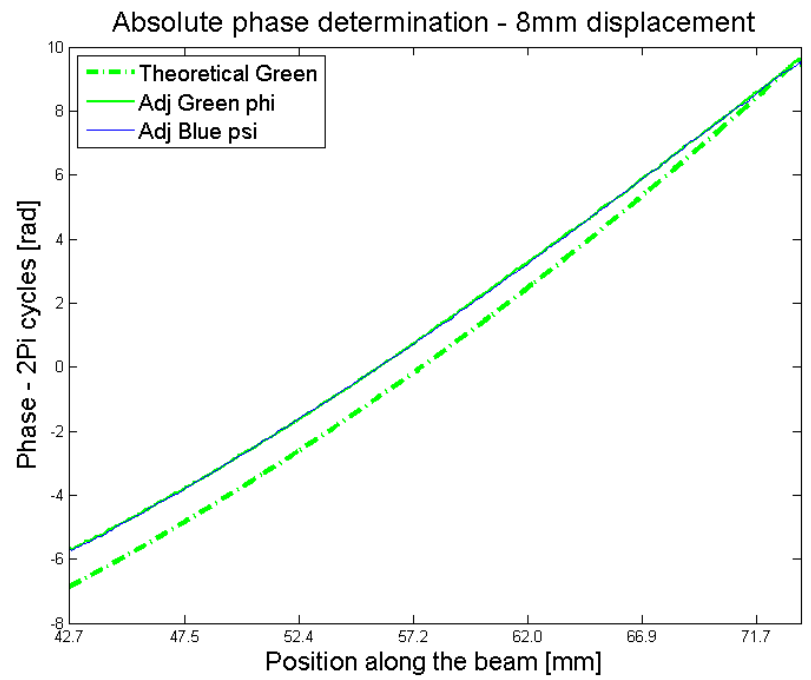

(b)

Figure 44 - Absolute phase determination for (a) $4 \mathrm{~mm}$ and (b) $8 \mathrm{~mm}$ imposed displacement.

As for the relative surface slope measurement, the absolute phase determination confirms the limit of the non-uniform shear correction algorithm. The errors are summarized in Table 7, where a significant jump on the error values between 5 and $6 \mathrm{~mm}$ imposed displacement can be seen. From $6 \mathrm{~mm}$ imposed displacement, the calculated absolute phase values cannot be used for absolute phase interpretation anymore.

Table 7 - Absolute errors on the absolute green phase measured with green and blue.

\begin{tabular}{|c|c|c|c|c|c|c|c|c|c|}
\cline { 2 - 10 } \multicolumn{2}{c|}{} & $\begin{array}{c}1 \mathrm{~mm} \\
{[2 \pi \text { cycles }]}\end{array}$ & $\begin{array}{c}2 \mathrm{~mm} \\
{[2 \pi \text { cycles }]}\end{array}$ & $\begin{array}{c}3 \mathrm{~mm} \\
{[2 \pi \text { cycles }]}\end{array}$ & $\begin{array}{c}4 \mathrm{~mm} \\
{[2 \pi \text { cycles }]}\end{array}$ & $\begin{array}{c}5 \mathrm{~mm} \\
{[2 \pi \text { cycles }]}\end{array}$ & $\begin{array}{c}6 \mathrm{~mm} \\
{[2 \pi \text { cycles }]}\end{array}$ & $\begin{array}{c}7 \mathrm{~mm} \\
{[2 \pi \text { cycles }]}\end{array}$ & $\begin{array}{c}8 \mathrm{~mm} \\
{[2 \pi \text { cycles }]}\end{array}$ \\
\hline \multirow{2}{*}{ blue } & MAE & 0.03 & 0.09 & 0.08 & 0.09 & 0.15 & 0.80 & 0.81 & 0.77 \\
\cline { 2 - 11 } & Max AE & 0.11 & 0.34 & 0.39 & 0.39 & 0.55 & 1.03 & 1.12 & 1.22 \\
\hline \multirow{2}{*}{ green } & MAE & 0.04 & 0.07 & 0.04 & 0.06 & 0.13 & 0.80 & 0.81 & 0.78 \\
\cline { 2 - 10 } & Max AE & 0.08 & 0.31 & 0.40 & 0.49 & 0.63 & 1.01 & 1.11 & 1.21 \\
\hline
\end{tabular}

For the surface shape reconstruction, the results look more stable than for the absolute phase determination. In fact displacement results obtained with wanted misalignment remain relatively similar to the results measured without misalignment, using correction for non-uniform shear for both cases. The displacement values summarized in

Table 8 show comparable results with Table 5. The correction is efficient at some extent but this large introduced misalignment affects the shape reconstruction by adding noise. The fringe patterns shown in Figure 45, which represent the wrapped reconstructed phase maps before being unwrapped and converted to displacement values, have clearly a difference in their respective noise. 


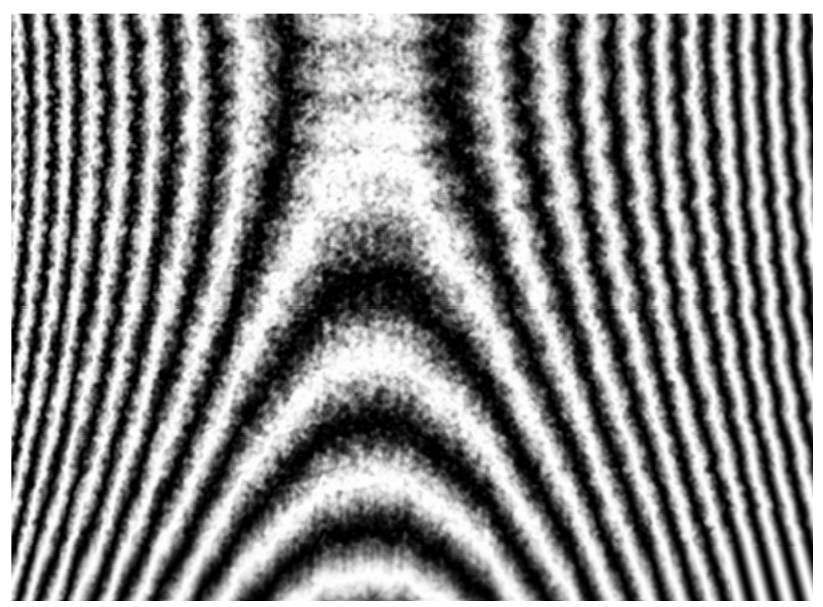

(a)

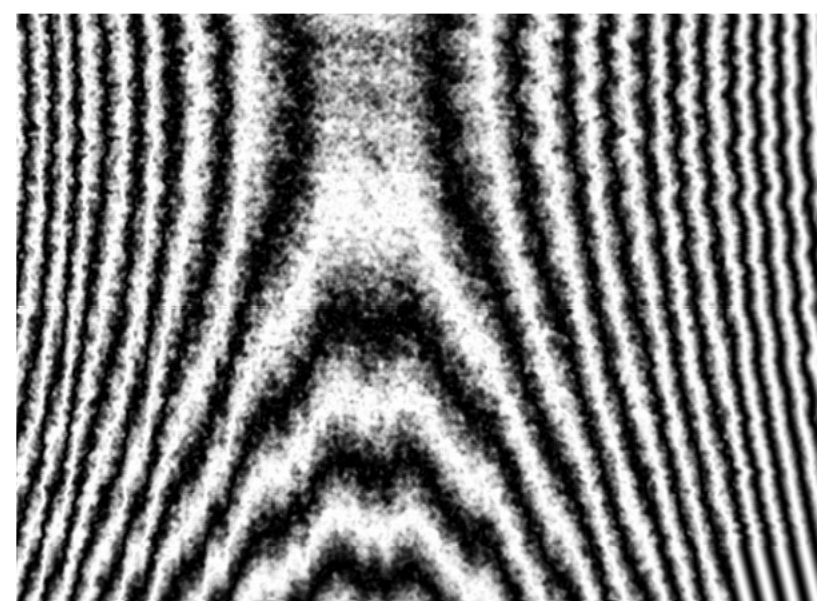

(b)

Figure 45 - Wrapped reconstructed phase maps for surface displacement shape reconstruction for $4 \mathrm{~mm}$ imposed displacement with correction for non-uniform shear (a) wrapped phase map without mirror misalignment (b) wrapped phase map with mirror misalignment.

Table 8 - Absolute errors on the measured surface displacement shape with green and red.

\begin{tabular}{|c|c|c|c|c|c|c|c|c|c|}
\cline { 2 - 9 } \multicolumn{2}{c|}{} & $\begin{array}{c}1 \mathrm{~mm} \\
{[\mu \mathrm{m}]}\end{array}$ & $\begin{array}{c}2 \mathrm{~mm} \\
{[\mu \mathrm{m}]}\end{array}$ & $\begin{array}{c}3 \mathrm{~mm} \\
{[\mu \mathrm{m}]}\end{array}$ & $\begin{array}{c}4 \mathrm{~mm} \\
{[\mu \mathrm{m}]}\end{array}$ & $\begin{array}{c}5 \mathrm{~mm} \\
{[\mu \mathrm{m}]}\end{array}$ & $\begin{array}{c}6 \mathrm{~mm} \\
{[\mu \mathrm{m}]}\end{array}$ & $\begin{array}{c}7 \mathrm{~mm} \\
{[\mu \mathrm{m}]}\end{array}$ & $\begin{array}{c}8 \mathrm{~mm} \\
{[\mu \mathrm{m}]}\end{array}$ \\
\hline \multirow{2}{*}{ shape } & MAE & 0.06 & 0.11 & 0.19 & 0.22 & 0.27 & 0.35 & 0.43 & 0.49 \\
\cline { 2 - 11 } & Max AE & 0.16 & 0.25 & 0.47 & 0.67 & 0.73 & 1.00 & 1.20 & 1.40 \\
\hline
\end{tabular}

\subsection{Discussion}

Simultaneous ESPI measurements using multiple wavelengths and a color camera is shown to be both practical and effective. The fringe patterns in Figure 36 from the single-axis shearography arrangement in Figure 19 show good contrast. The fringe patterns in Figure 37 from the two-axis shearography arrangement in Figure 22 are slightly noisier. However, this additional noise does not seem to affect the quantitative measurements and it is not an artifact of the use of multiple colors because the single-axis shearography results are clean. Although not fully investigated during this research, part of the reason may be that each added optical component, in this case the cyan dichroic filter, reduces wavefront quality.

The use of two colors enables simultaneous, full-field and non-contact measurements of two surface slopes. Here, green and red laser lights are used to retrieve the surface slopes $\partial w / \partial x$ and $\partial w / \partial y$. Results shown in Figure 37, Figure 38, and Table 3 are really promising. This new compact two-axis sheargaphy coupled with the use of multiple wavelengths and a color camera as a great potential in the industry. 
The example use of multiple color measurements described here uses two of the three available colors for relative surface slope determination, leaving the third color (blue here) available for further use. A practical application for the third color is the determination of the absolute phase on one of the shear measurements, a feature that is typically not available when unwrapping ESPI phase maps because of the associated modulo $2 \pi$ ambiguity. The two-wavelength method $[95,96]$ is applied for absolute phase identification, for example using blue and green light in this case. The presented results in Figure 39 and Table 4, measured and calculated following the two-wavelength method, are in good agreement with the theoretical values, with a mean absolute percentage error remaining under $12 \%$, thus validating the twowavelength method for retrieving absolute phase. For this to be done even more effectively, the wavelength difference between the two colors must be small, rather smaller than with the presently available RGB light sources, so as to extend the measurement range given by the wavelength ratio.

The surface slopes $\partial w / \partial x$ and $\partial w / \partial y$, obtained using green and red laser light, are summed to determine the surface displacement shape. Given the well-known stability of shearography measurements, this could provide a robust displacement measurement technique that is much less delicate than typical ESPI measurements. The horizontal fringes shown in Figure 37(c) were thought to be only the representation of the anticlastic curvature of the beam. Figure $40(\mathrm{a}-\mathrm{b})$ illustrate both a displacement gradient with respect to the $y$-direction which is not symmetric and which is non-symmetrically increasing while the imposed displacement is augmented. The beam is deflected at one of its extremity via the micrometer. The other extremity is constrained by a cone-point set screw. The overhanging beam pivots around a shoulder screw. One hypothesis is that the shoulder screw slightly bends while the beam is bent by the micrometer. Another hypothesis concerns the fact that the cone point set screw is not perfectly aligned with middle of the beam looking at the $y$-direction. The displacement gradient with respect to the $y$ direction is a mixture of some anticlastic curvature and both hypothesises previously mentioned. The theoretical model, by mean of the physical overhanging beam assembly, has some difficulty to make the measurements match the theoretical values issued from the beam theory but the presented results clearly demonstrate that reconstruction or the deformed shape is working and that with a mean absolute error oscillating between $0.07 \mu \mathrm{m}$ and $0.49 \mu \mathrm{m}$ over eight different measurements.

The correction algorithm for non-uniform shear was first developed to counter the non-flatness of the thin cyan dichroic filter used in the two-axis shearography arrangement. The non-flatness of that filter was discovered by analyzing the shear distance with DIC. The algorithm does not only correct the measured phase for mirror non-flatness but it also provides an efficient mathematical adjustment for mirror misalignment. The correction remains very effective for small shear non-uniformities but larger unwanted defaults cause the algorithm to fail. This phenomenon is clear when using the corrected phase maps for retrieving the absolute phase maps while the shape reconstruction stays relatively insensitive. 


\section{Chapter 6. Conclusions and Future Work}

\subsection{Contribution}

The use of a color camera for doing simultaneous ESPI measurements has been demonstrated. This capability is possible by using the three separate color measurements available with a Bayer style camera. The technique is valuable because it allows the use of much simpler and more compact optical equipment and because it provides multiple independent measurements that are automatically coordinated in both time and space.

An example of multiple simultaneous ESPI measurements has been demonstrated here using a standard single-axis shearography apparatus and independent ESPI fringe patterns were obtained in two different colors. Further example measurements were made using a two-axis shearography apparatus designed to measure surface gradients in two directions. That two-axis shearographic interferometer is a unique design that combines two modified Michelson interferometers in one by simply adding one extra component. Inserting a single dichroic filter in one of the interferometer arm, between the already existing mirror and beamsplitter cube, allows simultaneous acquisition of two pairs of sheared images having the same spatial reference. The dichroic filter acts as a reference while the two other tilt mirrors must be oriented to get the desired shear distance and direction. In addition to the compact double interferometer, measuring slopes in two different directions implies to illuminate the object surface with two laser lights of different wavelengths. The use of colors was combined with the novel two-axis shearographic interferometer to measure slopes on an overhanging beam. This produced clearly identifiable vertical and horizontal fringes, respectively corresponding to the surface slopes resultant from longitudinal bending and anticlastic curvature. Some cross-talk exists between the raw measurements when using a typical Bayer style camera. Fortunately, it can the largely eliminated using a simple mathematical adjustment, thus producing three independent measurements.

With sheorography, the measurement sensitivity is driven by the optical geometry configuration, the wavelength involved in the measurement, and the applied shear distance. The measurement accuracy is directly dependent on these three factors, which need to be accurately known. A convenient method to accurately measure the shear between two single images is to use $2 \mathrm{D}$ digital image correlation (DIC) to identify the non-uniform shear produced by the two-axis interferometer. The surface slope being measured in two orthogonal directions allows use of a mathematical procedure to correct for non-uniform shears and small mirror misalignments.

Shearography measures the finite difference between the two optical paths through the interferometer. This approximately corresponds to the out-of-plane surface slope. The measured surface slopes were quantitatively compared with theoretical values working with an overhanging beam setup. The beam, instead of being loaded with calibrated weights, was bent with known displacements through a 
micrometer, thus removing the need to know the beam material Young's modulus precisely when determining the theoretical surface slopes. All measurements were in good agreement with the theoretically expected values, typically within $3 \%$. The results confirm that the multi-color technique is an effective tool for quantitative measurements. Use of multiple wavelengths and a color camera is not limited to shearography but opens the doors to simultaneous multi-measurement for most optical configurations of the ESPI family.

Two different colors, green and red here, were used for measuring relative slopes, thus leaving a channel free on the Bayer style camera. The third available color, blue, was used redundantly with green light to enable use of the two-wavelength method to determine absolute slope independent of the boundary conditions. For simple case where zero boundary conditions are contained within the recorded images, it is possible to extract the zero fringe order. However, in most cases encountered in the industry need a technique, like the two-wavelength method, to extract the absolute phase, which is proportional to the absolute surface slope when using shearography. The two-wavelength method, applied with a green laser, $532 \mathrm{~nm}$ wavelength, and a blue laser, $473 \mathrm{~nm}$ wavelength, was proven to be effective and matching reasonably well the theoretical values.

Having phase maps in two orthogonal directions offers the possibility to retrieve the deformed shape of test specimen. Shearography is mainly sensitive to out-of-plane deformations induced by surface rotations, according to the geometrical configuration chosen, and measures the out-of-plane component relative to the finite shear distance. The measured quantity is an approximation to the out-of-plane displacement derivative and the measured phase values can be summed, with respect to the shear directions, and converted to displacement to yield the deformed shape. Working with a green laser, $532 \mathrm{~nm}$ wavelength, coupled with $\mathrm{x}$-shear on the two-axis interferometer and a red laser, 660nm wavelength, coupled with a $y$-shear, provides the two phase maps necessary for calculating the displacement field. The reconstructed displacement field, obtained from a summation procedure of green and red phase maps, of an overhanging beam, bent through controlled displacements, was measured within a mean accuracy of $\pm 0.49 \mu \mathrm{m}$ over eight different measurements. The associated absolute displacements were in the range 6 to $54 \mu \mathrm{m}$. The slope, absolute phase, and deformed shape measurements were repeated with intentional misalignment of one of the mirrors to show the effectiveness of the correction for non-uniform shear. The correction algorithm, which adjusts for mirror non-flatness and mirror misalignment, is effective and gives acceptable results for the slope measurements. The two-wavelength method, developed for retrieving absolute phase, was mostly but not always successful. For the deformed shape measurement, the calculation remains stable, however results get slightly noisier. 


\subsection{Remaining Challenges and Limitations}

A general limitation of the ESPI method is the measurement range. Deformations in the micron scale can be measured with standard systems. With larger deformations, the number of fringes becomes too big to be resolvable. The measurement range is mainly limited by the measurement sensitivity defined by the wavelength of the laser, the angle of illumination, as well as the shear distance for Shearography. The use of larger wavelengths could help measuring larger deformations. Furthermore, a longer wavelength would decrease the Signal-to-Noise Ratio (SNR) and lower the effect of external disturbances, such as external vibrations, on measurements. Subtracting two different wavelengths measuring the exact same deformation would have the same effect than using a larger wavelength.

The innovative two-axis shearographic interferometer developed and built during this research is limited to measure absolute phase in only one direction. To get the absolute phase in two different directions using the two-wavelength method would imply a fourth color to illuminate the object. Adapting the laser source would not be the only change to make on the interferometric arrangement. A camera capable of capturing four independent signals is needed. Typical RGB cameras measure only three independent signals. Four-color cameras containing a CYGM sensor (Cyan, Yellow, Green, Magenta) do exist and could satisfy the need.

During experimentation, an area of $31 \times 23 \mathrm{~mm}$ was inspected. The RGB source of laser light is collimated to create a constant sensitivity vector across the region of interest. The collimation, providing here a $50 \mathrm{~mm}$ diameter beam, is dependent of the laser source, magnification, and the plano-convex lens used. For inspection of larger areas, more powerful lasers would be required, as well as larger magnification and collimation lenses, which may not always be practical. It is easier to have non-collimated light for measuring large surfaces, but quantitative measurements require mathematical corrections to account for the non-constancy of the sensitivity vector.

The effective range of calculation of the absolute phase is limited by the wavelength ratio. The range could be enlarged by choosing closer wavelengths with the risk of being unable to identify and separate them. The two-wavelength method is effective for small deformations but rather sensitive to noise. The goal of this research was not to focus on developing an absolute measurement immune to any noise and applicable for any situations. The goal was to implement the use of colors and a single color camera for doing interferometry. The absolute phase measurement using the two-wavelength method might not be the most effective method for obtaining absolute phase but it is a good example of what can be accomplished with multiple colors.

The correction for non-uniform shear is limited to small shear distortions. Two main sources of distortions were identified. Mirror misalignment and mirror non-flatness cause the shear to be uneven across the image. The effective range of the correction algorithm needs to be defined more precisely. In this study, 
an example is shown to prove the effectiveness for larger shear distortion where one of the mirrors was voluntarily misaligned. The algorithm was developed to counter the non-flatness of the thin dichroic filter, which produced unwanted non-uniform shear. The improvement brought by the correction was not quantified.

Figure 37 (a) and (b) show interference fringes obtained while bending the overhanging beam used for experimentation. It appears that the largest deformation, located between the shoulder screw acting like a pivot and the cone point set screw, is related to a zero slope and spatially corresponds to the middle of the fringe pattern. The contrast between a white and a dark fringe is higher in the middle of the image from top to bottom and slowly decreases going to the right or left of the image. The fringes get noisier when moving from the zero slope location suggesting that pixel decorrelation occurs, which was measured using DIC. The fringe pattern shows a decorrelation of 0.15 pixel on both edges but a so small decorrelation should not have a such effect on the fringe quality. This phenomenon needs to be studied more in detail.

The novel two-axis shearographic interferometer is composed of two modified Michelson interferometer combined into one unit. Both interferometers have unequal arm lengths because of the asymmetry brought by the added dichroic filter. The macro lens was almost fully zoomed to get the beam's height occupying the entire camera frame. Working with the lens totally open and zoomed at its maximum was making difficult to get the object plane in focus because of the short depth of focus of the macro lens. When recording speckle patterns, the lens was set to a F-number of 8 which increased the depth of focus. Any large difference in the optical path, for each interferometer, between the object and the sensor plane combined with a short depth of focus would result in blurring the pair of sheared images. The influence of unequal interferometer arm lengths needs to be analyzed more deeply.

When reading the raw color signals, the missing two color values at each pixel must be estimated due to the Bayer pattern design. The demosaicing process consists here in a linear interpolation of the known color values from the nearest neighboring pixel. More evolved interpolations for demosaicing are available such as: cubic interpolation, gradient-corrected bilinear interpolation, smooth hue transition interpolation, pattern recognition interpolation, adaptive color plane interpolation, or directionally weighted gradient based interpolation. None of the previously cited interpolations were tested. The different interpolation procedures, for recovering the missing pixel values, should be explored to determine which one is the most effective.

Furthermore, working with a Bayer type camera raises the question for optimum speckle size since spaces are found between pixels. With a monochromatic camera, the speckle size should corresponds to the pixel size of the camera sensor or be greater to guaranty maximum intensity variations on pixels. Theoretically, speckles of larger size could be used with a Bayer filter and produced by closing the lens 
aperture slightly more. That statement has not been verified yet but the optimum speckle size is certainly changed while using a Bayer type camera and it necessitates further investigations.

Finally, the overhanging beam experiment allows to quantitatively compare the measured values with the expected theoretical values but in only one direction. A cylindrical, square, or rectangular membrane would permit to validate qualitative measurements in two orthogonal directions. Shape functions for the three different cases are known and theoretical values are "easily" computed. The membrane has to be designed such that the maximum deformation remains within the measurable range of the interferometer and that the pressure, to reach the maximum deformation, is high enough to guaranty a minimum pressure adjustment sensitivity. Concerning the overhanging beam system, all non-standard parts were machined in the machine shop of the Mechanical department of UBC. The system was designed to measure out-of-plane surface slopes on a tight toleranced beam in bending but the final product does not match perfectly the theory. In fact, every imperfection drives the matching with theoretical values away suggesting that the overhanging beam system should be calibrated with another measurement method, which has itself been calibrated.

\subsection{Future Work and Recommendations}

ESPI has already been combined with shearography. Kim and Gweon developed and tested a system that can observe in-plane, out-of-plane displacement and defect on the surface using ESPI and shearography [103]. The main disadvantage of their optical arrangement is that each different quantity has to be measured sequentially. Implementing the use of multiple colored sources and a color camera could greatly improve the functioning of their particular interferometer.

The advantage of using simultaneously different wavelengths and a color could be taken for doing inplane ESPI measurements in three different directions. An optical arrangement with three measurement sensitivities corresponds to an optical strain rosette. Based on the idea of a strain gauge rosette, which measures normal strains along two different directions and also the shear strain, an optical strain rosette would have the advantage of being a non-contact, nondestructive, and full-field measurement technique. 


\section{Bibliography}

[1] D. Gabor, "Holography, Past, Present And Future", SPIE Proceedings, Vol. 25, Developments in Holography II, pp. 129-136, 1971.

[2] J. M. Burch, "Laser Speckle Metrology", SPIE Proceedings, Vol. 25, Developments in Holography II, pp. 149-156, 1971.

[3] K. A. Stetson, "A Review of Speckle Photography and Interferometry", Opt. Eng. 14(5), 145482, p. 145482, Oct. 1975.

[4] J. A. Leendertz, "Interferometric displacement measurement on scattering surfaces utilizing speckle effect", J. Phys. E: Sci. Instrum. 3, pp. 214-218, Mar. 1970.

[5] J. N. Butters and J. A. Leendertz, "Speckle pattern and holographic techniques in engineering metrology", Optics and Laser Technology, 3(1), pp. 26-30, Feb. 1971.

[6] W. Steinchen and L. Yang, Digital Shearography: Theory and Application of Digital Speckle Pattern Shearing Interferometry, Society of Photo Optical, p. 310, 2003.

[7] E. K. Hack and M. Riner, "3D ESPI and 3D shearography measurements applied to NDT and FEM analysis validation for industrial quality control", SPIE Proceedings 4398, Optical Measurement Systems for Industrial Inspection II: Application in Industrial Design, pp. 155-167, 2001.

[8] M. Burnett and P. J. Bryanston-Cross, "Measurements of transonic shock structures using shearography", SPIE Proceedings 2861, Laser Interferometry VIII, pp. 124-135, 1996.

[9] A. Macovski, S. D. Ramsey, and L. F. Schaefer, "Time-lapse interferometry and contouring using television systems.", Applied Optics, Vol. 10, Issue 12, pp. 2722-7, Dec. 1971.

[10] J. N. Butters, "Some applications of electronic speckle pattern interferometry", International Optical Computing Conference, Washington, D.C, Digest of Papers. (A75-41476 20-60) New York, Institute of Electrical and Electronics Engineers, pp. 85-89, 1975.

[11] A. E. Ennos, "Speckle interferometry", Laser Speckle and Related Phenomena Topics in Applied Physics Volume 9, pp. 203-253, 1975.

[12] D. Denby, J. N. Butters, and G. E. Quintanilla, "Contouring by electronic speckle pattern interferometry", (A77-20576 07-35) Cambridge University Press, pp. 171-197, 1976.

[13] T. J. Cookson, J. N. Butters, and H. C. Pollard, "Pulsed lasers in electronic speckle pattern interferometry", Optics \& Laser Technology Volume 10, Issue 3, pp. 119-124, Jun. 1978.

[14] O. J. Løkberg and K. Høgmoen, "Vibration phase mapping using electronic speckle pattern interferometry.", Applied Optics, Vol. 15, Issue 11, pp. 2701-4, Nov. 1976.

[15] G. A. Slettemoen, "Electronic speckle pattern interferometric system based on a speckle reference beam.", Applied Optics, Vol. 19, Issue 4, pp. 616-23, Feb. 1980.

[16] O. J. Løkberg, "Use of chopped laser light in electronic speckle pattern interferometry.", Applied Optics, Vol. 18, Issue 14, pp. 2377-84, Jul. 1979. 
[17] O. J. Løkberg, K. Høgmoen, and O. M. Holje, "Vibration measurement on the human ear drum in vivo.", Applied Optics, Vol. 18, Issue 6, pp. 763-5, Mar. 1979.

[18] O. J. Løkberg and K. Krakhella, "Electronic speckle pattern interferometry using optical fibers", Optics Communications Vol. 38, Issue 3, pp. 155-158, Aug. 1981.

[19] O. J. Løkberg and O. Kwon, "Electronic speckle pattern interferometry using a CO2 laser", Optics \& Laser Technology Vol. 16, Issue 4, pp. 187-192, Aug. 1984.

[20] O. J. Lokberg, "Long-Distance Electronic Speckle Pattern Interferometry", Opt. Eng. 27(2), p. 272150, Feb. 1988.

[21] S. Nakadate, T. Yatagai, and H. Saito, "Electronic speckle pattern interferometry using digital image processing techniques.", Applied Optics, Vol. 19, Issue 11, pp. 1879-83, Jun. 1980.

[22] S. Nakadate, T. Yatagai, and H. Saito, "Computer-Aided Speckle Pattern Interferometry", SPIE Proceedings, Vol. 0370, pp. 180-188, 1983.

[23] S. Nakadate and H. Saito, "Fringe scanning speckle-pattern interferometry", Applied Optics, Vol. 24, Issue 14, p. 2172, Jul. 1985.

[24] K. Creath, "Digital Speckle Pattern Interferometry (DSPI) Using A 100X100 Imaging Array", SPIE Proceedings, Vol. 0501, pp. 292-298, 1984.

[25] K. Creath, "Phase-Shifting Speckle Interferometry", SPIE Proceedings, Vol. 0556, pp. 337-346, 1985.

[26] K. Creath, "Interferometric investigation of a diode laser source", Applied Optics, Vol. 24, Issue 9, p. 1291, 1985.

[27] D. Kerr, F. M. Santoyo, and J. R. Tyrer, "Manipulation of the Fourier Components of Speckle Fringe Patterns as Part of an Interferometric Analysis Process", Journal of Modern Optics Vol. 36, Issue 2, pp. 195-203, Feb. 1989.

[28] A. J. Moore and J. R. Tyrer, "An electronic speckle pattern interferometer for complete in-plane displacement measurement", Meas. Sci. Technol. 1, pp. 1024-1030, Oct. 1990.

[29] M. Owner-Petersen, "Decorrelation and fringe visibility: on the limiting behavior of various electronic speckle-pattern correlation interferometers", JOSA A, Vol. 8, Issue 7, p. 1082, 1991.

[30] N. K. Mohan, H. Saldner, and N.-E. Molin, "Electronic speckle pattern interferometry for simultaneous measurement of out-of-plane displacement and slope", Optics Letters, Vol. 18, Issue 21, p. 1861, Nov. 1993.

[31] G. Pedrini, B. Pfister, and H. Tiziani, "Double Pulse-electronic Speckle Interferometry", Journal of Modern Optics Vol. 40, Issue 1, pp. 89-96, Jan. 1993.

[32] A. J. Haasteren and H. J. Frankena, "Real-time displacement measurement using a multicamera phase-stepping speckle interferometer.", Applied Optics, Vol. 33, Issue 19, pp. 4137-42, Jul. 1994. 
[33] C. K. Hong, H. S. Ryu, and H. C. Lim, "Least-squares fitting of the phase map obtained in phaseshifting electronic speckle pattern interferometry", Optics Letters, Vol. 20, Issue 8, p. 931, Apr. 1995.

[34] C. Joenathan, B. Franze, P. Haible, and H. J. Tiziani, "Speckle Interferometry with Temporal Phase Evaluation for Measuring Large-Object Deformation", Applied Optics, Vol. 37, Issue 13, p. 2608, May 1998.

[35] G. S. Schajer and M. Steinzig, "Full-field calculation of hole drilling residual stresses from electronic speckle pattern interferometry data", Experimental Mechanics, Vol. 45, Issue 6, pp. 526-532, Dec. 2005.

[36] H. A. Aebischer and S. Waldner, "A simple and effective method for filtering speckleinterferometric phase fringe patterns", Optics Communications Vol. 162, Issues 4-6, pp. 205-210, Apr. 1999.

[37] M. A. Gdeisat, D. R. Burton, and M. J. Lalor, "Spatial carrier fringe pattern demodulation by use of a two-dimensional continuous wavelet transform", Applied Optics, Vol. 45, Issue 34, p. 8722, Dec. 2006.

[38] Q. Kemao, "Two-dimensional windowed Fourier transform for fringe pattern analysis: Principles, applications and implementations", Optics and Lasers in Engineering Vol. 45, Issue 2, pp. 304317, Feb. 2007.

[39] A. Huang and J. Peng, "Test verification for improved design of rotor blade by ESPI", SPIE Proceedings, Vol. 7375, Distribution Analysis of Displacements and Strains, p. 73753T-73753T-4, 2008.

[40] M. Morschel and G. Bastian, "Interferometric Analysis of Thermomechanical Deformations in Thermoelectric Generators", Journal of Electronic Materials, Vol. 42, Issue 7, pp. 1669-1675, 2012.

[41] A. W. Koch, A. Purde, and M. Jakobi, "Contouring of surfaces with discontinuities using ESPI", SPIE Proceedings, Vol. 6341, pp. 634126-634126-6, 2006.

[42] M. Hertwig, "Application of improved Speckle contouring technique to surface roughness measurements", pp. 115-130, Jan. 1997.

[43] L. Yang, P. Zhang, S. Liu, P. R. Samala, M. Su, and H. Yokota, "Measurement of Strain Distributions in Mouse Femora with 3D-Digital Speckle Pattern Interferometry.", Optics and Lasers in Engineering Vol. 45, Issue 8, pp. 843-851, Aug. 2007.

[44] B. P. HILDEBRAND and K. A. HAINES, "Multiple-Wavelength and Multiple-Source Holography Applied to Contour Generation", JOSA, Vol. 57, Issue 2, p. 155, Feb. 1967.

[45] B. E. Bayer, "Color imaging array", US3971065 A, 20-Jul-1976.

[46] E. Hack and R.P. Kaestle, "Multi-wavelengths shearography for optical whole-field strain measurements", Colloque Nouveaux Moyens Optiques Pour L'Industrie II, Mittelwihr/Colmar (F), Proceedings, pp. 31-36, 1999.

[47] P. Georgas, "Simultaneous measurement of full-field vibration modes using Electronic Speckle Pattern Interferometry (ESPI)", University of British Columbia, 2012. 
[48] J. A. Leendertz and J. N. Butters, "An image-shearing speckle-pattern interferometer for measuring bending moments", J. Phys. E: Sci. Instrum. 6, pp. 1107-1110, Nov. 1973.

[49] Y. Y. Hung and R. M. Grant, "Shearography: A New Optical Method for Nondestructive Evaluation of Tires", Rubber Chemistry and Technology, Vol. 54, No. 5, pp. 1042-1050, Nov. 1981.

[50] Y. Y. Hung, "A speckle-shearing interferometer: A tool for measuring derivatives of surface displacements", Optics Communications, Vol. 11, Issue 2, pp. 132-135, Jun. 1974.

[51] Y. Y. Hung and C. E. Taylor, "Speckle-Shearing Interferometric Camera -- A Tool For Measurement Of Derivatives Of Surface-Displacement", SPIE Proceedings, Vol. 0041, pp. 169_ 176, 1974.

[52] Y. Y. Hung, R. E. Rowlands, and I. M. Daniel, "Speckle-shearing interferometric technique: a fullfield strain gauge.", Applied Optics, Vol. 14, Issue 3, pp. 618-22, Mar. 1975.

[53] Y. Y. Hung and C. Y. Liang, "Image-shearing camera for direct measurement of surface strains.", Applied Optics, Vol. 18, Issue 7, pp. 1046-51, Apr. 1979.

[54] Y. Y. HUNG and A. J. DURELLI, "Simultaneous measurement of three displacement derivatives using a multiple image-shearing interferometric camera", The Journal of Strain Analysis for Engineering Design, vol. 14, no. 3, pp. 81-88, Jul. 1979.

[55] Y. Y. Hung, "Shearography: A New Optical Method For Strain Measurement And Nondestructive Testing", Optical Engineering, Vol. 21, Issue 3, p. 213391, 1982.

[56] J. Takezaki and Y. Y. Hung, "Direct Measurement of Flexural Strains in Plates by Shearography", Journal of Applied Mechanics, Vol. 53, Issue 1, p. 125, Mar. 1986.

[57] Y. Y. Hung, J. D. Hovanesian, and J. Takezaki, "A fringe carrier technique for unambiguous determination of fringe orders in shearography", Optics and Lasers in Engineering, Vol. 8, Issue 2, pp. 73-81, Jan. 1988.

[58] H. M. Shang, "Surface profiling using shearography", Optical Engineering, Vol. 39, Issue 1, p. 23, Jan. 2000.

[59] D. DENBY and J. A. LEENDERTZ, "Plane-surface strain examination by speckle-pattern interferometry using electronic processing", The Journal of Strain Analysis for Engineering Design, vol. 9, no. 1, pp. 17-25, Jan. 1974.

[60] P. Hariharan, "Speckle-shearing interferometry: a simple optical system.", Applied Optics, Vol. 14, Issue 11, p. 2563, Nov. 1975.

[61] S. Debrus, "Speckle shearing interferometer using a Savart plate", Optics Communications, Vol. 20, Issue 2, pp. 257-261, Feb. 1977.

[62] A. Assa, A. A. Betser, and J. Politch, "Recording slope and curvature contours of flexed plates using a grating shearing interferometer.", Applied Optics, Vol. 16, Issue 9, pp. 2504-13, Sep. 1977.

[63] S. Nakadate, T. Yatagai, and H. Saito, "Digital speckle-pattern shearing interferometry.", Applied Optics, Vol. 19, Issue 24, pp. 4241-6, Dec. 1980. 
[64] Y. J. Chao, M. A. Sutton, and C. E. Taylor, "A simple tool for speckle-shearing interferometry", Experimental Mechanics, Vol. 21, Issue 11, pp. 436-440, Nov. 1981.

[65] R. K. Murthy, R. S. Sirohi, and M. P. Kothiyal, "Speckle shearing interferometry: a new method.", Applied Optics, Vol. 21, Issue 16, pp. 2865_1-2867, Aug. 1982.

[66] R. K. Mohanty, C. Joenathan, and R. S. Sirohi, "Speckle-shear interferometry with double dove prisms", Optics Communications, Vol. 47, Issue 1, pp. 27-30, Aug. 1983.

[67] D. K. Sharma, R. S. Sirohi, and M. P. Kothiyal, "Simultaneous measurement of slope and curvature with a three-aperture speckle shearing interferometer", Applied Optics, Vol. 23, Issue 10, p. 1542, May 1984.

[68] A. R. Ganesan, D. K. Sharma, and M. P. Kothiyal, "Universal digital speckle shearing interferometer.", Applied Optics, Vol. 27, Issue 22, pp. 4731-4, Nov. 1988.

[69] H. Kadono, S. Toyooka, and Y. Iwasaki, "Speckle-shearing interferometry using a liquid-crystal cell as a phase modulator", JOSA A, Vol. 8, Issue 12, p. 2001, Dec. 1991.

[70] H. A. Aebischer and S. Waldner, "Strain distributions made visible with image-shearing speckle pattern interferometry", Optics and Lasers in Engineering, Vol. 26, Issues 4-5, pp. 407-420, Mar. 1997.

[71] R. Kästle, E. Hack, and U. Sennhauser, "Multiwavelength Shearography for Quantitative Measurements of Two-dimensional Strain Distributions", Applied Optics, Vol. 38, Issue 1, p. 96, Jan. 1999.

[72] R. F. Anastasi, S. M. Serabian, R. J. Shuford, and D. K. Das-Gupta, "Nondestructive detection of simulated delaminations in composite laminates by laser-speckle shearography", Experimental Techniques, Vol. 11, Issue 6,, pp. 28-31, Jun. 1987.

[73] S. L. Toh, H. M. Shang, F. S. Chau, and C. J. Tay, "Flaw detection in composites using timeaverage shearography", Optics \& Laser Technology, Vol. 23, Issue 1, pp. 25-30, Feb. 1991.

[74] C. Shakher and A. K. Nirala, "Measurement of temperature using speckle shearing interferometry.", Applied Optics, Vol. 33, Issue 11, pp. 2125-7, Apr. 1994.

[75] B. BHADURI, M. P. KOTHIVAL, and N. KRISHNA MOHAN, "Vibration mode shape visualization with dual function DSPI system", SPIE Proceedings 6292, Interferometry XIII: Techniques and Analysis, pp. 629217.1-629217.7, .

[76] K. Habib, "Measurement of Thermal Expansion Coefficients of Thin Film of Different Organic Coatings by Shearography", KEY ENG MAT (Vol. 321 - 323), Advanced Nondestructive Evaluation I, pp. 67-70, 2006.

[77] A. Fernandez, A. Davila, C. Perez-Lopez, G. Mendiola, and J. Blanco-Garcia, "Algorithm for surface contouring using two-source phase-stepping digital shearography", SPIE Proceedings, Vol. 4419, Optical Metrology, pp. 170-173, 2001.

[78] H. Sanati, F. Reshadi, G. Faraji, N. Soltani, and E. Zalnezhad, "Evaluation of residual stress in ultrafine-grained aluminum tubes using shearography", Proceedings of the Institution of Mechanical Engineers, Part B: Journal of Engineering Manufacture, p. 0954405414534432-, Jun. 2014. 
[79] R. Krupka, T. Walz, and A. Ettemeyer, "Industrial applications of shearography for inspection of aircraft components", SPIE Proceedings, Vol. 5852, Automated NDT and Metals Evaluation, pp. 476-479, 2005.

[80] R. Růžek, R. Lohonka, and J. Jironč, "Ultrasonic C-Scan and shearography NDI techniques evaluation of impact defects identification", NDT \& E International, Vol. 39, Issue 2, pp. 132-142, Mar. 2006.

[81] W. An and K. tekniska högskolan. I. för industriell produktion, Industrial Applications of Speckle Techniques: Measurement of Deformation and Shape, p. 63, 2002.

[82] S. P. Waldner, "Quantitative strain analysis with image shearing speckle pattern interferometry (shearography)", Swiss Federal Institute of Technology Zürich, 2000.

[83] H. Helmers and M. Schellenberg, "CMOS vs. CCD sensors in speckle interferometry", Optics \& Laser Technology, Vol. 35, Issue 8, pp. 587-595, Nov. 2003.

[84] D. Knipp, H. Stiebig, and H. Wagner, "Thin film color sensors in multichannel technology", SPIE Proceedings, Vol. 4306, Novel Technologies, pp. 156-167, 2001.

[85] R. Jones and C. Wykes, Holographic and Speckle Interferometry: A Discussion of the Theory, Practice and Application of the Techniques, Cambridge University Press, p. 330, 1983.

[86] G. Zhang, Z. Wu, and Y. Li, "Speckle size of light scattered from 3D rough objects.", Optics Express, Vol. 20, Issue 4, pp. 4726-37, Mar. 2012.

[87] J. W. Goodman, "Laser Speckle and Related Phenomena", Laser Speckle and Related Phenomena Topics in Applied Physics Vol. 9, 1975.

[88] P. Hariharan, Basics of Interferometry, Elsevier, pp. 3-12, 2007.

[89] R. Sirohi, Optical Methods of Measurement: Wholefield Techniques, CRC Press, p. 328, 1999.

[90] Y. An and G. S. Schajer, "Pixel quality evaluation and correction procedures in electronic speckle pattern interferometry", Experimental Techniques, Vol. 35, Issue 5, pp. 23-29, Sep. 2011.

[91] D. C. Ghiglia and M. D. Pritt, Two-dimensional phase unwrapping: theory, algorithms, and software, Wiley, p. 493, 1998.

[92] H. Yu, S. Shao, Z. Zhang, and Q. Feng, "3D profilometry system based on absolute phase calibration", SPIE Proceedings, Vol. 6357, Signal Acquisition, Processing, and Analysis, p. 63570M-63570M-6, 2006.

[93] J.-R. Lee, D.-J. Yoon, J.-S. Kim, and A. Vautrin, "Investigation of shear distance in Michelson interferometer-based shearography for mechanical characterization", Measurement Science and Technology, Vol. 19, No 11, p. 115303, Nov. 2008.

[94] Y. Surrel and N. Fournier, "Displacement field measurement in the nanometer range", SPIE Proceedings, Vol. 2782, Grating Interferometry and Related Topics, pp. 233-242, 1996.

[95] T. W. Ng, "Shear measurement in digital speckle shearing interferometry using digital correlation", Optics Communications, Vol. 115, Issues 3-4, pp. 241-244, Mar. 1995. 
[96] R. M. Groves, S. W. James, and R. P. Tatam, "Strain measurement in curved industrial components using multicomponent shearography", SPIE Proceedings, Vol. 4398, Applications, pp. 216-224, 2001.

[97] J. Blaber, "Ncorr - 2D Digital Image Correlation Software”, Georgia Institute of Technology, 2013.

[98] U. Schnars and W. Jüptner, "Messung von Absolutverformungen mit Methoden der holografischen Interferometrie und der Elektronischen Specklemuster-Interferometrie", Laser und Optoelektronik, 24(1):59-63, 1992, pp. 59-63, 1992.

[99] H. Marwitz, "Praxis der Holografie - Grundlagen, Standard- und Spezialverfahren", Chapter 18, "Holografische Konturgenerierung, 18-2, Zwei-Wellenlängen-Methode", Expert Verlag, Stuttgart, 1990.

[100] E. Carrera, G. Giunta, and M. Petrolo, Beam Structures: Classical and Advanced Theories, John Wiley \& Sons, p. 208, 2011.

[101] G. Cloud, "Optical Methods in Experimental Mechanics. Part 27: Speckle Size Estimates", Experimental Techniques, Vol. 31, Issue 3, pp. 19-22, May 2007.

[102] R. J. Pomeroy, "The effect of anticlastic bending on the curvature of beams", International Journal of Solids and Structures, Vol. 6, Issue 2, pp. 277-285, Feb. 1970.

[103] H.-J. Kim, D.-G. Gweon, and H.-C. Kim, "Development of the integrated measuring system of strain distribution and defect using ESPI \& shearography", International Journal of Precision Engineering and Manufacturing, Vol. 13, Issue 11, pp. 1931-1939, Oct. 2012. 